\title{
MÉTODOS ALTERNATIVOS PARA REALIZAÇÃO DE TESTES DE HIPÓTESES EM DELINEAMENTOS EXPERIMENTAIS
}

\section{CRistiano NunES NESI}

Dissertação apresentada à Escola Superior de Agricultura "Luiz de Queiroz", Universidade de São Paulo, para obtenção do título de Mestre em Agronomia, Área de Concentração: Estatística e Experimentação Agronômica.

PIRACICABA

Estado de São Paulo - Brasil

Maio - 2002 


\title{
MÉTODOS ALTERNATIVOS PARA REALIZAÇÃO DE TESTES DE HIPÓTESES EM DELINEAMENTOS EXPERIMENTAIS
}

\author{
Cristiano Nunes NeSi \\ Engenheiro Agrônomo \\ Licenciado em Matemática
}

Orientador: Prof. Dr. ANTONIO AUGUSTO FRANCO GARCIA

Dissertação apresentada à Escola Superior de Agricultura "Luiz de Queiroz", Universidade de São Paulo, para obtenção do título de Mestre em Agronomia, Área de Concentração: Estatística e Experimentação Agronômica.

PIRACICABA

Estado de São Paulo - Brasil

Maio - 2002 


\title{
Dados Internacionais de Catalogação na Publicação (CIP) DIVISÃO DE BIBLIOTECA E DOCUMENTAÇÃO - ESALQ/USP
}

\author{
Nesi, C ristiano Nunes \\ Métodos a ltemativos para rea liza ção de testes de hipóteses em \\ delinea mentos experimenta is / Cristia no Nunes Nesi. - - Pirac ic aba, 2002. \\ $123 \mathrm{p}$. \\ Dissertação (mestrado) - - Escola Superior de Agricultura Luiz de \\ Queiroz, 2002. \\ Bibliografia. \\ 1. Aná lise multiva ria da 2. Delinea mento experimental 3. Esta tística 4. \\ Inferência estatístic a 5. Testes de hipóteses I. Título
}

CDD 519.56

"Permitida a cópia total ou parcial deste documento, desde que citada a fonte - $\mathrm{O}$ autor" 
Aos meus pais,

Avelino Nesi e Zilma Pereira Nunes Nesi

DEDICO

Aos meus irmãos,

Adriano, Cristiane e Adriana e ao

meu sobrinho Lucas

OFEREÇO

\section{A DEUS,}

AGRADEÇO por mais esta oportunidade. 


\section{AGRADECIMENTOS}

Aos meus pais e meus irmãos pelo amor, pela compreensão, pelo incentivo...

À Juliana Iemma, pelo carinho, pela atenção, pela convivência...

Ao Prof. Dr. Antonio Augusto Franco Garcia, pela amizade e valiosa orientação no desenvolvimento deste trabalho.

Ao Prof. Dr. Antonio Francisco Iemma, pela orientação inicial.

Aos colegas Ezequiel, Denise, Genevile, Glaucy, Ramiro, Andréia, Osmir, Mariana, Flávia e Janaína, pela convivência e amizade durante este período.

Aos amigos Jomar, Silvano, Maria Cristina e André pelas contribuições e incansável disposição em ajudar.

Aos professores do Departamento de Ciências Exatas da Escola Superior de Agricultura Luiz de Queiroz, pela transmissão dos conhecimentos, pela compreensão e amizade.

Aos funcionários do Departamento de Ciências Exatas da Escola Superior de Agricultura Luiz de Queiroz, pela atenção e dedicação.

À pesquisadora Ana Beatriz Costa Czermainski, pelo incentivo para a realização do curso.

Ao CNPq, pelo auxílio financeiro concedido para a realização do Mestrado.

A todos que, de alguma forma, contribuíram para a realização deste trabalho. 


\section{SUMÁRIO}

\begin{tabular}{|c|c|c|}
\hline & & Página \\
\hline RESUN & ……….......... & viii \\
\hline SUMM & 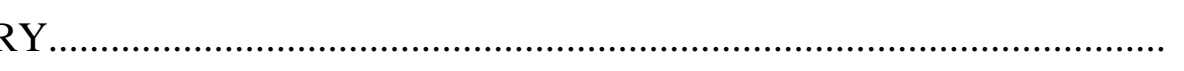 & $\mathrm{x}$ \\
\hline 1 & 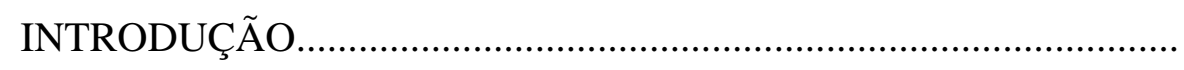 & 1 \\
\hline 2 & REVISÃO DE LITERATURA.... & 4 \\
\hline 2.1 & 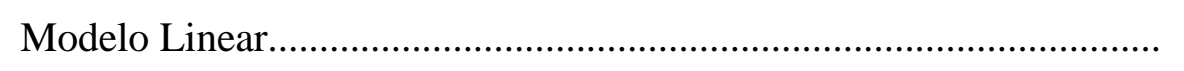 & 4 \\
\hline 2.1 .1 & Considerações iniciais......................... & 4 \\
\hline 2.1 .2 & Formas e caracterizações....... & 4 \\
\hline 2.1.2.1 & Modelo superparametrizado..... & 5 \\
\hline 2.1.2.2 & Modelo de médias de caselas..... & 6 \\
\hline 2.2 & Estimação..... & 7 \\
\hline 2.2 .1 & 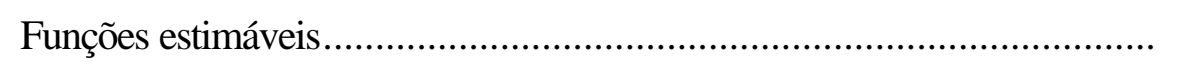 & 7 \\
\hline 2.2 .2 & Alguns aspectos da teoria geral de estimação por ponto.......................... & 8 \\
\hline 2.2 .3 & Alguns aspectos da teoria geral de estimação por intervalo.................... & 9 \\
\hline 2.3 & 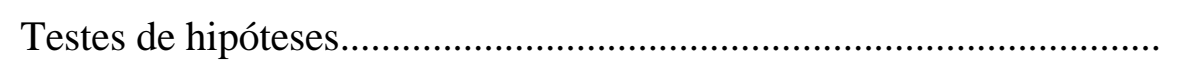 & 11 \\
\hline 2.3 .1 & Teste F.............................. & 11 \\
\hline 2.4 & Obtenção de somas de quadrados e notação R( ) ................................... & 13 \\
\hline 2.5 & Uso de restrições paramétricas para testes de hipóteses........................... & 15 \\
\hline 2.5 .1 & 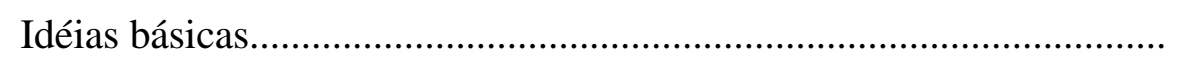 & 15 \\
\hline 2.5 .2 & Restrições estimáveis nos parâmetros e soma de quadrados do & \\
\hline & resíduo... & 15 \\
\hline 2.6 & A abordagem do SAS para testes de hipóteses................. & 21 \\
\hline 2.6 .1 & Funções estimáveis. & 21 \\
\hline
\end{tabular}


2.6.2 Tipos de hipóteses construídas pelo SAS.............................................. 23

2.6.3 Obtenção das somas de quadrados........................................................ 26

2.6.4 As funções CONTRAST e ESTIMATE................................................. 29

2.6.5 Considerações sobre o PROC GLM do SAS para análise de dados desbalanceados.............................................................................. 31

$2.7 \quad$ Variáveis auxiliares........................................................................... 32

3 MATERIAL E MÉTODOS............................................................... 34

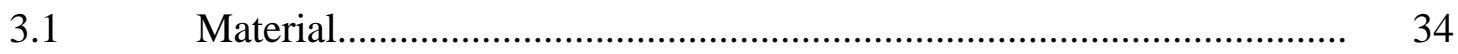

3.1.1 Conjunto A.............................................................................. 34

3.1.2 Conjunto B............................................................................. 35

3.1.3 Conjunto C................................................................................ 35

$3.2 \quad$ Métodos .......................................................................... 36

3.2.1 Modelo linear de Gauss-Markov Normal.............................................. 36

3.2.2 Análise do conjunto A....................................................................... 37

3.2.2.1 Hipóteses testadas automaticamente pelo PROC GLM do SAS........... 37

3.2.2.2 Testes de hipóteses de interesse usando contrastes................................ 38

3.2.2.3 Variáveis auxiliares............................................................................ 40

3.2.3 Análise do conjunto B ..................................................................... 43

3.2.3.1 Hipóteses testadas automaticamente pelo SAS...................................... 43

3.2.3.2 Testes de hipóteses de interesse usando contrastes................................. 44

3.2.3.3 Variáveis auxiliares......................................................................... 46

3.2.4 Análise do conjunto C....................................................................... 50

3.2.4.1 Caracterização do modelo linear.............................................................. 50

3.2.4.2 Hipóteses testadas pelo PROC GLM do SAS......................................... 51

3.2.4.2.1 Hipóteses sobre hormônios (Fator A, colunas)...................................... 52

3.2.4.2.2 Hipóteses sobre cultivares (Fator B, linhas)........................................... 53

3.2.4.2.3 Hipóteses sobre a interação.................................................................. 55

3.2.4.3 Hipóteses testadas pela função CONTRAST........................................ 56

3.2.4.4 Hipóteses testadas pela técnica da restrição paramétrica estimável...... 58 RESULTADOS E DISCUSSÃO. 


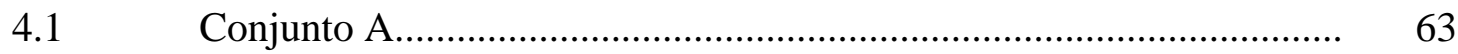

4.1.1 Hipóteses testadas automaticamente pelo PROC GLM do SAS........... 63

Desdobramento dos graus de liberdade de variedades usando

4.1.2 contrastes ortogonais......................................................................... 66

4.1.3 Análise de variância pela técnica das variáveis auxiliares...................... 68

$4.2 \quad$ Conjunto B................................................................................ 73

4.2.1 Hipóteses testadas automaticamente pelo PROC GLM do SAS........... 73

4.2.2 Desdobramento dos graus de liberdade de variedades usando contrastes ortogonais....................................................................... 75

4.2.3 Análise de variância pela técnica das variáveis auxiliares...................... 78

$4.3 \quad$ Conjunto C ................................................................................ 84

4.3.1 Hipóteses testadas automaticamente pelo PROC GLM do SAS........... 84

4.3.2 Testes de hipóteses usando a função CONTRAST................................. 89

4.3.3 Testes de hipóteses pela técnica da restrição paramétrica estimável..... 92

$4.4 \quad$ Considerações gerais............................................................................ 104

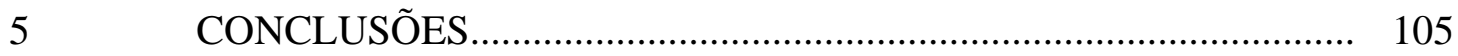

REFERÊNCIAS BIBLIOGRÁFICAS............................................................... 106

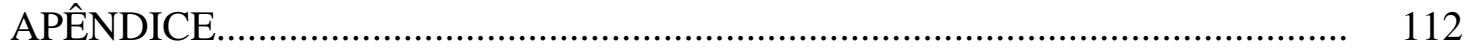




\title{
MÉTODOS ALTERNATIVOS PARA REALIZAÇÃO DE TESTES DE HIPÓTESES EM DELINEAMENTOS EXPERIMENTAIS
}

\author{
Autor: CRISTIANO NUNES NESI
}

Orientador: Prof. Dr. ANTONIO AUGUSTO FRANCO GARCIA

\section{RESUMO}

Na estatística experimental, especificamente quando se faz análise de variância, os testes de hipóteses têm sido amplamente utilizados para se concluir a respeito das fontes de variação consideradas nos modelos lineares. Para tanto, é comum a utilização de sistemas estatísticos que fornecem análises de variância e a estatística $F$, entre outras, para a tomada de decisões. Entretanto, o teste $F$ numa análise de variância para tratamentos com mais de um grau de liberdade proporciona informações gerais, relacionadas com o comportamento médio dos tratamentos. Por essa razão, deve-se planejar comparações objetivas, fazendo-se desdobramentos dos graus de liberdade de tratamentos para obter informações mais específicas. Nesse sentido, uma técnica usada para esses desdobramentos baseia-se na utilização de contrastes, sendo necessário que cada componente seja explicado por um contraste, com todos os contrastes sendo ortogonais entre si, para que as comparações sejam independentes. Entretanto, essa técnica torna-se complexa à medida que o número de tratamentos aumenta. Frente a isso, utilizando-se os dados provenientes de um experimento de competição entre dois grupos de variedades de cana-de-açúcar, inteiramente ao acaso com seis tratamentos e cinco repetições, e também nos dados obtidos de um experimento fictício de competição entre híbridos de milho no delineamento blocos casualizados, propôs-se uma técnica, 
empregando variáveis auxiliares, para facilitar o desdobramento ortogonal dos graus de liberdade de tratamentos, procurando-se evidenciar que essa técnica facilita o desdobramento ortogonal dos graus de liberdade de tratamentos e tem resultados equivalentes aos obtidos utilizando-se a função CONTRAST do PROC GLM do SAS. Outro problema refere-se à análise de experimentos fatoriais com desbalanceamento das amostras, tendo em vista que as técnicas de estimação de parcelas perdidas não resolvem satisfatoriamente o problema, principalmente se existem muitas parcelas perdidas. Quando os dados são desbalanceados, há necessidade de se conhecer que hipóteses estão sendo testadas e se estas são de interesse do pesquisador, devido à complexidade dessas hipóteses, principalmente em presença de caselas vazias. Além disso, muito têm sido escrito sobre os diferentes resultados da análise de variância apresentados por sistemas estatísticos para dados desbalanceados com caselas vazias, o que tem gerado confusão entre os pesquisadores. Com a finalidade de propor um método alternativo para a obtenção de hipóteses de interesse, utilizaram-se os resultados de um experimento fatorial 2x3, inteiramente ao acaso, com quatro repetições, para testar os efeitos de três reguladores de crescimento (hormônios), sobre a propagação "in vitro" de dois portaenxertos (cultivares) de macieira. Assim, diante do fato que testar uma hipótese é equivalente a impor uma restrição estimável aos parâmetros do modelo, utilizaram-se restrições paramétricas estimáveis como um critério alternativo para realizar testes de hipóteses de interesse em modelos lineares com dados desbalanceados. Os resultados mostram que esse método permite que o pesquisador teste diretamente hipóteses de seu interesse, com resultados equivalentes aos encontrados com a função CONTRAST do PROC GLM do SAS. 


\title{
ALTERNATIVE METHODS FOR TESTING HYPOTHESES IN EXPERIMENTAL DESIGNS
}

\author{
Author: CRISTIANO NUNES NESI \\ Adviser: Prof. Dr. ANTONIO AUGUSTO FRANCO GARCIA
}

\section{SUMMARY}

For experimental designs, it is usually necessary to do tests of hypotheses to conclude about effects considered in the linear models. In these cases, it is common to use statistical softwares that supply the analyses of variance and F statistics, among others, for taking decisions. However, the test $\mathrm{F}$ in an analysis of variance for sources of variation with more than a degree of freedom provides general information, about significant differences of levels of the factor. Therefore, it should be planned objective comparisons, making orthogonal decompositions of the degrees of the effects of interest to get more specific information. One technique used frequently based on the orthogonal contrasts, so that the comparisons are independent. However, this technique becomes complex as the number of levels of the factor increases. To study alternative methods to do these comparisons, we use data from a yield trail experiment considering two groups of varieties of sugarcane, in a complete randomized design with 6 treatments and 5 repetitions. Also, we use data from a fictitious experiment comparing hybrids of maize in the randomized complete block design. The technique of analysis using dummy variables to facilitate the orthogonal decomposition of degrees of freedom of treatments was proposed. This technique facilitates the orthogonal decomposition and has the same results of those obtained the function CONTRAST of PROC GLM of SAS. Another 
situation considered involves experiments with unbalanced data. In this case, it is possible to suppose that there is the necessity of knowing what hypotheses are being tested and if they are useful. Much has been written on the different results of analysis of variance presented by statistical software for unbalanced data. This can create confusion to the researcher. To illustrate, we used the results of an $2 \times 3$ factorial experiment with 4 replicates, to test the effect of 3 hormones, on the propagation of 2 in vitro cultivars of apple trees. Thus, considering that to test a hypotheses is equivalent to impose an estimable restriction to the parameters of the model, we use these restrictions as an alternative criteria to directly carry out tests of hypotheses in linear models with unbalanced data. The results showed that this procedure is equivalent of that used by the function CONTRAST of PROC GLM/SAS. 


\section{INTRODUÇÃO}

$\mathrm{Na}$ estatística experimental, especificamente quando se faz análise de variância, os testes de hipóteses têm sido amplamente utilizados para se concluir a respeito das fontes de variação consideradas nos modelos lineares. Para tanto, é comum a utilização de sistemas estatísticos que fornecem análises de variância e a estatística $F$, entre outras, para a tomada de decisões.

Banzatto \& Kronka (1995) citam que, quando se aplica o teste $F$ numa análise de variância para tratamentos com mais de um grau de liberdade, pode-se obter apenas informações gerais, relacionadas com o comportamento médio dos tratamentos, pois o teste representa um teste médio de diversas comparações independentes. Então, se apenas uma das comparações envolve uma diferença marcante, um teste $F$ médio pode falhar para evidenciar a diferença existente. Por essa razão, deve-se planejar comparações objetivas, fazendo-se desdobramentos dos graus de liberdade de tratamentos para obter informações mais específicas, relacionadas com o comportamento médio de cada um dos componentes do desdobramento.

Após a decomposição ortogonal dos graus de liberdade, pode-se aplicar o teste $F$ a cada um dos componentes do desdobramento, sendo que para I tratamentos pode-se estabelecer $(I-1)$ comparações independentes. Uma técnica muito usada baseia-se na utilização de contrastes, sendo necessário que cada componente seja explicado por um contraste, com todos os contrastes sendo ortogonais entre si, para que as comparações sejam independentes. Entretanto, a utilização de contrastes torna-se complexa à medida que o número de tratamentos aumenta, como ocorre por exemplo nos programas de melhoramento genético vegetal, em que é comum o pesquisador planejar um experimento com um número relativamente grande de tratamentos a serem comparados. 
Outro problema em análise de variância refere-se à análise de experimentos em que houve perda de parcelas, o que leva ao desbalanceamento das amostras. Iemma (1995) cita que as técnicas de estimação de parcelas perdidas, apesar de largamente abordadas na literatura, não resolveram satisfatoriamente o problema, principalmente se existem muitas parcelas perdidas.

A análise de um conjunto de dados balanceado, ou seja, em que cada casela contém um mesmo número de observações (Searle 1987), é relativamente fácil, bem conhecida e, de acordo com Speed et al. (1978), há um consenso sobre a realização de análise de variância e testes de hipóteses. Entretanto, quando os dados são desbalanceados, ou seja, o número de observações por casela é diferente (Searle, 1987), há necessidade de se conhecer que hipóteses estão sendo testadas e se estas são de interesse do pesquisador (Mondardo, 1994). Muitos pesquisadores têm demonstrado preocupação com a complexidade dessas hipóteses, principalmente em presença de caselas vazias (por exemplo, Searle, 1971; Santos, 1994; Camarinha Filho, 1995). Além disso, muito têm sido escrito sobre os diferentes resultados da análise de variância apresentados por sistemas estatísticos para dados desbalanceados com caselas vazias, o que segundo Hocking \& Speed (1975), Speed et al. (1978), Searle (1987), tem gerado confusão entre os pesquisadores.

Segundo Iemma (1993), a ocorrência de caselas vazias pode conduzir a alguns problemas em relação à estimação de funções paramétricas afetando a interpretação prática dos resultados, no sentido de que as hipóteses de interesse do pesquisador, que em geral são aquelas que envolvem funções de um mesmo parâmetro, podem não ser testáveis. Esses problemas refletem-se sobre a interpretação das verdadeiras hipóteses testadas pelas somas de quadrados. Pode-se citar alguns problemas, como por exemplo, a desconexão, ou seja, a impossibilidade de se estimar certas combinações lineares dos parâmetros do modelo correspondente (Searle, 1987), o teste da interação, que geralmente testa que somente algumas interações são nulas, sendo que o número de graus de liberdade da interação deve ser escolhido com cautela. Além disso, as hipóteses sobre médias não ponderadas de linhas e colunas, em geral, têm uma interpretação 
diferente daquelas com todas as caselas ocupadas além do fato de que a interpretação das hipóteses depende da posição e do número de caselas vazias.

Diante do exposto, este trabalho tem como objetivos:

i) Propor o uso de uma técnica, empregando variáveis auxiliares, para facilitar o desdobramento ortogonal dos graus de liberdade de tratamentos;

ii) Evidenciar as vantagens dessa técnica e sua equivalência com a função CONTRAST do PROC GLM do SAS, para o desdobramento ortogonal dos graus de liberdade de tratamentos;

iii) Propor o uso de restrições paramétricas estimáveis para desenvolver um critério alternativo para realizar testes de hipóteses de interesse em modelos lineares com dados desbalanceados, permitindo que o pesquisador teste diretamente hipóteses de seu interesse;

iv) Discutir as equivalências entre a função CONTRAST do PROC GLM do SAS e o critério baseado no emprego de restrição paramétrica estimável para realização de testes de hipóteses de interesse em experimentos desbalanceados. 


\section{REVISÃO DE LITERATURA}

\subsection{Modelo Linear}

\subsubsection{Considerações iniciais}

Uma vez que será amplamente estudado no presente trabalho, será apresentada a seguir a descrição de alguns aspectos da teoria de modelos lineares. Conforme Rao (1945), o modelo linear de Gauss-Markov pode ser escrito como

$$
y=X \boldsymbol{\theta}+e,
$$

em que:

$y$ : vetor de realizações da variável aleatória $Y$;

$X$ : matriz conhecida de incidência dos efeitos fixos (matriz de constantes que identifica o delineamento experimental);

$\boldsymbol{\theta}$ : vetor de parâmetros desconhecidos;

$e$ : vetor de variáveis aleatórias não observáveis tal que $e \sim N\left(\phi, I \sigma^{2}\right)$.

\subsubsection{Formas e caracterizações}

Segundo Speed et al. (1978), Searle (1987) e Iemma (1991), entre outros, o modelo linear (1) pode ser apresentado, de acordo com o interesse do pesquisador, sob diferentes formas e caracterizações. Dentre várias outras formas descritas na literatura, destacam-se o modelo superparametrizado (modelo-S) e o modelo de médias de caselas (modelo-M). Segundo Camarinha Filho (1995), embora os dois modelos sejam 
estatisticamente equivalentes, no momento da construção e interpretação das hipóteses ambos são fundamentais, pois o modelo-M facilita a construção das hipóteses e o modelo-S deixa claro quais são os efeitos do modelo linear envolvidos na referida hipótese. O modelo-S é utilizado pelo PROC GLM do SAS, tanto na apresentação de suas funções estimáveis quanto para as estimativas de cada um dos parâmetros do modelo linear. Por fazerem parte do presente trabalho, serão detalhados a seguir.

\subsubsection{Modelo superparametrizado}

Segundo Searle (1971), um modelo linear superparametrizado exibe, para cada efeito dos fatores envolvidos, seu respectivo parâmetro. Este tipo de modelo será denotado aqui por

$$
y=X \boldsymbol{\theta}+e,
$$

com significados já descritos. Essa notação se faz necessária para diferenciá-lo do modelo de médias de caselas, a ser apresentado oportunamente.

De acordo com o interesse do pesquisador, o modelo-S pode assumir diferentes caracterizações, por meio de parametrizações sucessivas. Assim, como exemplo, partindo-se do modelo mais simples com uma constante até um modelo com dois fatores cruzados, tem-se:

$$
\begin{array}{llll}
y=X_{1} \boldsymbol{\theta}_{1}+e_{1} & \Rightarrow & y_{i}=\mu+e_{i} \\
y=X_{2} \theta_{2}+e_{2} & \Rightarrow & y_{i k}=\mu+\alpha_{i}+e_{i k} \\
y=X_{3} \theta_{3}+e_{3} & \Rightarrow & y_{j k}=\mu+\beta_{j}+e_{j k} \\
y=X_{4} \theta_{4}+e_{4} & \Rightarrow & y_{i j k}=\mu+\alpha_{i}+\beta_{j}+e_{i j k} \\
y=X \boldsymbol{\theta}+e & \Rightarrow & y_{i j k}=\mu+\alpha_{i}+\beta_{j}+\gamma_{i j}+e_{i j k}
\end{array}
$$

em que:

$i=1, \ldots, a$ linhas;

$j=1, \ldots, b$ colunas; 
$k$ : repetições;

$X_{1}$ : primeira coluna da matriz $X$;

$\theta_{1}$ : vetor do parâmetro $\mu$;

$\mu$ : constante inerente a todas as observações;

$X_{2}$ : matriz formada pelas colunas da matriz $X$ referentes a $\mu$ e $\alpha_{i}$;

$\boldsymbol{\theta}_{2}$ : vetor formado pelos parâmetros $\mu$ e $\alpha_{i}$;

$\alpha_{i}$ : efeito do $i$-ésimo nível do fator $A$;

$X_{3}$ : matriz formada pelas colunas da matriz $X$ referentes a $\mu$ e $\beta_{j}$;

$\theta_{3}$ : vetor formado pelos parâmetros $\mu$ e $\beta_{j}$;

$\beta_{j}$ : efeito do $j$-ésimo nível do fator $B$;

$X_{4}$ : matriz formada pelas colunas da matriz $X$ referentes a $\mu, \alpha_{i}$ e $\beta_{j}$;

$\boldsymbol{\theta}_{4}$ : vetor formado pelos parâmetros $\mu, \alpha_{i}$ e $\beta_{j}$;

$X$ : matriz do delineamento;

$\boldsymbol{\theta}$ : vetor de parâmetros para o modelo completo;

$y_{i j k}$ : observação resultante do $i$-ésimo nível do fator $A, j$-ésimo nível do fator $B$ e $k$-ésima repetição;

$\gamma_{i j}$ : efeito da interação entre o $i$-ésimo nível do fator $A$ e o $j$-ésimo nível do fator $B$;

$e_{i j k}$ : erro aleatório referente ao $i$-ésimo nível do fator $A, j$-ésimo nível do fator $B$ e $k$-ésima repetição.

Observa-se que de (2.1) a (2.5) existe uma parametrização sucessiva e ordenada.

\subsubsection{Modelo de médias de caselas}

O modelo de médias de caselas apresenta a vantagem de descrever hipóteses com grande simplicidade, em relação ao modelo-S. Define-se o modelo-M, de acordo com Elston \& Bush (1964) e Speed et al. (1978), como o modelo linear:

$$
y=W \mu+e
$$


em que:

$y$ : vetor de realizações da variável aleatória $Y$;

$W$ : matriz do delineamento, conhecida;

$\mu$ : vetor de médias das populações das quais são retiradas as amostras aleatórias que compõem as caselas;

$e$ : vetor de variáveis aleatórias não observáveis tal que $e \sim N\left(\phi, I \sigma^{2}\right)$.

Em correspondência com o modelo-S tem-se:

$$
\begin{array}{lll}
y_{i}=\mu+e_{i} & \Rightarrow & y_{i}=\mu+e_{i} \\
y_{i k}=\mu+\alpha_{i}+e_{i k} & \Rightarrow & y_{i k}=\mu_{i}+e_{i k} \\
y_{j k}=\mu+\beta_{j}+e_{j k} & \Rightarrow & y_{j k}=\mu_{j}+e_{j k} \\
y_{i j k}=\mu+\alpha_{i}+\beta_{j}+e_{i j k} & \Rightarrow & y_{i j k}=\mu_{i}+\beta_{j}+e_{i j k} \\
y_{i j k}=\mu+\alpha_{i}+\beta_{j}+\gamma_{i j}+e_{i j k} & \Rightarrow & y_{i j k}=\mu_{i j}+e_{i j k}
\end{array}
$$

em que $\mu_{i}=\mu+\alpha_{i}, \mu_{j}=\mu+\beta_{j}$ e $\mu_{i j}=\mu+\alpha_{i}+\beta_{j}+\gamma_{i j}$.

Os significados são os mesmos que aqueles apresentados em 2.1.2.1.

\subsection{Estimação}

\subsubsection{Funções estimáveis}

De acordo com Scheffé (1961), uma função paramétrica é definida como sendo uma função linear dos parâmetros desconhecidos $\left\{\boldsymbol{\theta}_{1}, \ldots, \boldsymbol{\theta}_{n}\right\}$, com coeficientes constantes e conhecidos $\left\{\lambda_{1}, \ldots, \lambda_{n}\right\}$. Segundo Rao (1945), uma função linear paramétrica $\lambda^{\prime} \boldsymbol{\theta}$ é dita função estimável no modelo $y=X \boldsymbol{\theta}+e$, se e somente se existe uma combinação linear das observações $a^{\prime} y$, tal que $E\left[a^{\prime} y\right]=\lambda^{\prime} \theta$. Se não existe $a$ tal que a igualdade se verifique, $\lambda^{\prime} \theta$ é dita não estimável nesse modelo. 
Essa definição de estimabilidade é de reconhecida importância em demonstrações teóricas, porém em aspectos práticos ela é pouco operacional (Iemma, 1987). Frente a isto, foram estabelecidas regras práticas de estimabilidade, entre as quais estão as funções básicas estimáveis. Isso é feito observando se $\lambda^{\prime}$ é uma combinação das linhas da matriz $X$; caso isto ocorra, $\lambda^{\prime} \theta$ é uma função estimável (Scheffé, 1961).

Dessa forma, segundo Searle (1987), o estimador de $\lambda^{\prime} \theta$ é $\lambda^{\prime} \theta^{0}=\lambda^{\prime} \theta$, que é invariante para todo e qualquer $\theta^{0}=\left(X^{\prime} X\right)^{G} X^{\prime} y$ solução das equações normais, com $E\left[\lambda^{\prime} \boldsymbol{\theta}^{0}\right]=\lambda^{\prime} \boldsymbol{\theta}$ e $\operatorname{var}\left[\lambda^{\prime} \boldsymbol{\theta}^{0}\right]=\lambda^{\prime}\left(X^{\prime} X\right)^{G} \lambda \sigma^{2}$, sendo $\left(X^{\prime} X\right)^{G}$ uma inversa generalizada qualquer de $X^{\prime} X$ e $\sigma^{2}$ a variância da população. Nesse contexto, o BLUE ("Best Linear Unbiased Estimator") de $\lambda^{\prime} \boldsymbol{\theta}$, como em Scheffé (1961), é obtido de modo único por $\lambda^{\prime} \boldsymbol{\theta}=\lambda^{\prime} \boldsymbol{\theta}^{0}$. Assim, é possível construir uma regra prática para obtenção dos BLUE's de interesse.

\subsubsection{Alguns aspectos da teoria geral de estimação por ponto}

A seguir, serão apresentados alguns conceitos teóricos, considerando-se que há interesse em estimar os elementos de $\boldsymbol{\theta}$ ou uma combinação linear deles. Para tanto, será considerado o modelo-S.

Segundo Scheffé (1961) e Searle (1987), quando a matriz $X$ do delineamento é de posto coluna completo, ou seja, $r\left[{ }_{n} X_{p}\right]=$ posto da matriz $X=p$, exceto devido a colinearidades, $X^{\prime} X$ é positiva definida e, portanto, não singular. Assim, o sistema de equações normais tem solução única dada por $\hat{\theta}=\left(X^{\prime} X\right)^{-1} X^{\prime} y$ (Searle, 1971). Além disso, $\hat{\boldsymbol{\theta}}$ é estimador não viesado para $\boldsymbol{\theta}$, pois (Searle, 1971; Hocking, 1985; Iemma, 1989):

$$
E(\hat{\boldsymbol{\theta}})=\left(X^{\prime} X\right)^{-1} X^{\prime} E(y)=\left(X^{\prime} X\right)^{-1} X^{\prime} X \boldsymbol{\theta}=\boldsymbol{\theta}
$$

Nesse caso, a solução de mínimos quadrados (“Least Square Solution” - L.S.S.) coincide com o estimador de mínimos quadrados ordinários para $\boldsymbol{\theta}$ (“Ordinary Least 
Square Estimator" - O.L.S.E.), cujos componentes do vetor $\boldsymbol{\theta}$ (cada parâmetro do modelo) são estimáveis.

De acordo com Searle (1987), ocorre geralmente em delineamentos experimentais que $X$ não tem posto coluna completo, ou seja, $r\left[{ }_{n} X_{p}\right]<\operatorname{mín}\{n, p\}$. Então, $X^{\prime} X$ é singular e o sistema de equações normais, sempre consistente, é indeterminado. Suas soluções podem ser obtidas por $\theta^{0}=\left(X^{\prime} X\right)^{G} X^{\prime} y$, que também são soluções de mínimos quadrados para o sistema $y=X \Theta$ inconsistente. Agora $\theta^{0}$ não é estimador não viesado para $\boldsymbol{\theta}$, pois (Iemma, 1989):

$$
E\left[\boldsymbol{\theta}^{0}\right]=\left(X^{\prime} X\right)^{G} X^{\prime} E[y]=\left(X^{\prime} X\right)^{G} X^{\prime} X \boldsymbol{\theta} .
$$

Em (5), definindo-se $H=\left(X^{\prime} X\right)^{G} X^{\prime} X$, tem-se que $E\left[\theta^{0}\right]=H \boldsymbol{\theta}$. Desta forma, são estimáveis as funções paramétricas de $\boldsymbol{\theta}$ que estão em $H \boldsymbol{\theta}$, seguindo o que foi apresentado por Scheffé (1961). Segundo Iemma (1987), $H$ varia em função da inversa generalizada de $X^{\prime} X$, resultando em diferentes conjuntos de funções estimáveis. Em suma, são estimáveis apenas algumas combinações lineares de $\boldsymbol{\theta}$.

\subsubsection{Alguns aspectos da teoria geral de estimação por intervalo}

De acordo com Graybill (1961), na obtenção de estimativas por ponto, o procedimento consiste em escolher uma função na amostra aleatória que seja a melhor estimativa do parâmetro desejado. No contexto de modelos lineares aplicados a delineamentos experimentais, essas funções são obtidas em $\boldsymbol{\theta}^{0}$, como descrito. Em situações reais, geralmente não é suficiente obter apenas uma estimativa do parâmetro sob investigação, mas deve-se também ter alguma confiança de que o valor obtido está entre limites conhecidos.

Nesse contexto, tem-se interesse em obter também estimativas por intervalo, ou intervalos de confiança para funções estimáveis, e estimativas por região ou regiões de confiança para um conjunto de funções estimáveis. 
Scheffé (1961) define região de confiança (RC) para um conjunto $B^{\prime} \boldsymbol{\theta}$ de funções estimáveis, linearmente independentes, ao nível de confiança $1-\alpha$, como a região delimitada pelo elipsóide dado por:

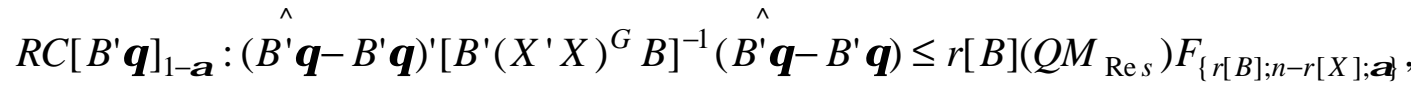

em que

$B$ : matriz dos coeficientes da função paramétrica estimável $B^{\prime} \boldsymbol{\theta}$;

$\left(X^{\prime} X\right)^{G}$ : inversa generalizada qualquer de $\left(X^{\prime} X\right)$;

$r[B]:$ posto da matriz $B$;

$\boldsymbol{\theta}$ : vetor de parâmetros desconhecidos;

$Q M_{\operatorname{Re} s}:$ quadrado médio do resíduo;

$F$ : valor da distribuição $\mathrm{F}$, tabelado em função de $r[B]$ e de $n-r[X]$ graus de liberdade e do coeficiente de confiança $\alpha$;

$n$ : número de observações;

$r[X]$ : posto da matriz $X$.

Assim, para a hipótese $H_{0}: B^{\prime} \boldsymbol{\theta}=\boldsymbol{\phi}$, rejeita-se $H_{0}$ ao nível $\alpha$ de significância, se o elipsóide construído ao nível de confiança conjunto $1-\alpha$ não contém a origem dos eixos (ponto $\phi)$, ou seja:

$$
\left(\hat{B^{\prime} \boldsymbol{\theta}}\right)^{\prime}\left[B^{\prime}\left(X^{\prime} X\right)^{G} B\right]^{-1}\left(\hat{\left.B^{\prime} \boldsymbol{\theta}\right)}>r[B]\left(Q M_{\operatorname{Re} s}\right) F_{\{r[B] ; n-r[X] ; \alpha\}} \cdot\right.
$$

Um intervalo de confiança (IC) é um caso especial de região de confiança unidimensional (Scheffé, 1961). Assim, dada a simetria da distribuição $t$ de Student, uma regra para construir estimativas por intervalo para uma única função linear paramétrica $\lambda^{\prime} \theta$ estimável ao nível de confiança 1- $\alpha$, é dada por (Scheffé, 1961; Searle, 1987):

$$
I C\left[\lambda^{\prime} \theta\right]_{1-\alpha}: \lambda^{\prime} \theta \pm t_{\{n-r[X] ; \alpha}, \sqrt{\left.\hat{\operatorname{var}\left[\lambda^{\prime} \theta\right.}\right]}
$$

em que: 
$t_{\{n-r[X] ; \mathscr{Q} / 2\}}$ : valor da distribuição $t$, tabelado em função de $n-r[X]$ graus de liberdade e do coeficiente de confiança $\alpha / 2$;

$\hat{\operatorname{var}}\left[\lambda^{\prime} \theta\right]$ : estimativa da variância da estimativa de $\lambda^{\prime} \theta$.

Dessa forma, rejeita-se $H_{0}: \lambda^{\prime} \theta=\phi$, ao nível $\alpha$ de significância, se a estimativa por intervalo, construída ao nível de significância $1-\alpha$, não contém o ponto zero.

\subsection{Testes de hipóteses}

Uma vez apresentados alguns aspectos sobre estimação por ponto e por intervalo, serão agora apresentados conceitos sobre testes de hipóteses, no contexto dos modelos lineares.

\subsubsection{Teste $\mathbf{F}$}

Hocking (1985) cita que a distribuição de probabilidades do estimador proporciona informações para se fazer inferências sobre o parâmetro desconhecido $\boldsymbol{\theta}$. Isso pode ser usado pelo pesquisador de duas diferentes maneiras para interpretar seus resultados: construção de regiões ou intervalos de confiança para funções lineares de $\boldsymbol{\theta}$ ou, alternativamente, construir testes de hipóteses a respeito de funções de $\boldsymbol{\theta}$.

De acordo com Searle (1987), uma hipótese linear geral associada ao conjunto de parâmetros $\boldsymbol{\theta}$ pode ser definida sob a forma $H_{0}: B^{\prime} \boldsymbol{\theta}=c$, em que $B^{\prime}$ é uma matriz conhecida (reflete as funções lineares de $\boldsymbol{\theta}$ que se deseja testar) e $c$ é um vetor conhecido, geralmente nulo, que reflete a diferença presente no contraste criado em $B^{\prime}$. Quando $B^{\prime}$ tem posto linha completo e os elementos da função paramétrica $B^{\prime} \theta$ são estimáveis, a hipótese $H_{0}: B^{\prime} \boldsymbol{\theta}=c$ é dita hipótese testável. Isto posto, pode-se calcular, por exemplo, uma estatística $F$ conveniente.

Para tanto, pode-se considerar a seguinte forma quadrática (Searle, 1971)

$$
Q=\left(B^{\prime} \boldsymbol{\theta}^{0}-c\right)^{\prime}\left[B^{\prime}\left(X^{\prime} X\right)^{G} B\right]^{-1}\left(B^{\prime} \boldsymbol{\theta}^{0}-c\right),
$$


tal que

$$
\frac{Q}{\sigma^{2}} \sim \chi_{\left[r[B] ; \frac{\left(B^{\prime} \boldsymbol{\theta}^{0}-c\right) !\left[B^{\prime}\left(X^{\prime} X\right)^{G} B\right]^{-1}\left(B^{\prime} \boldsymbol{\theta}^{0}-c\right)}{2 \sigma^{2}}\right]},
$$

em que

$\chi^{\prime 2}$ : distribuição de qui-quadrado não central, com $r[B]$ graus de liberdade e parâmetro de não centralidade $\left(\frac{\left(B^{\prime} \Theta^{0}-c\right)^{\prime}\left[B^{\prime}\left(X^{\prime} X\right)^{G} B\right]^{-1}\left(B^{\prime} \Theta^{0}-c\right)}{2 \sigma^{2}}\right)$. Os demais termos têm os mesmos significados já descritos.

Sabe-se que $Q$ e $S Q_{\operatorname{Res}}$ (soma de quadrados do resíduo) são independentemente distribuídas (Iemma, 1989). Então (Searle, 1971),

$$
F\left(H_{0}\right)=\frac{\frac{Q}{r[B]}}{\frac{S Q_{\operatorname{Re} s}}{n-r[X]}}=\frac{Q}{r[B] \hat{\sigma}^{2}} \sim F^{\prime}\left\{r[B], n-r[X], \frac{\left(B^{\prime} \Theta^{0}-c\right)^{\prime}\left[B^{\prime}\left(X^{\prime} X\right)^{G} B\right]^{-1}\left(B^{\prime} \Theta^{0}-c\right)}{2 \sigma^{2}}\right\},
$$

em que

$F\left(H_{0}\right)$ : estatística $F$ para a hipótese $H_{0}$;

$n:$ número de observações;

$\hat{\sigma}^{2}$ : estimativa da variância populacional;

$F^{\prime}$ : distribuição de $\mathrm{F}$ não central, com $r[B]$ e $n-r[X]$ graus de liberdade e parâmetro de não centralidade $\frac{\left(B^{\prime} \boldsymbol{\Theta}^{0}-c\right)^{\prime}\left[B^{\prime}\left(X^{\prime} X\right)^{G} B\right]^{-1}\left(B^{\prime} \boldsymbol{\Theta}^{0}-c\right)}{2 \sigma^{2}}$ (significados já apresentados).

Assim, a estatística $F$ para testar uma hipótese linear geral $H_{0}: B^{\prime} \boldsymbol{\theta}=c$, é (Iemma, 1989):

$$
F\left(H_{0}\right)=\frac{Q}{r[B] \hat{\sigma}^{2}}=\frac{\left(B^{\prime} \boldsymbol{\theta}^{0}-c\right)^{\prime}\left[B^{\prime}\left(X^{\prime} X\right)^{G} B\right]^{-1}\left(B^{\prime} \boldsymbol{\theta}^{0}-c\right)}{r[B] \hat{\sigma}^{2}} .
$$

Geralmente, principalmente em análises de variância, tem-se que $c$ é nulo. Então, com a hipótese na forma $H_{0}: B^{\prime} \Theta=0$, a estatística $F$ adequada para testá-la é: 


$$
F\left(H_{0}\right)=\frac{Q}{r[B] \hat{\sigma}^{2}}=\frac{\left(B^{\prime} \boldsymbol{\theta}^{0}\right)^{\prime}\left[B^{\prime}\left(X^{\prime} X\right)^{G} B\right]^{-1}\left(B^{\prime} \boldsymbol{\theta}^{0}\right)}{r[B] \hat{\sigma}^{2}} .
$$

\subsection{Obtenção de somas de quadrados e notação $R($ )}

A idéia de utilização da notação $R($ ), consagrada por Searle (1971), está ligada ao método de ajuste de constantes, descrito por Yates (1934), e consiste na redução na soma de quadrados após o ajuste de diferentes modelos.

Para um modelo de classificação simples, como apresentado em (2.2), a diferença

$$
R(\mu, \alpha)-R(\mu)
$$

é definida como a redução na soma de quadrados devido ao ajuste de dois modelos diferentes, um contendo $\mu$ e um fator $\alpha$ e outro contendo apenas $\mu$. Em outras palavras, tendo-se ajustado $\mu$, a diferença (10) representa a redução na soma de quadrados devido ao ajuste de um fator $\alpha$ adicionalmente a $\mu$. Isso é representado da seguinte forma:

$$
R(\alpha \mid \mu)=R(\mu, \alpha)-R(\mu) .
$$

Esse procedimento é análogo para outros modelos. Por exemplo, para um modelo de classificação dupla,

$$
R(\alpha \mid \mu, \beta)=R(\mu, \alpha, \beta)-R(\mu, \beta)
$$

é a redução na soma de quadrados devido ao ajuste de $\alpha$ após $\mu$ e $\beta$.

De acordo com Iemma \& Perri (1997), as somas de quadrados associadas aos modelos (2) e (3) podem ser convenientemente descritas por meio da notação $R($ ), sobretudo para o modelo-S, fazendo-se parametrizações sucessivas e subtrações adequadas.

Usando-se essa notação e particionando-se convenientemente a matriz do delineamento e o vetor de parâmetros, pode-se associar a cada modelo, do mais simples ao mais completo, uma soma de quadrados de parâmetros. Por exemplo, pode-se tomar 
$X=\left[\begin{array}{l:l:l:l}X_{1} & X_{2} & X_{3} & X_{23}\end{array}\right] ; \quad \boldsymbol{\theta}=\left[\begin{array}{c}\boldsymbol{\theta}_{1} \\ -\boldsymbol{\theta}_{2} \\ \hdashline \boldsymbol{\theta}_{3} \\ \hdashline \boldsymbol{\theta}_{23}\end{array}\right]$.

Se considerarmos o modelo $y_{i}=\mu+e_{i}$ (matricialmente, $y=X_{1} \theta_{1}+e_{1} \mathrm{e}$

$\left.\theta_{1}{ }^{\prime}=[\mu]\right)$, obtém-se segundo Iemma \& Perri (1997), entre outros:

$R(\mu)=\theta_{1}^{0 \prime} X_{1}^{\prime} y=$ correção $=S . Q \cdot$ Parâmetros do modelo considerando $\mu$.

Para o modelo $y_{i}=\mu+\alpha_{i}+e_{i j}$ (matricialmente, $y=X_{2} \theta_{2}+e_{2}$ e $\theta_{1}{ }^{\prime}=[\mu, \alpha]$ ), tem-se:

$R(\mu, \boldsymbol{\alpha})=\boldsymbol{\theta}_{2}^{0 \prime} X_{2}{ }^{\prime} y=S . Q \cdot$ Parâmetros do modelo considerando $\mu$ e $\boldsymbol{\alpha}$.

Então,

$$
R(\alpha \mid \mu)=R(\mu, \alpha)-R(\mu)
$$

De modo análogo, obtém-se:

$R(\mu, \beta)=\theta_{3}^{0 \prime} X_{3}^{\prime} y=S . Q$. Parâmetros do modelo considerando $\mu$ e $\beta$, com

$$
R(\beta \mid \mu)=R(\mu, \beta)-R(\mu) .
$$

Ainda:

$R(\mu, \alpha, \beta)=\theta_{4}^{0 \prime} X_{4}^{\prime} y=S . Q$. Parâmetros do modelo considerando $\mu, \alpha$ e $\beta$, com

$$
\begin{aligned}
& R(\alpha \mid \mu, \beta)=R(\mu, \alpha, \beta)-R(\mu, \beta) . \\
& R(\beta \mid \mu, \alpha)=R(\mu, \alpha, \beta)-R(\mu, \alpha) .
\end{aligned}
$$

Finalmente,

$R(\mu, \boldsymbol{\alpha}, \boldsymbol{\beta}, \boldsymbol{\delta})=\boldsymbol{\theta}^{0}{ }^{\prime} X^{\prime} y=S . Q$. Parâmetros do modelo completo.

$$
R(\delta \mid \mu, \alpha, \beta)=R(\mu, \alpha, \beta, \delta)-R(\mu, \alpha, \beta) .
$$

Vale lembrar que a notação e o significado dos termos é o mesmo apresentado em 2.1.2. 


\subsection{Uso de restrições paramétricas para testes de hipóteses}

\subsubsection{Idéias básicas}

Sabe-se, conforme Scheffé (1961), que se a matriz $X$ não é de posto coluna completo, então as soluções dos sistemas de equações normais $\theta^{0}=\left(X^{\prime} X\right)^{G} X^{\prime} y$ variam conforme a escolha da inversa generalizada. Dessa forma, nos modelos de posto incompleto, $\theta^{0}$ não é informativo por si só. Sabe-se também que a aproximação de mínimos quadrados para o vetor $y$ das observações, denominada $\hat{y}=X \theta^{0}$, é invariante para toda e qualquer solução $\theta^{0}$ das equações normais, independentemente da escolha da inversa generalizada. Assim, segundo Iemma (1985) e Searle (1987), se $X \boldsymbol{\theta}^{0}$ é invariante para qualquer $\theta^{0}$, será também invariante para subconjuntos determinados do conjunto de todas as possíveis soluções.

Caso deseje-se obter soluções aproximadas sujeitas a restrições de interesse para o sistema de equações lineares $y=X \boldsymbol{\theta}$ inconsistente, deve-se minimizar o erro $e(\boldsymbol{\theta})=y-X \boldsymbol{\theta}$, para o sistema sujeito a restrições lineares do tipo $B^{\prime} \boldsymbol{\theta}=c$.

Portanto, deve-se encontrar um conjunto de soluções que satisfaçam a uma dada restrição a que os parâmetros estão sujeitos. Tais restrições podem ser tomadas arbitrariamente, ou ainda, ter interesse prático. Segundo Iemma (1987), as restrições paramétricas são parte integrante do modelo, afetando os conceitos de estimabilidade e testabilidade e até mesmo a interpretação das hipóteses formuladas.

Dentre os métodos existentes para a imposição de restrições às funções de várias variáveis, Searle (1971) utiliza o método de multiplicadores de Lagrange, também conhecido como método dos multiplicadores indeterminados. Assim, dada uma função $f\left(x_{l}, \ldots, x_{p}\right)$ e uma restrição $R\left(x_{l}, \ldots, x_{p}\right)$, constrói-se a função de Lagrange $L\left(x_{l}, \ldots, x_{p}, l\right)$, ou função objetivo, em que $l$ é multiplicador de Lagrange:

$$
L\left(x_{l}, \ldots, x_{p}, l\right)=f\left(x_{l}, \ldots, x_{p}\right)-l R\left(x_{l}, \ldots, x_{p}\right) .
$$


Sobre ela aplicam-se os procedimentos usuais para estudo de pontos críticos; no presente contexto, um ponto de mínimo.

Sejam neste contexto, como em Iemma (1985):

$$
\left\{\begin{array}{l}
y=X \boldsymbol{\theta}+e(\boldsymbol{\theta}) \\
c=B^{\prime} \boldsymbol{\theta}
\end{array}\right.
$$

em que $B^{\prime} \boldsymbol{\theta}=c$ é consistente por construção.

Busca-se $\hat{\boldsymbol{\theta}}$ tal que

$$
e^{\prime}(\boldsymbol{\theta}) . e(\boldsymbol{\theta})=(y-X \hat{\boldsymbol{\theta}})^{\prime}(y-X \hat{\boldsymbol{\theta}})=\text { mínimo }\left\{(y-X \boldsymbol{\theta})^{\prime}(y-X \boldsymbol{\theta})\right\},
$$

sujeito a restrição $B^{\prime} \boldsymbol{\theta}=c$. Em outras palavras, há interesse em minimizar a soma de quadrados do resíduo.

Para tanto, segundo Searle (1971), utiliza-se $2 l^{\prime}$ como um vetor de multiplicadores de Lagrange (multiplicadores indeterminados) e minimiza-se

$$
L=(y-X \boldsymbol{\theta})^{\prime}(y-X \boldsymbol{\theta})+2 l^{\prime}\left(B^{\prime} \boldsymbol{\theta}-c\right)
$$

em relação aos elementos de $\theta$ e $l$.

Então,

$$
L=y^{\prime} y-2 y^{\prime} X \boldsymbol{\theta}+\boldsymbol{\theta}^{\prime} X^{\prime} X \boldsymbol{\theta}+2 l^{\prime} B^{\prime} \boldsymbol{\theta}-2 l^{\prime} c .
$$

Diferenciando-se em relação a $\theta$ e $l$, têm-se:

$$
\begin{aligned}
& \frac{\partial L}{\partial \theta} \cong-2 X^{\prime} y+2 X^{\prime} X \boldsymbol{\theta}+2 B l \\
& \frac{\partial L}{\partial l} \cong 2 B^{\prime} \boldsymbol{\theta}-2 c .
\end{aligned}
$$

Fazendo $\frac{\partial L}{\partial \theta}=\phi$ e $\frac{\partial L}{\partial l}=\phi$ vem

$$
\left\{\begin{array}{l}
X^{\prime} X \boldsymbol{\theta}+B l=X^{\prime} y \\
B^{\prime} \boldsymbol{\theta}=c
\end{array}\right.
$$

ou, matricialmente,

$$
\left[\begin{array}{c:c}
X^{\prime} X & B \\
\hdashline B^{\prime} & \phi
\end{array}\right]\left[\begin{array}{l}
\boldsymbol{\theta} \\
-l
\end{array}\right]=\left[\begin{array}{c}
X^{\prime} y \\
\hdashline c
\end{array}\right],
$$

que é o sistema de equações normais com a restrição paramétrica $B^{\prime} \theta=c$. 
De acordo com Iemma (1985), quando a restrição $B^{\prime}$ imposta aos parâmetros não é combinação linear das linhas da matriz $X, B^{\prime}$ é dita linearmente independente, e a solução restrita obtida é solução do sistema irrestrito de equações normais, isto é, é solução de mínimos quadrados para $y=X \boldsymbol{\theta}$ inconsistente. Impondo-se uma restrição que seja combinação linear das linhas da matriz $X$, a mesma é dita linearmente dependente e a solução do sistema restrito não é solução do sistema irrestrito e, nesse caso, não é solução de mínimos quadrados para $y=X \Theta$ inconsistente. Nota-se que essa restrição não completa o posto da matriz resultante e, conseqüentemente, o sistema não terá solução única.

Dessa forma, a invariância garantida para os sistemas irrestritos de equações normais quanto à aproximação e ao erro de mínimos quadrados, permanece válida para os sistemas restritos, se e somente se, a restrição $B^{\prime} \theta$ for tomada de modo que $B^{\prime}$ seja linearmente independente das linhas de $X$ e, portanto, de $X^{\prime} X$ (Iemma, 1985).

\subsubsection{Restrições estimáveis nos parâmetros e soma de quadrados do resíduo}

Discute-se a seguir, como em Searle (1971) e Iemma (1985), implicações decorrentes da imposição de uma restrição paramétrica linearmente dependente na soma de quadrados do resíduo.

Seja o modelo $y=X \boldsymbol{\theta}+e$, com o sistema de equações normais com a restrição paramétrica $B^{\prime} \boldsymbol{\theta}$. Tomando $B^{\prime}$ tal que suas linhas sejam combinações lineares das linhas de $X$, e que também seja de posto linha completo, é possível construir o sistema da seguinte forma:

$$
\left\{\begin{array}{l}
X^{\prime} X \tilde{\boldsymbol{\theta}}+B l=X^{\prime} y \\
B^{\prime} \tilde{\boldsymbol{\theta}}=c
\end{array},\right.
$$

idêntico ao que foi apresentado em 2.5.1, exceto por $\tilde{\boldsymbol{\theta}}$, que indica soluções do sistema com restrições paramétricas linearmente dependentes.

Como as linhas de $B^{\prime}$ são combinações lineares das linhas de $X$, existe uma matriz $W$ tal que (Scheffé, 1961): 


$$
X^{\prime} W=B \text {. }
$$

Substituindo (14) em (13), vem:

$$
\left\{\begin{array}{l}
X^{\prime} X \tilde{\boldsymbol{\theta}}+X^{\prime} W l=X^{\prime} y \\
W^{\prime} X \tilde{\boldsymbol{\theta}}=c
\end{array} .\right.
$$

Em (15), pré-multiplicando $X^{\prime} X \tilde{\boldsymbol{\theta}}+X^{\prime} W l=X^{\prime} y$ por $X^{+\prime} \quad$ (transposta da inversa generalizada de Moore-Penrose de $X$ ), vem:

$$
X^{+\prime} X^{\prime} X \tilde{\boldsymbol{\theta}}+X^{+'} X^{\prime} W l=X^{+'} X^{\prime} y .
$$

Aplicando-se as propriedades da Moore-Penrose, como em Hocking (1985):

$$
X \tilde{\boldsymbol{\theta}}+X X^{+} W l=X X^{+} y
$$

e então,

$$
X \tilde{\boldsymbol{\theta}}=X X^{+} y-X X^{+} W l .
$$

Substituindo (16) em (15):

$$
W^{\prime} X X^{+} y-W^{\prime} X X^{+} W l=c .
$$

Usando (14), tem-se que $W=X^{+} B$ e assim (17) fica:

$$
B^{\prime} X^{+} X X^{+} y-B^{\prime} X^{+} X X^{+} X^{+} B l=c \text {. }
$$

Portanto,

$$
\begin{gathered}
B^{\prime} X^{+} y-B^{\prime} X^{+} X^{+\prime} B l=c, \text { e assim } \\
B^{\prime} X^{+} y-B^{\prime}\left(X^{\prime} X\right)^{+} B l=c .
\end{gathered}
$$

Para generalizar esse resultado, é necessário considerar, segundo Iemma (1987), que se as linhas de $B^{\prime}$ são combinações lineares das linhas de $X$, então $B^{\prime}\left(X^{\prime} X\right)^{G} B$ é invariante, qualquer que seja a inversa generalizada de $X^{\prime} X$.

Dessa forma, (18) fica:

$$
B^{\prime} X^{+} y-B^{\prime}\left(X^{\prime} X\right)^{G} B l=c .
$$

Por outro lado, sabe-se que $\theta^{0}=X^{+} y$ é solução do sistema irrestrito. Além disso, $B^{\prime} \boldsymbol{\theta}^{0}$ é invariante para qualquer solução de $y=X \boldsymbol{\theta}$ consistente e indeterminado. Sendo assim, pode-se generalizar (19) para qualquer $\boldsymbol{\theta}^{0}$ solução do sistema irrestrito: 


$$
\begin{aligned}
& B^{\prime} \boldsymbol{\theta}^{0}-B^{\prime}\left(X^{\prime} X\right)^{G} B l=c, \text { ou } \\
& B^{\prime}\left(X^{\prime} X\right)^{G} B l=B^{\prime} \boldsymbol{\theta}^{0}-c .
\end{aligned}
$$

Sendo $B^{\prime}$ de posto linha completo, por hipótese, então $B^{\prime}\left(X^{\prime} X\right)^{G} B$ é positiva definida e, portanto, não singular. Pode-se assim obter o vetor de multiplicadores de Lagrange, como em Searle (1971):

$$
l=\left[B^{\prime}\left(X^{\prime} X\right)^{G} B\right]^{-1}\left(B^{\prime} \theta^{0}-c\right) .
$$

Avaliando o erro de ajustamento devido à aproximação $\tilde{y}=X \tilde{\boldsymbol{\theta}}$, que não é aproximação de mínimos quadrados para $y$, em função da restrição aplicada ser linearmente dependente, tem-se:

$$
\|\tilde{e}\|^{2}=\|y-\tilde{y}\|^{2}=\|y-X \tilde{\boldsymbol{\theta}}\|^{2}
$$

em que $\|\tilde{e}\|^{2}$ é o quadrado da norma euclidiana de $\tilde{e}$.

Usando (16):

$$
\begin{aligned}
\|\tilde{e}\|^{2} & =\left\|y-X X^{+}(y-W l)\right\|^{2}=\left[y-X X^{+}(y-W l)\right]^{\prime}\left[y-X X^{+}(y-W l)\right]= \\
& =y^{\prime} y-y^{\prime} X X^{+} y+l^{\prime} W^{\prime} X X^{+} W l= \\
& =y^{\prime}\left(I-X X^{+}\right) y+l^{\prime} W^{\prime} X X^{+} W l .
\end{aligned}
$$

É fácil ver que $y^{\prime}\left(I-X X^{+}\right) y$ corresponde à $\|\hat{e}\|^{2}$, ou seja, à soma de quadrados do resíduo do sistema $y=X \boldsymbol{\theta}+e$ sem imposição de restrições. Logo,

$$
\|\tilde{e}\|^{2}=\|\hat{e}\|^{2}+l^{\prime} W^{\prime} X X^{+} W l .
$$

Pode-se reescrever (22) fazendo-se $\delta=l^{\prime} W^{\prime} X X^{+} W l$. Portanto,

$$
\|\tilde{e}\|^{2}=\|\hat{e}\|^{2}+\delta \text {. }
$$

Usando (21), tem-se:

$$
\begin{aligned}
\boldsymbol{\delta} & =l^{\prime} W^{\prime} X X^{+} W l \\
& =\left(B^{\prime} \boldsymbol{\theta}^{0}-c\right)^{\prime}\left[B^{\prime}\left(X^{\prime} X\right)^{G} B\right]^{-1}{ }^{\prime} W^{\prime} X X^{+} W\left[B^{\prime}\left(X^{\prime} X\right)^{G} B\right]^{-1}\left(B^{\prime} \boldsymbol{\theta}^{0}-c\right),
\end{aligned}
$$

e sendo $W=X^{+'} B$, vem 


$$
\begin{aligned}
& =\left(B^{\prime} \boldsymbol{\theta}^{0}-c\right)^{\prime}\left[B^{\prime}\left(X^{\prime} X\right)^{G} B\right]^{-1}{ }^{\prime} B^{\prime} X^{+} X X^{+} X^{+{ }^{\prime}} B\left[B^{\prime}\left(X^{\prime} X\right)^{G} B\right]^{-1}\left(B^{\prime} \boldsymbol{\theta}^{0}-c\right) \\
& =\left(B^{\prime} \boldsymbol{\theta}^{0}-c\right)^{\prime}\left[B^{\prime}\left(X^{\prime} X\right)^{G} B\right]^{-1} B^{\prime} X^{+} X^{+^{\prime}} B\left[B^{\prime}\left(X^{\prime} X\right)^{G} B\right]^{-1}\left(B^{\prime} \boldsymbol{\theta}^{0}-c\right)
\end{aligned}
$$

Assim,

$$
\boldsymbol{\delta}=\left(B^{\prime} \boldsymbol{\theta}^{0}-c\right)^{\prime}\left[B^{\prime}\left(X^{\prime} X\right)^{G} B\right]^{-1}{ }^{\prime}\left(B^{\prime} \boldsymbol{\theta}^{0}-c\right) .
$$

Pode-se então escrever

$$
\begin{gathered}
S Q_{\tilde{\mathrm{Re}} s}=S Q_{\mathrm{Re} s}+\delta, \\
\text { em que: }
\end{gathered}
$$

$S Q_{\tilde{\mathrm{Re}} s}$ : soma de quadrados do resíduo devida ao modelo com a restrição $B^{\prime} \boldsymbol{\theta}=c$ nos parâmetros, para $B^{\prime} \theta$ estimável e $B^{\prime}$ de posto linha completo;

$S Q_{\mathrm{Re} s}$ : soma de quadrados usual do resíduo do modelo irrestrito;

$\boldsymbol{\delta}=\left(B^{\prime} \boldsymbol{\theta}^{0}-c\right)^{\prime}\left[B^{\prime}\left(X^{\prime} X\right)^{G} B\right]^{-1}{ }^{\prime}\left(B^{\prime} \boldsymbol{\theta}^{0}-c\right)$ : acréscimo verificado na $S Q_{\operatorname{Re} s}$, motivado pela imposição da restrição linearmente dependente $B^{\prime} \boldsymbol{\theta}=c$ aos parâmetros do modelo.

Segundo Iemma (1987), testar uma hipótese é equivalente a impor uma restrição estimável aos parâmetros do modelo. Nesse contexto, $B^{\prime}$ pode ser tomada de forma que $H_{0}: B^{\prime} \boldsymbol{\theta}=c$ corresponda a uma hipótese qualquer, de interesse do pesquisador. Assim, tem-se na técnica da restrição paramétrica estimável um critério alternativo para obter somas de quadrados de hipóteses $\left(S Q_{H_{0}}\right)$, tendo em vista que, quando a hipótese é testável, $\delta=S Q_{H_{0}}$. A estatística $F$ para o teste de hipóteses pelo critério alternativo é obtida tomando-se $F\left(H_{0}\right)=\frac{\delta}{r[B] \hat{\sigma}^{2}}$, em que $r[B]$ é o posto de $B$. De acordo com Searle (1971), e com o que foi abordado em 2.3.1,

$$
\left.\frac{\delta}{\sigma^{2}} \sim \chi_{\{r[B]}^{2}, \frac{\left(B^{\prime} \Theta^{0}-c\right)\left[B^{\prime}\left(X^{\prime} X\right){ }^{G} B\right]^{-1}\left(B^{\prime} \Theta^{0}-c\right)}{2 \sigma^{2}}\right\}, \mathrm{e}
$$


$\delta$ e $S Q_{\operatorname{Re} s}$ são independentemente distribuídas. Os significados dos termos são os mesmos já apresentados.

\subsection{A abordagem do SAS para testes de hipóteses}

\subsubsection{Funções estimáveis}

No modelo linear (1) apresentado em 2.1.1, em que $E[y]=X \boldsymbol{\theta}$, um dos interesses da análise é estimar os elementos de $\boldsymbol{\theta}$ (modelo de posto coluna completo) ou alguma combinação linear de seus elementos (caso geral). Como abordado, para estimarse uma função linear específica de $\boldsymbol{\theta}$, diga-se, $L \boldsymbol{\theta}$, deve-se obter uma combinação linear dos componentes do vetor $y$ que tenha como valor esperado $L \boldsymbol{\theta}$.

De acordo com o manual do SAS (SAS Institute, 1989), qualquer combinação linear do vetor $y$ de observações, $k y$, terá como valor esperado $k X \boldsymbol{\theta}$, pois $E(k y)=k X \Theta$. Assim, o valor esperado de qualquer combinação linear dos componentes do vetor $y$ é igual à mesma combinação linear das linhas de $X$ multiplicadas por $\boldsymbol{\theta}$. Pela definição de funções básicas estimáveis de Scheffé (1961), LO é estimável se e somente se uma combinação linear das linhas de $X$ que é igual a $L$ puder ser encontrada.

Dessa forma, as linhas da matriz $X$ formam um conjunto gerador a partir do qual $L$ pode ser construída. Segundo Graybill (1961), Searle (1971) e o SAS Institute (1989), $X$ pode ser reconstruída das linhas de $X^{\prime} X$ pois $X=\left[X\left(X^{\prime} X\right)^{G} X^{\prime} X\right]$ e dessa forma as linhas de $X^{\prime} X$ também formam um conjunto gerador. Tendo-se uma matriz $L$ formada a partir de um conjunto gerador, $L \boldsymbol{\theta}$ será estimável.

Uma vez que $L$, de posto linha completo, tenha sido formada por um conjunto gerador, estima-se $L \boldsymbol{\theta}$ por $L \boldsymbol{\theta}^{0}$. Assim, o teorema de Gauss-Markov (Scheffé, 1961) garante que $L \boldsymbol{\theta}^{0}$ é o BLUE de $L \boldsymbol{\theta}$. 
O módulo GLM do SAS (SAS Institute, 1989) opta por calcular uma inversa generalizada $g 2$ de $X^{\prime} X$, devido ao fato que $\left(X^{\prime} X\right)^{g 2}\left(X^{\prime} X\right)$ contém, em geral, um grande número de zeros, e suas linhas não nulas são linearmente independentes. A matriz $L=\left(X^{\prime} X\right)^{g 2} X^{\prime} X$ é um caso particular da matriz $H=\left(X^{\prime} X\right)^{G} X^{\prime} X$, sendo que a inversa generalizada $\left(X^{\prime} X\right)^{g 2}$ é única para o processo de obtenção do SAS. Logo, $H$ fornece um único $L$, que é um dos conjuntos de funções estimáveis obtidos por $H$.

O procedimento GLM do SAS ("PROC GLM") fornece automaticamente quadros com os coeficientes da forma geral e dos quatro tipos de funções estimáveis, denominadas tipos I, II, III e IV, relacionadas com as hipóteses a serem testadas, assunto que será discutido posteriormente.

De acordo com Mondardo (1994), na forma geral das funções estimáveis, considerando o conjunto modelo-dados, para a obtenção da matriz inversa generalizada $\left(X^{\prime} X\right)^{g 2}$, tomam-se seqüencialmente as colunas linearmente independentes da matriz $X$ como sendo a matriz $X_{1}$ e calcula-se $\left(X_{1}^{\prime} X_{1}\right)^{-1}$. A matriz $\left(X^{\prime} X\right)^{g 2}$ é obtida colocando-se as colunas da matriz $\left(X_{1}^{\prime} X_{1}\right)^{-1}$ em lugar das colunas linearmente independentes e intercalando-se com zeros as demais colunas.

A forma geral das funções estimáveis fornecidas pelo SAS é então obtida por LO e envolve todos os parâmetros do modelo e a cada linha não nula linearmente independente de $\left(X^{\prime} X\right)^{g 2} X^{\prime} X$ é associado um símbolo $L$. O número de símbolos representa o posto máximo da hipótese associada.

Segundo Mondardo \& Iemma (1998), os quadros com os coeficientes das funções estimáveis fornecidas pelo módulo GLM do SAS podem ser utilizados para o reconhecimento das hipóteses que estão sendo testadas. Isso é particularmente importante nas situações em que ocorre desbalanceamento. 


\subsubsection{Tipos de hipóteses construídas pelo SAS}

Segundo Iemma (1995), dentre os vários tipos de hipóteses existentes, o procedimento GLM do SAS incorporou, no caso de modelos com dois fatores e interação, quatro tipos de hipóteses para testar efeitos de linhas (por exemplo, fator A), quatro para efeitos de colunas (por exemplo, fator B) e uma para a interação, as quais serão discutidas brevemente a seguir:

\section{a) Hipóteses do Tipo I}

A igualdade entre os efeitos de um fator é verificada pelas médias ponderadas dos efeitos desse fator, ajustado somente para os efeitos precedentes. Assim, quando se escrevem as hipóteses no modelo-S, ocorrem parâmetros relativos aos efeitos da interação e de outros fatores.

\section{b) Hipóteses do Tipo II}

A igualdade entre os efeitos de um fator é verificada por meio das médias ponderadas dos efeitos desse fator, ajustada para os demais efeitos dos fatores descritos no modelo. O ajuste elimina da forma explícita os efeitos dos parâmetros do fator ajustado.

\section{c) Hipóteses do Tipo III}

São construídas como função de médias não ponderadas dos efeitos de um fator, ajustadas para os efeitos de todos os demais fatores descritos no modelo. Não são consideradas as frequiências das caselas. 


\section{d) Hipóteses do Tipo IV}

Hipóteses dedicadas às situações em que há ocorrência de caselas vazias, sendo considerada como função de médias não ponderadas e dependentes do número de fatores, do número de níveis de cada fator e da quantidade e posição das caselas vazias.

Geralmente, as hipóteses do tipo IV são iguais as do tipo III, porém, segundo Iemma (1995), se existem caselas vazias e mais de dois níveis do fator, então, em geral, as hipóteses dos tipos III e IV são diferentes.

Sua construção, utilizando-se o modelo de médias de caselas, consiste na formação de contrastes entre médias de caselas que estão na mesma coluna (linha), começando pela última linha (coluna). Alterando a posição das linhas em que estão dispostos os dados, será obtida outra hipótese. Assim sendo, pode ser interpretada como um tipo de hipótese que procura auxiliar o pesquisador nos testes de hipóteses quando há caselas vazias, mas isso nem sempre é possível.

\section{e) Hipóteses sobre linhas e colunas}

Como apresentado por Searle (1987), se não há caselas vazias, então para $a$ níveis do fator $A$ e $b$ níveis do fator $B$, têm-se $\frac{1}{4} a b(a-1)(b-1)$ interações possíveis, dentre as quais $(a-1)(b-1)$ são linearmente independentes. Porém, com caselas vazias, existem menos do que $\frac{1}{4} a b(a-1)(b-1)$ interações que podem ser estimadas, e pode ocorrer que menos de $(a-1)(b-1)$ sejam linearmente independentes, modificando o número de graus de liberdade da interação. Segundo Iemma (1991) há, em geral, exceto por desconexões, $(a-1)(b-1)-\mathrm{V}$ interações estimáveis linearmente independentes, em que $\mathrm{v}$ é o número de caselas vazias.

Considerando-se novamente o modelo $y_{i j k}=\mu_{i j}+e_{i j k} \quad$ (3.5) em que $\mu_{i j}=\mu+\alpha_{i}+\beta_{j}$, cada componente da interação é dado por: 


$$
\gamma_{i j, i^{\prime} j^{\prime}}=\mu_{i j}-\mu_{i^{\prime} j}-\mu_{i j^{\prime}}+\mu_{i^{\prime} j^{\prime}}
$$

Quando todas as caselas estão ocupadas a hipótese sobre a interação testa se todas as interações são nulas. Contudo, quando há caselas vazias, há interações que não fazem parte da hipótese. Segundo Iemma (1993), se existem caselas vazias, o teste para interação não deve ser critério para a escolha entre modelos com e sem interação, sendo que essa escolha deve ser baseada na experiência do pesquisador.

De acordo com Speed et al. (1978), utilizando-se o modelo de médias $(y=W \mu+e)$, geralmente de posto completo, as hipóteses discutidas anteriormente podem ser agrupadas conforme a Tabela 1 .

Tabela 1. Forma geral das hipóteses $\left(H_{0}\right)$ para linhas, colunas e interação, com as respectivas somas de quadrados na notação $R($ ) .

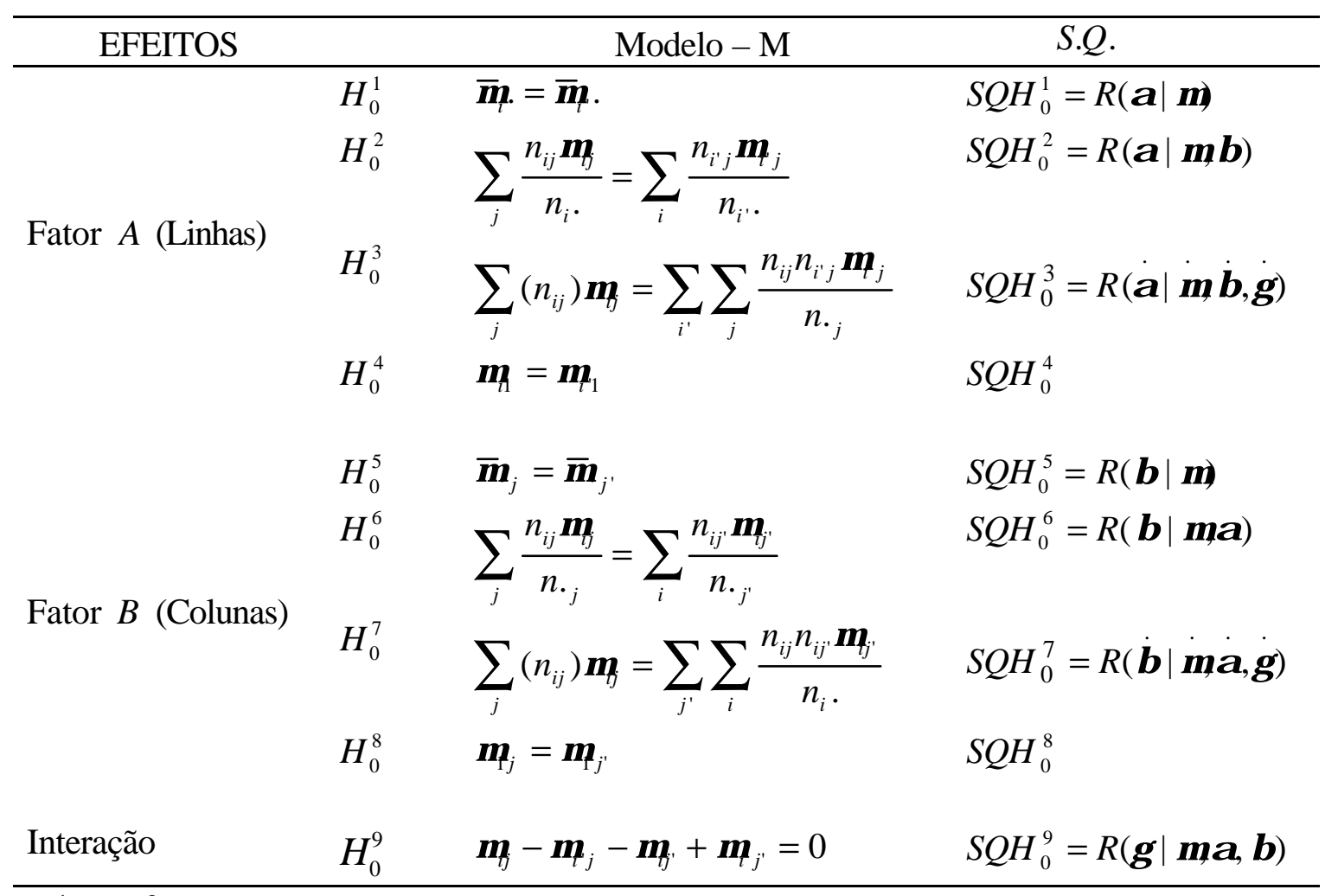

$H_{0}^{1}, \ldots, H_{0}^{9}$ : diferentes hipóteses possíveis; $\bar{\mu}_{i}:$ média ponderada das caselas da linha $i ; n_{i j}$ : frequiência da casela $i j ; \mu_{i j}$ : média da casela $i j ; n_{i}$.: soma das freqüências das caselas da linha $i ; n_{\cdot j}$ : soma das frequiências das caselas da coluna $j ; \bar{\mu}_{. j}$ : média ponderada das caselas da coluna $j ; \mu$ : constante 
inerente a todas as observações; $\alpha$ : efeito do fator $A ; \beta$ : efeito do fator $B$; $\gamma$ : efeito da interação $A \times B ; \dot{\alpha}, \dot{\beta}, \dot{\gamma}$ : o ponto indica que o efeito do fator está ajustado para todos os outros efeitos considerados no modelo.

Os autores ressaltam que nessas hipóteses a descrição do efeito de linha (ou coluna) ou efeito principal de $A$ (ou de $B$ ) não são suficientes para descrever o que se está testando.

\subsubsection{Obtenção das somas de quadrados}

Overall \& Spiegel (1969) generalizaram e formalizaram os principais métodos para análise de dados desbalanceados descritos até então, como em Yates (1934) e Henderson (1953), dentre outros. Esses autores propuseram três diferentes métodos que podem ser apropriados sob diferentes circunstâncias. Em 1976 esses métodos foram incorporados pelo sistema estatístico SAS-76, fazendo parte do módulo GLM (Speed et al., 1978). Nas tabelas 2, 3 e 4 apresentadas a seguir, as hipóteses testadas referem-se à forma geral apresentada na Tabela 1, e apresentam detalhes sobre os quatro tipos de hipóteses testadas pelo SAS.

\section{a) Somas de quadrados do Tipo I}

São obtidas por meio do método da ordenação a priori de Overall \& Spiegel (1969). Podem ser consideradas um caso especial do método do ajustamento de constantes proposto por Yates (1934), no qual se usa $R(\alpha \mid \mu)$ e $R(\beta \mid \mu, \alpha)$ como somas de quadrados dos efeitos principais para linhas e colunas, respectivamente, podendo-se usar alternativamente $R(\beta \mid \mu)$ e $R(\alpha \mid \mu, \beta)$. Segundo Overall \& Spiegel (1969), a ordenação a priori é adequada se existir uma ordenação lógica para os efeitos que estão sendo testados ou, quando se têm dados desbalanceados, com a ordenação de maior interesse para o pesquisador. Dessa forma, estas somas de quadrados são 
dependentes da ordem dos parâmetros no modelo, pois cada efeito é ajustado somente para os efeitos precedentes. São usadas para testar hipóteses sobre médias ponderadas, ajustadas ou não (Tabela 2).

Tabela 2. Somas de quadrados do tipo I, obtidas no SAS para análise de variância de experimentos em um modelo com dois fatores cruzados.

\begin{tabular}{lccl}
\hline Ordenação & Variações Consideradas & Hipóteses Testadas & $S Q I-R()$ \\
\hline \multirow{2}{*}{$A^{*}, B^{* *}, A \mathbf{x} B^{* * *}$} & $A$ & $H_{0}^{1}$ & $R(\boldsymbol{\alpha} \mid \boldsymbol{\mu})$ \\
& $B$ & $H_{0}^{6}$ & $R(\beta \mid \mu, \boldsymbol{\alpha})$ \\
& $A \times B$ & $H_{0}^{9}$ & $R(\gamma \mid \mu, \boldsymbol{\alpha}, \boldsymbol{\beta})$ \\
& $B$ & & \\
$B, A, A \mathbf{x} B$ & $A$ & $H_{0}^{5}$ & $R(\beta \mid \mu)$ \\
& $A \times B$ & $H_{0}^{2}$ & $R(\boldsymbol{\alpha} \mid \mu, \boldsymbol{\beta})$ \\
& & $H_{0}^{9}$ & $R(\gamma \mid \mu, \boldsymbol{\alpha}, \boldsymbol{\beta})$ \\
\hline
\end{tabular}

"Fator $A ;{ }^{* *}$ Fator $B ;{ }^{* * *}$ Interação entre os fatores $A$ e $B ; \mu$ : constante inerente a todas as observações; $\alpha$ : efeito do fator $A ; \beta$ : efeito do fator $B ; \gamma$ : efeito da interação $A \times B$.

No PROC GLM do SAS, quando o conjunto de dados é desbalanceado, a ordem dos fatores no modelo tem grande importância na formulação das hipóteses do tipo I, que é sequiencial: o primeiro fator é ajustado somente em relação à constante, enquanto o segundo é ajustado em relação à constante e ao primeiro fator.

\section{b) Somas de quadrados do Tipo II}

Testam hipóteses sobre médias ponderadas ajustadas. Correspondem na notação $R$ () ao caso em que cada efeito é ajustado para todos os demais efeitos, exceto para interações (Tabela 3). Ressalta-se que, segundo Santos (1994), enquanto para as somas de quadrados do tipo I tem-se:

$$
\begin{aligned}
& R(\mu)+R(\alpha \mid \mu)+R(\beta \mid \mu, \alpha)+R(\gamma \mid \mu, \alpha, \beta)=R(\mu, \alpha, \beta, \gamma) \\
& R(\mu)+R(\beta \mid \mu)+R(\alpha \mid \mu, \beta)+R(\gamma \mid \mu, \alpha, \beta)=R(\mu, \beta, \alpha, \gamma)
\end{aligned}
$$

para as somas de quadrados do tipo II, isto em geral não ocorre, ou seja, 


$$
R(\mu)+R(\alpha \mid \mu)+R(\beta \mid \mu, \alpha)+R(\gamma \mid \mu, \alpha, \beta) \neq R(\mu, \alpha, \beta, \gamma)
$$

devido ao fato das somas de quadrados do tipo II nem sempre serem provenientes de uma partição ortogonal da soma de quadrados dos parâmetros.

Tabela 3. Somas de quadrados do tipo II, para análise de variância de experimentos em um modelo com dois fatores cruzados.

\begin{tabular}{lccl}
\hline Ordenação & Variações Consideradas & Hipóteses Testadas & $S Q I I-R()$ \\
\hline \multirow{2}{*}{$A^{*}, B^{* *}, A \mathbf{x} B^{* * * *}$} & $A$ & $H_{0}^{2}$ & $R(\boldsymbol{\alpha} \mid \boldsymbol{\mu}, \boldsymbol{\beta})$ \\
& $B$ & $H_{0}^{6}$ & $R(\boldsymbol{\beta} \mid \mu, \boldsymbol{\alpha})$ \\
& $A \times B$ & $H_{0}^{9}$ & $R(\gamma \mid \mu, \boldsymbol{\alpha}, \boldsymbol{\beta})$ \\
& $B$ & & \\
$B, A, A \mathbf{x} B$ & $A$ & $H_{0}^{6}$ & $R(\beta \mid \mu, \boldsymbol{\alpha})$ \\
& $A \times B$ & $H_{0}^{2}$ & $R(\boldsymbol{\alpha} \mid \mu, \boldsymbol{\beta})$ \\
& & $H_{0}^{9}$ & $R(\gamma \mid \mu, \boldsymbol{\alpha}, \boldsymbol{\beta})$ \\
\hline
\end{tabular}

"Fator $A ;{ }^{* *}$ Fator $B ;{ }^{* * *}$ Interação entre os fatores $A$ e $B ; \mu$ : constante inerente a todas as observações; $\alpha$ : efeito do fator $A ; \beta$ : efeito do fator $B ; \gamma$ : efeito da interação $A \times B$.

Nesse caso, a ordem dos parâmetros no modelo não influi, pois cada efeito é ajustado para todos os demais efeitos, exceto para a interação.

\section{c) Somas de quadrados do Tipo III}

Podem ser obtidas através dos métodos dos quadrados de médias ponderadas de Yates (1934) ou de mínimos quadrados completos de Overall \& Spiegel (1969), entre outros. As hipóteses associadas a essas somas de quadrados dizem respeito às médias não ponderadas de linhas e/ou colunas (Tabela 4). Nesse caso tem-se o ajuste de um parâmetro em relação aos demais e, dessa forma, a ordem dos parâmetros do modelo não tem importância. 
Tabela 4. Somas de quadrados do tipo III, para análise de variância de experimentos em um modelo com dois fatores cruzados.

\begin{tabular}{lccc}
\hline Ordenação & Variações Consideradas & Hipóteses Testadas & $S Q I I I-R()$ \\
\hline$A^{*}, B^{* *}, A \times B^{* * * *}$ & $A$ & $H_{0}^{3}$ & $R(\dot{\alpha} \mid \dot{\mu}, \dot{\beta}, \dot{\gamma})$ \\
& $B$ & $H_{0}^{7}$ & $R(\dot{\beta} \mid \dot{\mu}, \dot{\alpha}, \dot{\gamma})$ \\
& $A \times B$ & $H_{0}^{9}$ & $R(\gamma \mid \mu, \alpha, \beta)$ \\
$B, A, A \times B$ & $A$ & $H_{0}^{7}$ & $R(\dot{\beta} \mid \dot{\mu}, \dot{\alpha}, \dot{\gamma})$ \\
& $A \times B$ & $H_{0}^{3}$ & $R(\dot{\alpha} \mid \dot{\mu}, \dot{\beta}, \dot{\gamma})$ \\
& $H_{0}^{9}$ & $R(\gamma \mid \mu, \alpha, \beta)$ \\
\hline
\end{tabular}

"Fator $A ;{ }^{* *}$ Fator $B ;{ }^{* * *}$ Interação entre os fatores $A$ e $B ; \mu$ : constante inerente a todas as observações;

$\dot{\alpha}$ : efeito do fator $A$ ajustado para todos os demais efeitos; $\dot{\beta}$ : efeito do fator $B$ ajustado para todos os demais efeitos; $\gamma$ : efeito da interação $A \times B$ ajustada para todos os demais efeitos.

\section{d) Somas de quadrados do Tipo IV}

As somas de quadrados do tipo IV testam hipóteses que são construídas pelo PROC GLM do SAS, sendo função de médias não ponderadas. Elas dependem da posição e do número de caselas vazias. Assim, se existem caselas vazias, as somas de quadrados do tipo IV podem não ser únicas.

Para verificar que hipóteses são geradas e testadas faz-se necessário o exame das funções estimáveis do tipo IV. Se o conjunto de dados não apresenta casela vazia, as somas de quadrados do tipo IV são iguais às do tipo III. Existindo pelo menos uma casela vazia, então as somas de quadrados dos tipos III e IV são, em geral, diferentes e referem-se a diferentes hipóteses (Iemma, 1995). 


\subsubsection{As funções CONTRAST e ESTIMATE}

No PROC GLM do SAS, existem as funções CONTRAST e ESTIMATE com as quais, respectivamente, faz-se testes de hipóteses sobre combinações lineares das médias dos níveis e estimam-se contrastes. Dessa forma, esse é um mecanismo que permite ao usuário testar facilmente hipóteses de seu interesse. Assim, de acordo com o SAS Institute (1989), serão discutidos a seguir alguns aspectos referentes a essas funções.

\section{a) Função CONTRAST}

Proporciona um mecanismo para obter facilmente teste de hipóteses usuais, de interesse do pesquisador, tais como $H_{0}: B^{\prime} \boldsymbol{\theta}=\boldsymbol{\phi}$. O teste é realizado por meio do vetor ou matriz $B^{\prime}$, que é a matriz com os coeficientes da função paramétrica $B^{\prime} \theta$, sendo que $r\left[B^{\prime}\right]$ é definido pelos graus de liberdade da hipótese. Para uma hipótese não testável, o PROC GLM informa que a função não é estimável. Quando a hipótese é testável, ou seja, quando sua função paramétrica é estimável, sua soma de quadrados $\left(S Q_{H_{0}: B^{\prime} \Theta=\phi}\right)$ é calculada por:

$$
S Q_{H_{0}: B^{\prime} \Theta=\phi}=\left(B^{\prime} \Theta^{0}\right)^{\prime}\left[B\left(X^{\prime} X\right)^{g 2} B^{\prime}\right]^{-1}\left(B^{\prime} \Theta^{0}\right)
$$

em que

$\boldsymbol{\theta}^{0}=\left(X^{\prime} X\right)^{g 2} X^{\prime} y$;

$\left(X^{\prime} X\right)^{g 2}$ : inversa generalizada $g 2 \mathrm{de}\left(X^{\prime} X\right)$.

Essa soma de quadrados é calculada, sendo que a matriz $B$ deve ser de posto coluna completo. Em caso contrário, a soma de quadrados é calculada usando-se uma matriz $B$, eliminando-se as linhas linearmente dependentes. 


\section{b) Função ESTIMATE}

Utilizada para estimar funções lineares dos parâmetros, multiplicando-se o vetor $B$ pelo vetor de parâmetros estimado $\boldsymbol{\theta}^{0}$, ou seja, calculando $B^{\prime} \boldsymbol{\theta}^{0}$. A estimabilidade da função linear é verificada por meio de $B^{\prime}$, ou seja, verificando se $B^{\prime}$ é linearmente dependente ou independente. A estimativa $B^{\prime} \boldsymbol{\theta}^{0}$, em que $\boldsymbol{\theta}^{0}=\left(X^{\prime} X\right)^{g 2} X^{\prime} y$, é apresentada com seu erro padrão associado, $\sqrt{B^{\prime}\left(X^{\prime} X\right)^{g 2} B \hat{\sigma}^{2}}$ e teste $t$ de Student para $B^{\prime} \boldsymbol{\theta}=\boldsymbol{\phi}$.

2.6.5 Considerações sobre o PROC GLM do SAS para análise de dados desbalanceados

Dentre os sistemas estatísticos que contêm bons procedimentos consagrados à análise de experimentos com modelos de classificação dupla e dados desbalanceados, destaca-se o sistema estatístico SAS ("Statistical Analysis System”) (SAS Institute, 1989) e em particular o procedimento GLM ("General Linear Model Procedure"), dedicado à análise de modelos de efeitos fixos.

Conforme apresentado, o PROC GLM dispõe de quatro tipos de hipóteses para cada fator e para cada interação descrita no modelo. Assim, conforme Searle (1987), o usuário tem a vantagem de poder optar pela hipótese mais adequada aos objetivos de sua pesquisa.

Muitos pesquisadores têm demonstrado preocupação com a complexidade das hipóteses testadas na análise de variância com amostras desbalanceadas e com caselas vazias. Segundo Iemma (1993), a ocorrência de caselas vazias pode conduzir a alguns problemas em relação à estimação de funções paramétricas e, portanto, a testabilidade dessas funções. Esses problemas refletem-se sobre a interpretação das verdadeiras hipóteses testadas pelas somas de quadrados. São eles:

a) podem ocorrer desconexões, ou seja, a impossibilidade de se estimar certas combinações lineares dos parâmetros do modelo correspondente (Searle, 1987); 
b) o teste da interação, em geral, testa somente se algumas interações são nulas;

c) as hipóteses sobre médias não ponderadas de linhas e colunas, em geral, têm uma interpretação bastante diferente daquelas com caselas ocupadas;

d) a interpretação das hipóteses depende da posição e do número de caselas vazias;

e) os números de graus de liberdade da interação devem ser escolhidos com cautela.

Utilizando modelos lineares para dados desbalanceados, Santos (1994) discute sobre a estruturação de hipóteses e compara as saídas geradas por diversos sistemas estatísticos, visando uma orientação na análise e formulação das hipóteses. Nessa mesma linha, Mondardo (1994) alerta os pesquisadores para a complexidade da análise de dados desbalanceados com caselas vazias e procura orientá-los na escolha do método de análise mais adequado.

Por meio de situações práticas, Camarinha Filho (1995) discute sobre os erros que podem ser cometidos na interpretação das hipóteses quando os dados são desbalanceados e apresentam caselas vazias. A interpretação das hipóteses mais comumente testadas pelo procedimento GLM do SAS é abordada em detalhes por Iemma (1995). Já os mecanismos utilizados pelo PROC GLM do SAS para construção dos quatro tipos de funções estimáveis e, portanto, das hipóteses testáveis são discutidos por Mondardo (1994) e Mondardo \& Iemma (1998).

Segundo Camarinha Filho (1995), quando se escrevem em termos do modelo superparametrizado as hipóteses testadas pelos quatro tipos de somas de quadrados, fornecidas pelo PROC GLM do SAS, todos os tipos de hipóteses envolvem os parâmetros referentes à interação, não se podendo testar os efeitos principais isoladamente, caso a interação seja significativa. Portanto, somente a hipótese sobre a interação é livre de parâmetros sem interesse. 


\subsection{Variáveis auxiliares}

Scott \& Milliken (1993) propuseram uma técnica, usando variáveis auxiliares, para decomposição dos graus de liberdade de tratamentos na realização de análises de variância usando o PROC GLM do SAS. Nesse trabalho é abordada a análise de delineamentos em blocos aumentados, baseada no método descrito por Federer (1956). Utilizaram-se quatro cultivares de soja (denominados tratamentos comuns) repetidos três vezes num delineamento em blocos completos casualizados. Adicionalmente, 17 cultivares não repetidas (tratamentos regulares) foram incluídas no experimento, sendo colocados seis cultivares nos dois primeiros blocos e cinco no terceiro bloco. A parcela restante no terceiro bloco foi completada duplicando-se um tratamento "comum" em cada bloco.

A técnica de análise usando variável auxiliar consistiu em definir duas novas variáveis na construção do modelo: a variável $C$, que tem o nome dos tratamentos comuns quando se refere a esses tratamentos e zero em caso contrário, e a variável $X$, que tem o nome dos tratamentos regulares quando se refere a eles, ou zero em caso contrário. Portanto, o modelo assume a forma:

$$
y_{i j}=\mu+b_{j}+C_{i}+X_{i}\left(C_{i}\right)+e_{i j}
$$

em que

$y_{i j}$ : valor observado para o $i$-ésimo tratamento no $j$-ésimo bloco;

$b_{j}$ : efeito do bloco $j$;

$C_{i}$ : efeito do tratamento comum;

$X_{i}$ : efeito do tratamento adicional;

$e_{i j}$ : erro experimental.

Assim, a soma de quadrados para $C$ fornece um teste de hipótese de que as médias dos tratamentos comuns não diferem dos tratamentos adicionais. A soma de quadrados de $X(C)$ está relacionada a um teste da hipótese de que todas as médias dos 
tratamentos adicionais são iguais. Essa última é de particular interesse no melhoramento genético.

Perecin et. al (2000) apresentaram exemplos do uso de variáveis auxiliares para expressar desdobramentos de graus de liberdade e contrastes utilizando o sistema estatístico SAS. Nesse trabalho os autores mostram que as variáveis auxiliares podem ser utilizadas para modelos de experimentos fatoriais e de parcelas subdivididas, quando a interação for significativa. Além disso, mostram também que com essa técnica é possível ajustar modelos de regressão quando uma variável tem níveis quantitativos. 


\section{MATERIAL E MÉTODOS}

\subsection{Material}

\subsubsection{Conjunto A}

Em um experimento de competição entre dois grupos de variedades de cana-deaçúcar, utilizou-se o delineamento inteiramente casualizado com 6 tratamentos e 5 repetições. Os resultados, em t/ha, são apresentados na Tabela 5. Os tratamentos foram divididos em dois grupos, sendo o grupo 1 composto pelas variedades CB 5034 e CB 6245 e o grupo 2 composto pelas variedades IAC $62-58$, IAC $65-29$, IAC $68-14$ e IAC 65 - 38. O objetivo desse experimento é testar se as variedades CB (grupo 1) diferem das variedades IAC (grupo 2), bem como se há diferenças entre as variedades dentro de cada grupo.

Tabela 5. Produtividade de 5 variedades de cana-de-açúcar (t/ha), em 5 repetições, no delineamento inteiramente casualizado.

\begin{tabular}{llcccc}
\hline VARIEDADES & \multicolumn{5}{c}{ REPETIÇÕES } \\
\hline (1) CB 5034 & 112,3 & 121,0 & 114,3 & 115,8 & 117,2 \\
(2) CB 6245 & 125,3 & 119,7 & 120,8 & 120,5 & 122,3 \\
(3) IAC 62 - 58 & 118,4 & 120,5 & 119,7 & 118,3 & 117,8 \\
(4) IAC 65 - 29 & 127,9 & 128,3 & 129,5 & 126,5 & 127,3 \\
(5) IAC 68 - 14 & 130,1 & 122,4 & 126,7 & 127,3 & 128,9 \\
(6) IAC 65 - 38 & 115,2 & 123,2 & 117,8 & 120,8 & 116,4 \\
\hline
\end{tabular}

Fonte: Adaptado de Campos (1984). 


\subsubsection{Conjunto B}

Os dados da Tabela 6 referem-se à produtividade, em t/ha, obtidos de um experimento fictício de competição entre híbridos de milho, em que foi utilizado o delineamento blocos casualizados. Os tratamentos utilizados foram: híbridos simples HS, híbridos duplos - HD e híbridos triplos - HT. O interesse dessa pesquisa está em verificar se há diferença de produtividade entre os três grupos de híbridos e entre os híbridos dentro de cada grupo.

Tabela 6. Produtividade de híbridos de milho (t/ha), no delineamento em blocos casualizados.

\begin{tabular}{ccccc}
\hline & & \multicolumn{3}{c}{ BLOCOS } \\
HÍBRIDOS & 1 & 2 & 3 & 4 \\
\hline (1) HS1 & 3,4 & 3,0 & 3,1 & 3,2 \\
(2) HS2 & 2,7 & 2,9 & 2,8 & 3,0 \\
(3) HS3 & 2,6 & 2,5 & 2,1 & 2,4 \\
(4) HD1 & 2,9 & 3,5 & 3,2 & 3,3 \\
(5) HD2 & 2,6 & 2,8 & 3,0 & 3,1 \\
(6) HD3 & 2,3 & 2,6 & 2,2 & 3,0 \\
(7) HT1 & 3,1 & 3,3 & 3,4 & 2,2 \\
(8) HT2 & 2,0 & 1,9 & 2,2 & 3,1 \\
(9) HT3 & 3,2 & 3,4 & 3,3 & \\
\hline
\end{tabular}

Fonte: Dados fictícios.

\subsubsection{Conjunto C}

Os dados desse conjunto resultam de um experimento fatorial $2 \times 3$, inteiramente ao acaso, com 4 repetições, sendo cada unidade experimental formada por 5 unidades de observação (explantes), para testar os efeitos de 3 reguladores de crescimento (hormônios) na concentração $6 \mu M$, sobre a propagação "in vitro" de 2 porta-enxertos (cultivares) de macieira. Cada explante produziu várias brotações que foram medidas da inserção até o ápice. Os hormônios utilizados foram 6-benzilaminopurina (BAP), tiadizurom (TDZ) e forclorfenuro (CPPU) e as cultivares testadas foram M.111 e M.7. Ao final do período de cultivo avaliou-se o comprimento médio das várias brotações 
produzidas, medidas em $\mathrm{cm}$, obtendo-se um conjunto de dados desbalanceados com uma casela vazia, uma vez que para a combinação TDZ com M7 não foi obtida nenhuma medida (Tabela 7). Nesse experimento, o interesse é verificar se há diferenças entre os efeitos de hormônios e entre os efeitos de cultivares, bem como se há interação entre hormônios e cultivares.

Tabela 7. Comprimento médio de brotos de explantes $(\mathrm{cm})$, medidos em duas cultivares de porta-enxerto de macieira, tratados com três hormônios de crescimento.

\begin{tabular}{|c|c|c|c|c|}
\hline & & \multicolumn{3}{|c|}{ Fator A (Hormônios) } \\
\hline & & BAP & CPPU & TDZ \\
\hline \multirow{8}{*}{$\begin{array}{c}\text { Fator B } \\
\text { (Cultivares) }\end{array}$} & \multirow{4}{*}{ M111 } & - & 0,322 & - \\
\hline & & 1,204 & 0,382 & - \\
\hline & & 1,256 & 0,220 & 0,272 \\
\hline & & 1,300 & - & 0,240 \\
\hline & \multirow{4}{*}{ M7 } & 1,828 & 0,282 & - \\
\hline & & 2,186 & - & - \\
\hline & & 1,336 & 0,256 & - \\
\hline & & 2,232 & 0,404 & - \\
\hline
\end{tabular}

Fonte: Adaptado de Nesi (2000).

\subsection{Métodos}

\subsubsection{Modelo Linear de Gauss-Markov Normal}

Adotou-se para os três conjuntos de dados, segundo Iemma (1987), o modelo linear de efeitos fixos dado por

$$
y=X \boldsymbol{\theta}+e,
$$

em que:

${ }_{n} y_{1}$ : vetor de observações;

${ }_{n} X_{p}$ : matriz de "uns" e "zeros" que identifica o delineamento experimental;

${ }_{p} \boldsymbol{\Theta}_{1}$ : vetor de parâmetros desconhecidos;

${ }_{n} e_{1}$ : vetor de variáveis aleatórias não observáveis, tal que $e \sim N\left(\phi, I \sigma^{2}\right)$. 
Assumiu-se em todos os casos que os resíduos são independentes e normalmente distribuídos, com variância comum $\sigma^{2}$. Em cada conjunto de dados, de acordo com a situação, o modelo assumiu diferentes caracterizações e desdobramentos, conforme será apresentado.

\subsubsection{Análise do conjunto A}

\subsubsection{Hipóteses testadas automaticamente pelo PROC GLM do SAS}

Utilizando-se o PROC GLM do SAS, fez-se inicialmente uma análise de variância convencional, como em Pimentel Gomes (1990). Para tanto, adotou-se o modelo linear superparametrizado, caracterizado por:

$$
y_{i j}=\mu+\tau_{i}+e_{i j}
$$

em que

$y_{i j}$ : valor observado para o $i$-ésimo tratamento na $j$-ésima repetição;

$\mu$ : constante inerente a todas as observações;

$\tau_{i}$ : efeito do $i$-ésimo tratamento (variedade);

$e_{i j}$ : erro aleatório relativo à observação $y_{i j}$.

A análise de variância convencional testa a hipótese de que os tratamentos não diferem entre si. Como o conjunto de dados é balanceado, as hipóteses construídas a partir de qualquer um dos quatro tipos das funções estimáveis fornecidas pelo SAS são equivalentes, resultando em somas de quadrados iguais. Para os dados da Tabela 5, são 6 tratamentos, resultando em uma hipótese com 5 graus de liberdade. A hipótese testada tem a forma:

$$
H_{0}: \mu_{1}=\mu_{2} \cdot=\mu_{3}=\mu_{4}=\mu_{5}=\mu_{6}
$$

em que:

$\mu_{1} . \therefore$ média de produção da variedade 1 ;

$\mu_{2}$. : média de produção da variedade 2 ; 
$\mu_{3} .:$ média de produção da variedade 3 ;

$\mu_{4}$. : média de produção da variedade 4;

$\mu_{5}$. : média de produção da variedade 5;

$\mu_{6}$. : média de produção da variedade 6.

Para o conjunto de dados e modelo adotado, o esquema de análise de variância é (Tabela 8):

Tabela 8. Análise de variância para o modelo $y_{i j}=\mu+\tau_{i}+e_{i j}$.

\begin{tabular}{ccccc}
\hline V.C.M.* & G.L. & S.Q. & Q.M. & F \\
\hline Variedades & $(t-1)$ & $S Q_{H_{0}}=R(\tau \mid \mu)$ & $\frac{R(\tau \mid \mu)}{t-1}$ & $\frac{Q M_{\text {Variedade }}}{Q M_{\text {Re síduo }}}$ \\
Resíduo & $(n-t)$ & $R(\mu, \tau)-R(\tau \mid \mu)$ & $\frac{R(\mu, \tau)-R(\tau \mid \mu)}{n-t}$ & - \\
Total & $(n-1)$ & $R(\mu, \tau)$ & - & -
\end{tabular}

* Variações consideradas no modelo; $\mu$ : efeito da constante; $\tau$ : efeito dos tratamentos; $t:$ número de tratamentos (variedades); $n:$ número de observações.

O programa utilizado no SAS para realização da análise de variância encontra-se no APÊNDICE.

\subsubsection{Testes de hipóteses de interesse usando contrastes}

Como um dos objetivos desse experimento é obter informações com relação à comparação entre variedades, uma alternativa para obter essas informações é decompor os graus de liberdade de tratamentos em contrastes ortogonais, e testá-los utilizando a função CONTRAST do PROC GLM. No experimento da Tabela 5, para as 6 variedades, definiu-se um conjunto com 5 contrastes ortogonais $\left(\begin{array}{lll}Y_{1} & \text { a } & Y_{5}\end{array}\right)$ que representam as comparações que interessam ao experimentador: 
i) Grupo 1 vs. Grupo 2 (1 g.l.):

$$
Y_{1}: 2 \mu_{1} \cdot+2 \mu_{2} \cdot-\mu_{3} \cdot-\mu_{4} \cdot-\mu_{5} \cdot-\mu_{6} .=0
$$

ii) Variedade 1 vs. Variedade 2 (1 g.l.):

$$
Y_{2}: \mu_{1} \cdot-\mu_{2} \cdot=0
$$

iii) Variedades 3 e 4 vs. Variedades 5 e 6 (1 g.l.):

$$
Y_{3}: \mu_{3} \cdot+\mu_{4} \cdot-\mu_{5} \cdot-\mu_{6}=0
$$

iv) Variedade 3 vs. Variedade 4 (1g.1.):

$$
Y_{4}: \mu_{3} \cdot-\mu_{4}=0
$$

v) Variedade 5 vs. Variedade 6 ( 1 g.l.):

$$
Y_{5}: \mu_{5} \cdot-\mu_{6}=0
$$

Evidentemente, dependendo do interesse do experimentador, outro poderia ser o grupo de contrastes ortogonais.

A função CONTRAST realiza o teste dessas hipóteses calculando $B^{\prime}$, que é o vetor com os coeficientes da função paramétrica. A soma de quadrados para cada hipótese foi calculada por

$$
S Q_{H_{0}}=\left(B^{\prime} \boldsymbol{\theta}^{0}\right)^{\prime}\left[B\left(X^{\prime} X\right)^{g 2} B^{\prime}\right]^{-1}\left(B^{\prime} \boldsymbol{\theta}^{0}\right) .
$$

O teste dessa hipótese foi feito usando 


$$
\frac{\frac{S Q_{H_{0}}}{r[B]}}{\frac{S Q_{\operatorname{Re} s}}{(n-r[X])}} \sim F_{\left\{r[B], n-r[X], \frac{\left(B^{\prime} \boldsymbol{\theta}^{0}\right)^{\prime}\left[B^{\prime}\left(X^{\prime} X\right)^{G} B\right]^{-1}\left(B^{\prime} \boldsymbol{\theta}^{0}\right)}{2 \sigma^{2}}\right\}}
$$

em que

$S Q_{H_{0}}$ : soma de quadrados relativa à hipótese $H_{0}$;

$B$ : matriz com os coeficientes da função paramétrica correspondente à $H_{0}$;

$r[B]:$ posto da matriz $B$;

$S Q_{\operatorname{Re} s}:$ soma de quadrados do resíduo;

$n$ : número de observações;

$X$ : matriz do delineamento;

$r[X]$ : posto da matriz $X$;

$$
\begin{gathered}
\theta^{0}=\left(X^{\prime} X\right)^{G} X^{\prime} y \text { : solução do sistema de equações normais; } \\
\left(X^{\prime} X\right)^{g 2}: \text { inversa generalizada } g 2 \text { de } X^{\prime} X
\end{gathered}
$$

A hipótese de diferença das variedades do grupo 1 foi testada com o contraste $Y_{2}$. Por sua vez, a diferença entre as variedades do grupo 2 foi testada construindo $B^{\prime}$ formado pelos coeficientes de $Y_{3}, Y_{4}$ e $Y_{5}$, tendo portanto 3 graus de liberdade.

O programa SAS utilizado pode ser visto no APÊNDICE.

\subsubsection{Variáveis auxiliares}

No experimento em questão, como há interesse em testar se as variedades CB diferem das IAC, foram definidos dois grupos de tratamentos. Assim, para realizar a análise de variância considerando essa divisão em grupos, adaptou-se a técnica proposta por Scott \& Milliken (1993), para análise de experimentos em blocos aumentados. Essa técnica foi utilizada de forma semelhante por Perecin et al. (2000) para realizar desdobramentos de graus de liberdade e calcular contrastes usando o SAS. 
No presente trabalho, essa técnica consistiu em se codificar os tratamentos de acordo com os grupos de interesse. A codificação foi feita utilizando-se novas variáveis, aqui denominadas variáveis auxiliares. Para esse experimento foram utilizadas duas variáveis auxiliares, tendo em vista que são dois grupos de variedades (CB e IAC). As variáveis auxiliares foram assim definidas (programa SAS no APÊNDICE):

i) variável $C$ : assume o nome das variedades do grupo 1 (CB), quando o tratamento corresponde as variedades $\mathrm{CB}$ e zero em caso contrário.

ii) variável $D$ : assume o nome das variedades do grupo 2 (IAC), quando o tratamento corresponde às variedades IAC e zero em caso contrário.

Assim, a caracterização do modelo linear apresentada em (26) foi reescrita em função das variáveis auxiliares. Dessa forma tem-se:

$$
y_{i j}=\mu+c_{i}+d_{i}+e_{i j}
$$

em que

$y_{i j}$ : valor observado para o $i$-ésimo tratamento na $j$-ésima repetição;

$\mu$ : constante inerente a todas as observações;

$c_{i}$ : efeito das variedades do grupo 1 ;

$d_{i}$ : efeito das variedades do grupo 2 ;

$e_{i j}$ : erro aleatório relativo à observação $y_{i j}$.

Nota-se que a caracterização do modelo linear sem a definição de $C$ e de $D$ é $y_{i j}=\mu+\tau_{i}+e_{i j}$, apresentado em (26). Com as variáveis auxiliares, $\tau_{i}$ foi expresso como soma de dois efeitos: $\tau_{i}=c_{i}+d_{i}$.

Matricialmente, as caracterizações (26) e (27) podem ser escritas na forma $y=X \boldsymbol{\theta}+e$, como em (25), em que os vetores $y$ e $e$ são iguais em ambas. Entretanto, as matrizes de delineamento $(X)$ e os vetores de parâmetros $\boldsymbol{\theta}$ diferem, já que na caracterização (27), a parte referente aos tratamentos da caracterização (26), está subdividida nos efeitos $c_{i}$ e $d_{i}$.

Os testes de hipóteses para as variedades auxiliares $C$ e $D$ foram feitos como apresentados a seguir: 
i) Hipótese de que as médias das variedades do grupo 1 não diferem entre si (teste para a variável auxiliar $C$ ):

$$
H_{0}: \mu_{1}=\mu_{2}
$$

ii) Hipótese de que as médias das variedades do grupo 2 não diferem entre si (teste para a variável auxiliar $D$ ):

$$
H_{0}: \mu_{3}=\mu_{4}=\mu_{5}=\mu_{6} .
$$

Nesses dois casos, a hipótese foi testada diretamente na análise de variância usando a forma geral da soma de quadrados da hipótese já apresentada. O esquema da análise de variância é apresentado na Tabela 9.

Tabela 9. Análise de variância do modelo $y_{i j}=\mu+c_{i}+d_{i}+e_{i j}$.

\begin{tabular}{ccccc}
\hline V.C.M. & G.L. & S.Q. & Q.M. & F \\
\hline$C$ & $\left(c^{\prime}-1\right)$ & $S Q_{H_{0}}=R(c \mid \mu, d)$ & $\frac{R(c \mid \mu, d)}{c^{\prime}-1}$ & $\frac{Q M_{C}}{Q M_{\text {Re síduo }}}$ \\
$D$ & $\left(d^{\prime}-1\right)$ & $S Q_{H_{0}}=R(d \mid \mu, c)$ & $\frac{R(d \mid \mu, c)}{d^{\prime}-1}$ & $\frac{Q M_{D}}{Q M_{\text {Re síduo }}}$ \\
Resíduo & $(n-t)$ & $R(\mu, \tau)-R(\tau \mid \mu)$ & $\frac{R(\mu, \tau)-R(\tau \mid \mu)}{n-t}$ & - \\
Total & $(n-t)$ & $R(\mu, \tau)$ & - & - \\
\hline
\end{tabular}

$\mu$ : constante; $c_{i}$ : efeito das variedades do grupo $1 ; d_{i}$ : efeito das variedades do grupo $2 ; c^{\prime}:$ número de níveis que assume a variável auxiliar $C ; d^{\prime}$ : número de níveis que assume a variável auxiliar $D ; n$ : número de observações; $t$ : número de tratamentos.

Nesse caso, o uso da variável auxiliar não proporciona um mecanismo para testar o contraste entre o grupo 1 e o grupo 2. Isso foi contornado construindo-se esse contraste e testando-o pela função CONTRAST do PROC GLM, de forma idêntica ao que foi apresentado em 3.2.2.2. 
O programa em SAS utilizado para realizar essa análise de variância (Tabela 9) encontra-se no APÊNDICE.

\subsubsection{Análise do conjunto B}

\subsubsection{Hipóteses testadas automaticamente pelo SAS}

Para o conjunto de dados B (Tabela 6), utilizando-se o PROC GLM de forma convencional, fez-se uma análise de variância como em Pimentel Gomes (1990), calculando-se somas de quadrados para blocos, tratamentos e resíduo.

Assim, adotou-se como caracterização para o modelo linear superparametrizado:

$$
\begin{gathered}
y_{i j}=\mu+\beta_{j}+\tau_{i}+e_{i j} \\
\text { em que }
\end{gathered}
$$

$y_{i j}$ : valor observado para o $i$-ésimo tratamento no $j$-ésimo bloco;

$\mu$ : constante inerente a todas as observações;

$\beta_{j}$ : efeito do $j$-ésimo bloco;

$\tau_{i}:$ efeito do $i$-ésimo tratamento (híbrido);

$e_{i j}$ : erro aleatório relativo à observação $y_{i j}$.

Nesse caso, a análise de variância feita automaticamente pelo SAS testa a hipótese de que os kíbridos não diferem entre si. Pelo fato de o conjunto de dados ser balanceado, as hipóteses construídas a partir dos quatro tipos de funções estimáveis são equivalentes, resultando em somas de quadrados iguais. Para os dados da Tabela 6, como são 9 tratamentos (9 híbridos), construiu-se uma hipótese com 8 graus de liberdade:

$$
H_{0}: \mu_{1} .=\mu_{2} .=\mu_{3} .=\mu_{4} .=\mu_{5} .=\mu_{6} .=\mu_{7} .=\mu_{8} .=\mu_{9} ., \text { em que }
$$

$\mu_{1} .:$ média de produção de HS1;

$\mu_{2} .:$ média de produção de HS2; 
$\mu_{3} .:$ média de produção de HS3;

$\mu_{4} .:$ média de produção de HD1;

$\mu_{5} .:$ média de produção de HD2;

$\mu_{6} .:$ média de produção de HD3;

$\mu_{7} .:$ média de produção de HT1;

$\mu_{8} .:$ média de produção de HT2;

$\mu_{9} .:$ média de produção de HT3;

Um esquema da análise de variância pode ser visto na Tabela 10, sendo que o programa SAS está apresentado no APÊNDICE.

Tabela 10. Análise de variância do modelo $y_{i j}=\mu+\beta_{j}+\tau_{i}+e_{i j}$.

\begin{tabular}{ccccc}
\hline V.C.M. & G.L. & S.Q. & Q.M. & F \\
\hline Blocos & $(b-1)$ & $S Q_{\text {Blocos }}=R(\beta \mid \mu, \tau)$ & $\frac{R(\beta \mid \mu, \tau)}{b-1}$ & - \\
Híbridos & $(t-1)$ & $S Q_{H_{0}}=R(\tau \mid \mu, \beta)$ & $\frac{R(\tau \mid \mu, \beta)}{t-1}$ & $\frac{Q M_{\text {Hibridos }}}{Q M_{\text {Residuo }}}$ \\
& & $\Delta$ & $\frac{\Delta}{n-t}$ & - \\
Resíduo & $(n-t)$ & $R(\mu, \beta, \tau)$ & - & - \\
Total & $(n-1)$ & \multicolumn{2}{c}{. } &
\end{tabular}

\subsubsection{Testes de hipóteses de interesse usando contrastes}

Como há interesse em verificar quais híbridos diferem entre si, foram decompostos os 8 graus de liberdade para híbridos em contrastes ortogonais, os quais foram testados com o uso da função CONTRAST do PROC GLM. Para tanto, têm-se os 
seguintes contrastes ortogonais, que representam as comparações que interessam ao pesquisador $\left(Y_{1}, \ldots, Y_{8}\right)$ :

i) Híbridos simples vs. Híbridos duplos, Híbridos triplos (1 g.l.):

$$
Y_{1}: 2 \mu_{1} .+2 \mu_{2} .+2 \mu_{3} \cdot-\mu_{4} \cdot-\mu_{5} \cdot-\mu_{6} \cdot-\mu_{7} .-\mu_{8} .-\mu_{9}=0
$$

ii) Híbridos simples 1 vs. Híbridos simples 2 e 3 (1 g.l.):

$$
Y_{2}: 2 \mu_{1} \cdot-\mu_{2} \cdot-\mu_{3} .=0
$$

iii) Híbridos simples 2 vs. Híbridos simples 3 (1 g.l.):

$$
Y_{3}: \mu_{2} \cdot-\mu_{3} .=0
$$

iv) Híbridos duplos vs. Híbridos triplos (1 g.l.):

$$
Y_{4}: \mu_{4} \cdot+\mu_{5} \cdot+\mu_{6 \cdot}-\mu_{7} \cdot-\mu_{8} \cdot-\mu_{9}=0
$$

v) Híbrido duplo 4 vs. Híbridos duplos 5 e 6 (1 g.l.):

$$
Y_{5}: 2 \mu_{4} \cdot-\mu_{5} \cdot-\mu_{6} .=0
$$

vi) Híbrido duplo 5 vs. Híbrido duplo 6 (1 g.l.):

$$
Y_{6}: \mu_{5} \cdot-\mu_{6} \cdot=0
$$


vii) Híbrido triplo 7 vs. Híbridos triplos 8 e 9:

$$
Y_{7}: 2 \mu_{7} \cdot-\mu_{8} \cdot-\mu_{9} \cdot=0
$$

viii) Híbrido triplo 8 vs. Híbrido triplo 9 (1 g.l.)

$$
Y_{8}: \mu_{8} \cdot-\mu_{9}=0
$$

Obviamente, dependendo do interesse, podem ser construídos outros conjuntos de contrastes ortogonais.

Utilizando a função CONTRAST, o teste dessas hipóteses foi realizado por meio de $B^{\prime}$, que é o vetor com os coeficientes da função paramétrica (Programa SAS no APÊNDICE). Assim, a soma de quadrados para os contrastes é calculada por

$$
S Q_{Y_{i}}=\left(B^{\prime} \boldsymbol{\theta}^{0}\right)^{\prime}\left[B\left(X^{\prime} X\right)^{g 2} B^{\prime}\right]^{-1}\left(B^{\prime} \boldsymbol{\theta}^{0}\right),
$$

com significados já apresentados. O teste de hipóteses foi feito considerando-se

$$
\frac{\frac{S Q_{H_{0}}}{r[B]}}{\frac{S Q_{\operatorname{Re} s}}{(n-r[X])}} \sim F_{\left\{r[B], n-r[X], \frac{\left(B^{\prime} \theta^{0}\right)^{\prime}\left[B^{\prime}(X ' X)^{G} B\right]^{-1}\left(B^{\prime} \boldsymbol{\theta}^{0}\right)}{2 \sigma^{2}}\right\}} .
$$

A diferença entre os híbridos simples foi testada construindo um $B^{\prime}$ formado pelos coeficientes de $Y_{2}$ e $Y_{3}$ (2 graus de liberdade). O teste para a diferença entre os híbridos duplos foi feito usando $B^{\prime}$ formado pelos coeficientes de $Y_{5}$ e $Y_{6}$ (2 graus de liberdade) e para a diferença de híbridos triplos, $B^{\prime}$ teve os coeficientes de $Y_{7}$ e $Y_{8}$ (2 graus de liberdade).

\subsubsection{Variáveis auxiliares}

Para esse experimento, como há interesse em testar se os híbridos simples (HS), duplos (HD) e triplos (HT) diferem, os tratamentos foram divididos em três grupos. 
Considerando-se os grupos para realizar a análise de variância, foi utilizada a técnica da variável auxiliar, assim como para o conjunto de dados A. Agora, fez-se a codificação usando três variáveis auxiliares $(C, D$ e $F)$, sendo que (programa SAS no APÊNDICE):

i) variável $C$ : assume o nome dos híbridos simples quando o tratamento corresponde a esses híbridos, e zero caso contrário.

ii) variável $D$ : assume o nome dos híbridos duplos, quando o tratamento corresponde a esses híbridos e zero caso contrário.

iii) variável $F$ : assume o nome dos híbridos triplos quando o tratamento corresponde a esses híbridos e zero caso contrário.

Assim, a caracterização apresentada em (28) foi reescrita em função das variáveis auxiliares:

$$
y_{i j}=\mu+\beta_{j}+c_{i}+d_{i}+f_{i}+e_{i j}
$$

em que

$y_{i j}$ : valor observado para o $i$-ésimo híbrido no $j$-ésimo bloco;

$\mu$ : constante inerente a todas as observações;

$\beta_{j}$ : efeito do $j$-ésimo bloco;

$c_{i}$ : efeito dos híbridos simples (grupo 1);

$d_{i}$ : efeito dos híbridos duplos (grupo 2);

$f_{i}$ : efeito dos híbridos triplos (grupo 3);

$e_{i j}$ : erro aleatório relativo à observação $y_{i j}$.

As caracterizações (28) e (29) também podem ser escritas na forma matricial $y=X \boldsymbol{\theta}+e$, como em (25), em que os vetores $y$ e $e$ são iguais em todos os modelos. Há diferenças entre as matrizes do delineamento $(X)$ e o vetor de parâmetros $\theta$, de acordo com as variáveis auxiliares utilizadas na codificação. $\mathrm{O}$ efeito de tratamentos pode ser expresso como a soma de três efeitos: 


$$
\tau_{i}=c_{i}+d_{i}+f_{i}
$$

Os testes de hipóteses para as variáveis auxiliares foram utilizados da seguinte forma:

i) Hipótese de que as médias dos híbridos simples não diferem entre si (variável auxiliar $C$ ):

$$
H_{0}: \mu_{1}=\mu_{2}=\mu_{3}
$$

ii) Hipótese de que as médias dos híbridos duplos não diferem entre si (variável auxiliar $D$ ):

$$
H_{0}: \mu_{4}=\mu_{5}=\mu_{6}
$$

iii) Hipótese de que as médias dos híbridos triplos não diferem entre si (variável auxiliar $F$ ):

$$
H_{0}: \mu_{7} \cdot=\mu_{8} \cdot=\mu_{9}
$$

O teste dessas hipóteses pode ser agrupado conforme a Tabela 11 (programa SAS no APÊNDICE). 
Tabela 11. Análise de variância do modelo $y_{i j}=\mu+\boldsymbol{\beta}_{j}+c_{i}+d_{i}+f_{i}+e_{i j}$.

\begin{tabular}{ccccc}
\hline V.C.M. & G.L. & S.Q. & Q.M. & F \\
\hline Blocos & $(b-1)$ & $S Q_{\text {Blocos }}=R(\beta \mid \mu, \tau)$ & $\frac{R(\beta \mid \mu, \tau)}{b-1}$ & - \\
$C$ & $\left(c^{\prime}-1\right)$ & $S Q H_{0}=R(c \mid \mu, \beta, d, f)$ & $\frac{R(c \mid \mu, \beta, d, f)}{\left(c^{\prime}-1\right)}$ & $\frac{Q M_{C}}{Q M_{\text {Re síduo }}}$ \\
$D$ & $\left(d^{\prime}-1\right)$ & $S Q H_{0}=R(d \mid \mu, \beta, c, f)$ & $\frac{R(d \mid \mu, \beta, c, f)}{\left(d^{\prime}-1\right)}$ & $\frac{Q M_{D}}{Q M_{\text {Re síduo }}}$ \\
$F$ & $\left(f^{\prime}-1\right)$ & $S Q H_{0}=R(f \mid \mu, \beta, c, d)$ & $\frac{R(f \mid \mu, \beta, c, d)}{\left(f^{\prime}-1\right)}$ & $\frac{Q M_{F}}{Q M_{\text {Re síduo }}}$ \\
Resíduo & $(n-t)$ & $\Delta$ & $\frac{\Delta}{n-t}$ & - \\
Total & $(n-1)$ & $R(\mu, \beta, \tau)$ & - & - \\
\hline V.C.M. vaç & & &
\end{tabular}

V.C.M.: variações consideradas no modelo; $\mu$ : constante; $\beta$ : efeito de blocos; $\tau$ : efeito dos tratamentos; $C$ : efeito dos híbridos simples; $D$ : efeito dos híbridos duplos; $F$ : efeito dos híbridos triplos; $\Delta=R(\mu, \beta, \tau)-R(\beta \mid \mu, \tau)-R(\tau \mid \mu, \beta) ; b$ : número de blocos; $c^{\prime}$ : número de níveis que assume a variável auxiliar $C ; d^{\prime}$ : número de níveis que assume a variável auxiliar $D ; f^{\prime}$ : número de níveis que assume a variável auxiliar $F ; t$ : número de tratamentos (híbridos); $n:$ número de observações.

De forma análoga ao que ocorreu para o conjunto A, o uso das variáveis auxiliares não permitiu que fossem testadas as diferenças entre os três grupos, o que foi feito com o uso da função CONTRAST, de forma idêntica ao apresentado em 3.2.3.2. 


\subsubsection{Análise do conjunto $\mathrm{C}$}

\subsubsection{Caracterizações do modelo linear}

a) Modelo de média de caselas

O modelo-M (média de caselas) adotado para o experimento fatorial com dois fatores cruzados, foi caracterizado por:

$$
\begin{gathered}
y_{i j k}=\mu_{i j}+e_{i j k}, \\
\text { em que: }
\end{gathered}
$$

$y_{i j k}$ : valor da $k$-ésima repetição para o $i$-ésimo nível do hormônio (fator $A$ ) e $j$-ésimo nível de cultivar (fator $B$ );

$\mu_{i j}$ : média da população da qual foi extraída a amostra que compõe a casela $(i, j)$;

$e_{i j k}:$ erro aleatório atribuído à observação $y_{i j k}$, tal que $e_{i j k} \sim N\left(\phi, I \sigma^{2}\right)$;

$i=1,2,3$ níveis de hormônios;

$j=1,2$ níveis de cultivares;

$k=1,2, \ldots, n_{i j}$ observações na casela $(i, j)$.

\section{b) Modelo superparametrizado}

Para o experimento em questão, o modelo linear com dois fatores cruzados foi caracterizado como em Graybill (1961), Searle (1971) e Iemma (1995):

$$
y_{i j k}=\mu+\alpha_{i}+\beta_{j}+\gamma_{i j}+e_{i j k},
$$

para a ordenação $A-B$ e

$$
y_{i j k}=\mu+\beta_{j}+\alpha_{i}+\gamma_{i j}+e_{i j k},
$$

para a ordenação $B$ - $A$, em que: 
$y_{i j k}$ : valor da $k$-ésima repetição no $i$-ésimo nível do fator $A$ e $j$-ésimo nível do fator $B$

$\mu$ : constante inerente a todas as observações;

$\alpha_{i}$ : efeito do $i$-ésimo nível do fator $A$;

$\beta_{j}$ : efeito do $j$-ésimo nível do fator $B$;

$\gamma_{i j}$ : efeito da interação entre o i-ésimo nível do fator $A$ e o j-ésimo nível do fator $B$;

$e_{i j k}$ : erro aleatório atribuído à observação $y_{i j k}$, tal que $e_{i j k} \sim N\left(\phi, I \sigma^{2}\right)$.

A ordem dos fatores no modelo foi considerada na formulação das hipóteses e obtenção das somas de quadrados, pois o conjunto de dados é desbalanceado e apresenta casela vazia.

\subsubsection{Hipóteses estatísticas testadas pelo PROC GLM do SAS}

Para mostrar como foram construídas as hipóteses, com base no modelo-M, os dados da Tabela 7 foram esquematizados em função do número de observações em cada casela (Tabela 12):

Tabela 12. Esquema dos dados do conjunto C (Tabela 7) em relação ao modelo-M.

\begin{tabular}{cccc}
\hline & \multicolumn{3}{c}{$\operatorname{HORMÔNIOS}(A)$} \\
CULTIVARES $(B)$ & $i=1$ & $i=2$ & $i=3$ \\
\hline$j=1$ & $\mu_{11}^{(3)} *$ & $\mu_{21}^{(3)}$ & $\mu_{31}^{(2)}$ \\
$j=2$ & $\mu_{12}^{(4)}$ & $\mu_{22}^{(3)}$ & - \\
\hline
\end{tabular}

* O número entre parênteses descreve a freqüência da casela; $\mu_{i j}$ : média da casela $(i, j) ; i$ : níveis do fator $A ; j$ : níveis do fator $B$.

Em seguida, foram construídas as hipóteses estatísticas testadas pelo PROC GLM do SAS, decorrentes das suas funções estimáveis e denominadas tipos I, II, III e IV para os efeitos principais, $A$ (Colunas), $B$ (Linhas) e interação ( $A \times B)$. 


\subsection{Hipóteses sobre hormônios (Fator A, colunas)}

A hipótese do tipo I refere-se a igualdade entre os efeitos de hormônios, verificada por meio de suas médias ponderadas.

No presente caso, teve a seguinte forma:

$$
H_{0}^{1-A}:\left\{\begin{array}{l}
\frac{3}{7} \mu_{11}+\frac{4}{7} \mu_{12}-\mu_{31}=0 \\
\frac{1}{2} \mu_{21}+\frac{1}{2} \mu_{22}-\mu_{31}=0
\end{array} .\right.
$$

A soma de quadrados para essa hipótese, foi obtida por:

$$
S Q_{H_{0}^{1-A}}=R(\alpha \mid \mu) .
$$

A hipótese do tipo II testa a igualdade entre os efeitos de hormônios, sendo verificada por meio de médias ponderadas de colunas ajustadas para linhas. No caso:

$$
H_{0}^{2-A}:\left\{\begin{array}{l}
\frac{11}{15} \mu_{11}+\frac{4}{15} \mu_{12}+\frac{4}{15} \mu_{21}-\frac{4}{15} \mu_{22}-\mu_{31}=0 \\
\frac{11}{15} \mu_{11}-\frac{4}{15} \mu_{12}+\frac{4}{15} \mu_{21}+\frac{4}{15} \mu_{22}-\mu_{31}=0
\end{array}\right.
$$

A soma de quadrados respectiva foi dada por:

$$
S Q_{H_{0}^{2-A}}=R(\boldsymbol{\alpha} \mid \boldsymbol{\mu}, \boldsymbol{\beta}) .
$$

Para a hipótese do tipo III, testa-se a igualdade entre os efeitos de hormônios por meio de médias não ponderadas. No caso, 


$$
H_{0}^{3-A}:\left\{\begin{array}{l}
\frac{3}{4} \mu_{11}+\frac{1}{4} \mu_{12}+\frac{1}{4} \mu_{21}-\frac{1}{4} \mu_{22}-\mu_{31}=0 \\
\frac{1}{4} \mu_{11}-\frac{1}{4} \mu_{12}+\frac{3}{4} \mu_{21}+\frac{1}{4} \mu_{22}-\mu_{31}=0
\end{array}\right. \text {. }
$$

Utilizando a notação $R($ ), tem-se como soma de quadrados para essa hipótese:

$$
S Q_{H_{0}^{3-A}}=R(\dot{\alpha} \mid \dot{\mu}, \dot{\beta}, \dot{\gamma})
$$

A hipótese do tipo IV, sobre igualdade entre os efeitos de hormônios, foi verificada por meio de médias não ponderadas. No esquema apresentado na Tabela 12, trocaram-se as linhas pelas colunas e construíram-se contrastes entre médias de caselas que estão na mesma coluna, iniciando-se pela última linha, obtendo:

$$
H_{0}^{4-A}:\left\{\begin{array}{l}
\mu_{11}-\mu_{31}=0 \\
\mu_{21}-\mu_{31}=0
\end{array} .\right.
$$

A soma de quadrados para a hipótese do tipo IV não tem relação com a notação $R()$, como as demais.

\subsection{Hipóteses sobre cultivares (Fator B, linhas)}

De modo análogo ao discutido para hormônios, obtiveram-se hipóteses sobre cultivares, agora com um grau de liberdade e, portanto, hipóteses com uma linha.

A hipótese do tipo I, sobre a igualdade entre os efeitos de cultivares, foi testada por meio de suas médias ponderadas:

$$
H_{0}^{1-B}: \frac{3}{8} \mu_{11}-\frac{4}{7} \mu_{12}+\frac{3}{8} \mu_{21}-\frac{3}{7} \mu_{22}+\frac{1}{4} \mu_{31}=0 .
$$


Pela notação $R($ ), sua soma de quadrados é dada por:

$$
S Q_{H_{0}^{1-B}}=R(\beta \mid \mu) .
$$

Para a hipótese do tipo II, que testa a igualdade entre os efeitos de cultivares por meio de médias ponderadas de linhas ajustadas para colunas, obteve-se:

$$
H_{0}^{2-B}:-\frac{4}{7} \mu_{11}+\frac{4}{7} \mu_{12}-\frac{3}{6} \mu_{21}+\frac{3}{6} \mu_{22}=0 .
$$

Utilizando a notação $R($ ), calculou-se a soma de quadrados para essa hipótese por:

$$
S Q_{H_{0}^{2-B}}=R(\beta \mid \mu, \alpha) .
$$

A hipótese do tipo III, sobre a igualdade entre os efeitos de cultivares, foi verificada por meio de médias não ponderadas:

$$
H_{0}^{3-B}: \frac{1}{2} \mu_{11}-\frac{1}{2} \mu_{12}+\frac{1}{2} \mu_{21}-\frac{1}{2} \mu_{22}=0 .
$$

Na notação $R($ ), a soma de quadrados para essa hipótese foi obtida por:

$$
S Q_{H_{0}^{3-B}}=R(\dot{\beta} \mid \dot{\mu}, \dot{\alpha}, \dot{\gamma})
$$

Para a hipótese do tipo IV, que verifica a igualdade entre os efeitos de cultivares por meio de médias não ponderadas, obteve-se: 


$$
H_{0}^{4-B}: \mu_{11}-\mu_{12}+\mu_{21}-\mu_{22}=0 \text {. }
$$

Assim como para hormônios, a hipótese do tipo IV para cultivares não pode ser escrita em termos da notação $R($ ) como as demais.

\subsection{Hipóteses sobre a interação}

Para o conjunto de dados $\mathrm{C}$, devido à presença de casela vazia, testou-se a seguinte hipótese sobre a interação:

$$
H_{0}^{A x B}: \mu_{11}-\mu_{12}-\mu_{21}+\mu_{22}=0
$$

A soma de quadrados para a interação, foi dada por

$$
S Q_{H_{0}^{A x B}}=R(\gamma \mid \mu, \alpha, \beta)
$$

O esquema da análise de variância pode ser visto na Tabela 13, sendo o programa SAS apresentado no APÊNDICE. 
Tabela 13. Análise de variância do modelo completo para o conjunto C.

\begin{tabular}{|c|c|c|c|c|}
\hline V.C.M. & G.L. & S.Q. & Q.M. & $\mathrm{F}$ \\
\hline Hormônios & $(a-1)$ & $S Q_{H_{0}^{A}}$ & $\frac{S Q_{H_{0}^{A}}}{(a-1)}$ & $\frac{Q M_{\text {Hormônios }}}{Q M_{\text {Re síduo }}}$ \\
\hline Cultivares & $(b-1)$ & $S Q_{H_{0}^{B}}$ & $\frac{S Q_{H_{0}^{B}}}{(b-1)}$ & $\frac{Q M_{\text {Cultivares }}}{Q M_{\text {Re síduo }}}$ \\
\hline Interação & $(a-1)(b-1)-v$ & $S Q_{H_{0}^{A x B}}$ & $\frac{S Q_{H_{0}^{A x B}}}{(a-1)(b-1)-v}$ & $\frac{Q M_{\text {Interação }}}{Q M_{\operatorname{Re} s i d u o}}$ \\
\hline Resíduo & $\Delta_{1}$ & $\Delta_{2}$ & $\frac{\Delta_{2}}{\Delta_{1}}$ & - \\
\hline Total & $(n-1)$ & $\left(y^{\prime} y-C\right)$ & - & - \\
\hline
\end{tabular}

$a$ : níveis do fator $A ; b:$ níveis do fator $B ; \boldsymbol{v}:$ número de caselas vazias; $y$ : vetor de observações;

$$
\Delta_{1}:(n-1)-[(a-1)+(b-1)+(a-1)(b-1)-v] ; \quad \Delta_{2}:\left(y^{\prime} y-C\right)-S Q H_{0}^{A}-S Q H_{0}^{B}-S Q H_{0}^{A x B} ; \quad S Q_{H_{0}}:
$$

representam as somas de quadrados associadas a cada hipótese apresentada; $C:$ correção.

\subsubsection{Hipóteses testadas pe la função CONTRAST}

Algumas hipóteses de interesse do pesquisador foram elaboradas e testadas utilizando-se a função CONTRAST do PROC GLM do SAS. O objetivo dessa análise foi mostrar que é possível, usando a função CONTRAST, criar hipóteses de interesse e verificar sua testabilidade. No caso, optou-se por testar hipóteses equivalentes àquelas do tipo III.

Para o fator A, uma hipótese de interesse foi

$$
H_{0}^{3-A}:\left\{\begin{array}{l}
\frac{3}{4} \mu_{11}+\frac{1}{4} \mu_{12}+\frac{1}{4} \mu_{21}-\frac{1}{4} \mu_{22}-\mu_{31}=0 \\
\frac{1}{4} \mu_{11}-\frac{1}{4} \mu_{12}+\frac{3}{4} \mu_{21}+\frac{1}{4} \mu_{22}-\mu_{31}=0
\end{array}\right. \text {. }
$$


É possível supor que deve haver interesse em verificar o efeito de hormônios sem considerar o efeito de cultivares, ou seja, desconsiderando a interação. Para tanto, elaborou-se a hipótese:

$$
H_{0}^{*-A}:\left\{\begin{array}{rr}
\alpha_{1} & -\alpha_{3}=0 \\
& \alpha_{2}-\alpha_{3}=0
\end{array}\right. \text {. }
$$

Para o fator B:

$$
H_{0}^{3-B}: \frac{1}{2} \mu_{11}-\frac{1}{2} \mu_{12}+\frac{1}{2} \mu_{21}-\frac{1}{2} \mu_{22}=0
$$

Vale ressaltar que não necessariamente as hipóteses de interesse são testáveis, conforme será verificado.

Da mesma forma que para o fator hormônios, pode ser de interesse da pesquisa verificar os efeitos de cultivares sem considerar o efeito de hormônios. Nesse sentido tem-se a seguinte hipótese:

$$
H_{0}^{*-B}: \beta_{1}-\beta_{2}=0
$$

que não é testável, mas foi construída para mostrar como isso é abordado pelo SAS.

A função CONTRAST calcula somas de quadrados para hipóteses de interesse por meio de

$$
S Q_{H_{0}}=\left(B^{\prime} \theta^{0}\right)^{\prime}\left[B\left(X^{\prime} X\right)^{g 2} B^{\prime}\right]^{-1}\left(B^{\prime} \theta^{0}\right),
$$

que é testada por

$$
\frac{\frac{S Q_{H_{0}}}{r[B]}}{\frac{S Q_{\operatorname{Re} s}}{(n-r[X])}} \sim F_{\left\{r[B], n-r[X], \frac{\left(B^{\prime} \mathbf{\Theta}^{0}\right)^{\prime}\left[B^{\prime}\left(X^{\prime} X\right)^{G} B\right]^{-1}\left(B^{\prime} \mathbf{\theta}^{0}\right)}{2 \boldsymbol{\sigma}^{2}}\right\}}, \text { em que: }
$$


$S Q_{H_{0}}$ : soma de quadrados relativa àhipótese $H_{0}$;

$B$ : matriz com os coeficientes da função paramétrica correspondente à $H_{0}$;

$r[B]:$ posto da matriz $B$;

$S Q_{\mathrm{Re} s}:$ soma de quadrados do resíduo;

$n$ : número de observações;

$X$ : matriz do delineamento;

$r[X]$ : posto da matriz $X$;

$$
\begin{gathered}
\theta^{0}=\left(X^{\prime} X\right)^{G} X^{\prime} y \text { : solução do sistema de equações normais; } \\
\left(X^{\prime} X\right)^{g 2} \text { : inversa generalizada } g 2 \text { de } X^{\prime} X .
\end{gathered}
$$

\subsubsection{Hipóteses testadas pela técnica da restrição paramétrica estimável}

As hipóteses apresentadas para a função CONTRAST no item anterior, também foram testadas, quando possível, pelo critério da imposição de restrição paramétrica estimável ao sistema de equações normais. Segundo Iemma (1987), uma hipótese linear geral associada ao conjunto de parâmetros, $H_{0}: B^{\prime} \boldsymbol{\theta}=c$, é testável quando os elementos de $B^{\prime} \boldsymbol{\theta}$ são estimáveis.

De acordo com Nesi \& Iemma (2001), tomando-se uma hipótese testável e empregando-a como uma restrição paramétrica, haverá aumento na soma de quadrados do resíduo. Esse aumento, $\delta$, é uma soma de quadrados devida a restrição, ou seja, associada à hipótese que está sendo testada. A uma restrição linearmente dependente equivale uma função paramétrica estimável e, portanto, uma hipótese testável. Quando a restrição é linearmente independente das linhas de $X$, a função paramétrica não é estimável, e assim, a hipótese correspondente não é testável. Nesse caso, o aumento $\delta$ é zero.

Essas idéias foram usadas para testar hipóteses de interesse, quando possível. Os passos seguidos foram: 
i) Construiu-se o modelo linear superparametrizado na forma matricial, $y=X \boldsymbol{\theta}+e$ e a hipótese $H_{0}: B^{\prime} \boldsymbol{\theta}=\boldsymbol{\phi}$ que se deseja testar.

ii) Construiu-se o sistema de equações normais com a restrição paramétrica:

$$
\left[\begin{array}{c:c}
X^{\prime} X & B \\
\hdashline B^{\prime} & \phi
\end{array}\right]\left[\begin{array}{l}
\theta \\
-l
\end{array}\right]=\left[\begin{array}{c}
X^{\prime} y \\
\hdashline \phi
\end{array}\right],
$$

em que $B^{\prime}$ é a matriz hipótese (restrição) e $\boldsymbol{\theta}$ é um vetor de parâmetros desconhecidos.

iii) Fez-se:

$$
\left[\begin{array}{c}
\tilde{\boldsymbol{\theta}} \\
\hdashline l
\end{array}\right]=\left[\begin{array}{c:c}
X^{\prime} X & B \\
\hdashline B^{\prime} & \phi
\end{array}\right]^{G}\left[\begin{array}{c}
X^{\prime} y \\
\hdashline \phi
\end{array}\right],
$$

em que $G$ é uma inversa generalizada qualquer. Obteve-se $\tilde{\boldsymbol{\theta}}$, que é solução do sistema de equações normais com a restrição paramétrica $B^{\prime} \theta=\phi$.

iv) Calculou-se $\tilde{y}=X \tilde{\Theta}$ (aproximação para o vetor $y$ de observações no sistema de equações normais com a restrição paramétrica), e então, calculou-se o vetor $\tilde{e}$ (erro de ajustamento devido à solução aproximada $\tilde{\boldsymbol{\theta}}$ ) e com ele a soma de quadrados do resíduo para o sistema de equações normais com restrição paramétrica $\left(S Q_{\widetilde{R} e s}\right)$ :

$$
y-\tilde{y}=\tilde{e} \quad \text { e } \quad \tilde{e}^{\prime} \tilde{e}=\|\tilde{e}\|^{2}=S Q_{\tilde{\operatorname{Re}} s} .
$$

v) Com o objetivo de obter a soma de quadrados usual do resíduo, tomou-se $\boldsymbol{\theta}^{0}=\left(X^{\prime} X\right)^{G} X^{\prime} y$, qualquer solução das equações normais $X^{\prime} X \boldsymbol{\theta}=X^{\prime} y$ e, portanto, solução de mínimos quadrados para o sistema $y=X \boldsymbol{\theta}$ inconsistente. Por meio de $\boldsymbol{\theta}^{0}$ obteve-se o vetor $\hat{y}$, aproximação de mínimos quadrados para o vetor $y$ das observações:

$$
\hat{y}=X \boldsymbol{\theta}^{0} \text {. }
$$

vi) Calculou-se o erro de ajustamento $(\hat{e})$ devido à aproximação de mínimos quadrados, para o sistema $y=X \boldsymbol{\theta}$ inconsistente, e com ele a soma de quadrados usual do resíduo $\left(S Q_{\operatorname{Res}}\right)$ :

$$
y-\hat{y}=\hat{e} \quad \text { e } \quad \hat{e}^{\prime} \hat{e}=\|\hat{e}\|^{2}=S Q_{\operatorname{Re} s} .
$$


vii) Verificou-se o acréscimo na soma de quadrados usual do resíduo calculandose a quantidade $\delta$ (Iemma, 1987; Nesi $\&$ Iemma, 2001):

$$
\delta=S Q_{\tilde{\operatorname{Re}} s}-S Q_{\operatorname{Re} s} .
$$

Considerou-se que, caso $\delta=0$, tem-se que a restrição é linearmente independente e, portanto, a função paramétrica não é estimável e, em consequiência, a hipótese não é testável. Se $\delta>0$, a restrição é linearmente dependente e, portanto, a função paramétrica é estimável e, em conseqüência, a hipótese é testável sendo que $\delta=S Q_{H_{0}}$ (soma de quadrados associada à hipótese $\left.H_{0}\right)$.

viii) Nos casos em que $\delta>0$, calculou-se a estatística $\mathrm{F}$ para $H_{0}$ :

$$
F\left(H_{0}\right)=\frac{\delta}{r[B] \hat{\sigma}^{2}} .
$$

Algumas hipóteses de interesse do pesquisador foram elaboradas e testadas utilizando-se a técnica da restrição paramétrica estimável. Assim, mostrou-se que é possível criar hipóteses de interesse e verificar sua testabilidade. No caso, optou-se por testar hipóteses equivalentes àquelas do tipo III.

Para o fator A, uma hipótese de interesse foi

$$
H_{0}^{3-A}:\left\{\begin{array}{l}
\frac{3}{4} \mu_{11}+\frac{1}{4} \mu_{12}+\frac{1}{4} \mu_{21}-\frac{1}{4} \mu_{22}-\mu_{31}=0 \\
\frac{1}{4} \mu_{11}-\frac{1}{4} \mu_{12}+\frac{3}{4} \mu_{21}+\frac{1}{4} \mu_{22}-\mu_{31}=0
\end{array}\right.
$$

Supondo-se interesse em verificar o efeito de hormônios sem considerar o efeito de cultivares, elaborou-se a hipótese: 


$$
H_{0}^{*-A}:\left\{\begin{array}{rr}
\alpha_{1} & -\alpha_{3}=0 \\
& \alpha_{2}-\alpha_{3}=0
\end{array}\right. \text {. }
$$

Para o fator B:

$$
H_{0}^{3-B}: \frac{1}{2} \mu_{11}-\frac{1}{2} \mu_{12}+\frac{1}{2} \mu_{21}-\frac{1}{2} \mu_{22}=0
$$

Da mesma forma que para o fator hormônios, pode ser de interesse da pesquisa verificar os efeitos de cultivares sem considerar o efeito de hormônios. Nesse sentido tem-se a hipótese:

$$
H_{0}^{*-B}: \beta_{1}-\beta_{2}=0
$$

que não é testável, mas foi construída para mostrar como isso é abordado na técnica da restrição paramétrica estimável. 


\section{RESULTADOS E DISCUSSÃO}

\subsection{Conjunto A}

\subsubsection{Hipóteses testadas automaticamente pelo PROC GLM do SAS}

Para o experimento apresentado na Tabela 5, com caracterização do modelo linear dada por $y_{i j}=\mu+\tau_{i}+e_{i j}(26)$, obteve-se matricialmente $(y=X \boldsymbol{\theta}+e)$ :

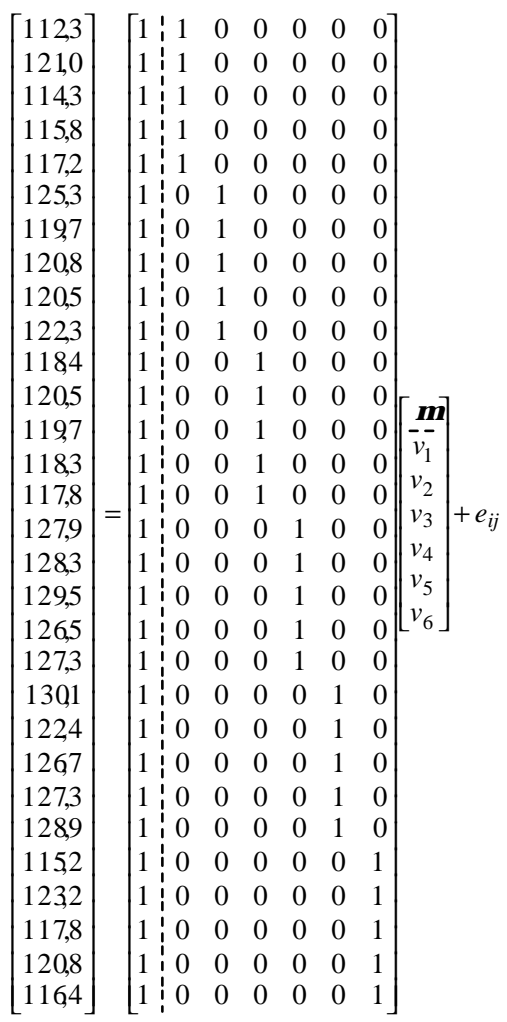


Na matriz $X$, a primeira coluna representa o efeito da constante $(\mu)$, e as demais representam os efeitos de variedades $\left(v_{1}, \ldots, v_{6}\right)$.

Os quatro tipos de funções estimáveis fornecidas pelo PROC GLM do SAS são equivalentes devido ao balanceamento dos dados. Sendo assim, as hipóteses respectivas também são equivalentes, resultando em somas de quadrados iguais (Tabela 14).

Tabela 14. Funções estimáveis para variedades fornecidas pelo PROC GLM do SAS.

\begin{tabular}{lcc}
\hline & & Tipo I $\approx$ Tipo II $\approx$ Tipo III $\approx$ Tipo IV \\
\hline$\mu$ & 0 & 0 \\
Variedades & 1 & L2 \\
& 2 & L3 \\
& 3 & L4 \\
& 4 & L5 \\
& 5 & L6 \\
& 6 & - L2 - L3 - L4 - L5 - L6 \\
\hline
\end{tabular}

$\approx:$ equivalente.

Por meio das funções estimáveis pode-se reconhecer a hipótese que está sendo testada. Para tanto, atribuiu-se arbitrariamente neste estudo, valor 1 para o coeficiente L2 e zero para os demais, escrevendo-se a primeira linha da hipótese. Procedendo-se da mesma forma para os demais coeficientes, constrói-se a hipótese completa, que neste caso é composta por 5 linhas (5 graus de liberdade):

$$
H_{0}:\left\{\begin{array}{l}
\mu_{1} \cdot-\mu_{6}=0 \\
\mu_{2} \cdot-\mu_{6}=0 \\
\mu_{3}-\mu_{6}=0 \\
\mu_{4} \cdot-\mu_{6}=0 \\
\mu_{5} \cdot-\mu_{6}=0
\end{array}\right.
$$

Portanto, a análise de variância para essa hipótese (Tabela 15), tem a soma de quadrados de variedades associada a um teste de que as variedades não diferem entre si, conforme explicitado nessa hipótese. 
Tabela 15. Análise de variância para os dados da Tabela 5.

\begin{tabular}{lcrccc}
\hline V.C.M.* & \multicolumn{1}{c}{ G.L. } & \multicolumn{1}{c}{ S.Q. } & Q.M. & F & Pr > F \\
\hline Variedades & $(t-1)=5$ & $R(\tau \mid \mu)=576,248$ & 115,2496 & 18,42 & 0,0001 \\
Resíduo & $(n-t)=24$ & $\Delta=150,144$ & 6,2560 & - & - \\
Total & $(n-1)=29$ & $R(\mu, \tau)=726,392$ & - & - & - \\
\hline
\end{tabular}

*Variações consideradas no modelo; $\boldsymbol{\mu}$ : denota o efeito da constante; $\tau$ : denota o efeito dos tratamentos; $\Delta=R(\mu, \tau)-R(\tau \mid \mu) ; t$ : número de tratamentos; $n:$ número de observações.

Como o teste $F$ para variedades foi significativo $\left(\operatorname{Pr}<0,0001\right.$ ), rejeita-se $H_{0}$ e conclui-se que as variedades apresentam efeitos diferentes sobre a produtividade de cana-de-açúcar. Essa conclusão é muito geral e nada informa com relação à comparação específica entre tratamentos. Dessa forma, usualmente a análise de variância é completada com testes de comparações múltiplas, por exemplo.

Quando se aplica o teste $F$ numa análise de variância com mais de um grau de liberdade, obtêm-se informações gerais, relacionadas com o comportamento médio dos tratamentos. Para esse conjunto de dados, a análise fornecida pelo PROC GLM do SAS não respondeu ao pesquisador, se as variedades dos grupos CB e IAC diferem, que é de seu interesse inicial. Contudo, é comum o pesquisador ter interesse em comparações específicas entre os tratamentos.

Por essa razão, havendo planejamento de comparações objetivas, é possível fazer o desdobramento dos graus de liberdade de tratamentos para obter informações relacionadas com o comportamento de cada um dos componentes do desdobramento. Além disso, após a decomposição ortogonal dos graus de liberdade, pode-se aplicar o teste $F$ aos componentes do desdobramento. 


\subsubsection{Desdobramento dos graus de liberdade de variedades usando contrastes ortogonais}

Examinando-se a Tabela 5, considerando que as comparações que interessam ao experimentador são aqueles que comparam variedades $\mathrm{CB}$ vs IAC, foram testados os seguintes contrastes ortogonais:

$$
\begin{aligned}
& \mathrm{Y}_{\mathrm{G} 1 \mathrm{vs} \mathrm{G} 2}: 2 \mu_{1}+2 \mu_{2} \cdot-\mu_{3} \cdot-\mu_{4} \cdot-\mu_{5 \cdot}-\mu_{6} \cdot=0 \\
& \mathrm{Y}_{\mathrm{V} 1 \mathrm{vs} \mathrm{V} 2}: \mu_{1} \cdot-\mu_{2} .=0 \\
& \mathrm{Y}_{\mathrm{V} 3, \mathrm{~V} 4 \mathrm{vs} \mathrm{V}, \mathrm{V} 6}: \mu_{3} \cdot+\mu_{4} \cdot-\mu_{5 \cdot}-\mu_{6} .=0 \\
& \mathrm{Y}_{\mathrm{V} 3 \mathrm{vs} \mathrm{V} 4}: \mu_{3} \cdot-\mu_{4} .=0 \\
& \mathrm{Y}_{\mathrm{v} 5 \mathrm{vs} 66}: \mu_{5 .}-\mu_{6} .=0
\end{aligned}
$$

em que:

G1: grupo formado pelas variedades CB (V1 e V2);

G2: grupo formado pelas variedades IAC (V3 a V6).

Para o cálculo das somas de quadrados das hipóteses, esses contrastes foram usados na forma de vetores, como a seguir:

$$
\begin{aligned}
& \mathrm{Y}_{\mathrm{G} 1 \text { vs G2 }}:\left[\begin{array}{llrrrr}
2 & 2 & -1 & -1 & -1 & -1
\end{array}\right] \\
& \mathrm{Y}_{\mathrm{V} 1 \text { vs V2 }}:\left[\begin{array}{lrrrrr}
1 & -1 & 0 & 0 & 0 & 0
\end{array}\right] \\
& \mathrm{Y}_{\mathrm{V} 3, \mathrm{~V} 4 \text { vs V5, V6 }}:\left[\begin{array}{cccccc}
0 & 0 & 1 & 1 & -1 & -1
\end{array}\right] \\
& \mathrm{Y}_{\mathrm{V} 3 \text { vs V4 }}:\left[\begin{array}{lllrrr}
0 & 0 & 1 & -1 & 0 & 0
\end{array}\right] \\
& \mathrm{Y}_{\mathrm{V} 5 \text { vs V6 }}:\left[\begin{array}{llllll}
0 & 0 & 0 & 0 & 1 & -1
\end{array}\right]
\end{aligned}
$$

O teste para a diferença entre as variedades dentro do grupo 2 foi feito considerando-se conjuntamente os três últimos contrastes.

Apresenta-se na Tabela 16 a análise de variância com desdobramento dos graus de liberdade de tratamentos, fornecida pela função CONTRAST do PROC GLM. 
Tabela 16. Análise de variância com desdobramento dos graus de liberdade de variedades, fornecida pela função CONTRAST do PROC GLM.

\begin{tabular}{lcrrcc}
\hline \multicolumn{1}{c}{ CONTRASTE } & G.L & SQ do Contraste & QM & F & Pr > F \\
\hline Variedade & 5 & 576,248 & 115,249 & 18,42 & 0,0001 \\
Y $_{\text {G1 vs G2 }}$ & 1 & 119,286 & 119,286 & 19,07 & 0,0002 \\
YV1 vs V2 (dentro G1) & 1 & 78,400 & 78,400 & 12,53 & 0,0017 \\
YV3_V4 vs V5_V6 & 1 & 1,458 & 1,458 & 0,23 & 0,6336 \\
YV3 vs V4 & 1 & 200,704 & 200,704 & 32,08 & 0,0001 \\
YV5 vs V6 & 1 & 176,400 & 176,400 & 28,20 & 0,0001 \\
Y(dentro G2) & $(3)$ & $(378,562)$ & $(126,187)$ & $(20,17)$ & 0,0001 \\
Resíduo & 2 & 150,144 & 6,256 & - & - \\
Total & \multicolumn{2}{c}{ 726,392 } & - & - \\
G1: grupo 1 (variedades CB, V1 e V2); G2: grupo 2 (variedades IAC, V3 a V6); V1: variedade 1; V2: \\
variedade 2; V3: variedade 3; V4: variedade 4; V5: variedade 5; V6: variedade 6.
\end{tabular}

Da análise de variância com desdobramento (Tabela 16), concluiu-se que as variedades do grupo CB (G1) e do grupo IAC (G2) são diferentes. Verifica-se também diferença entre os efeitos das variedades 1 e 2, assim como diferença entre os efeitos das variedades 3 e 4 e das variedades 5 e 6 . Essas informações são fundamentais para interpretação dos resultados e não são obtidas por meio da análise de variância convencional, sendo o teste de contrastes ortogonais uma das formas de se resolver essas deficiências em informações. Efetivamente, as hipóteses de interesse do pesquisador, que também consideram saber se as diferenças puderam ser verificadas entre as variedades dentro de cada grupo, são obtidos com a função CONTRAST (Tabela 16). No caso, conclui-se que as variedades dentro do grupo 1 diferem, assim como as variedades dentro do grupo 2. A função CONTRAST do PROC GLM do SAS é de fácil utilização, entretanto, em experimentos com um grande número de tratamentos, a construção de contrastes torna-se trabalhosa. No entanto, permitiu testar hipóteses de interesse. 
É importante destacar que uma hipótese que o pesquisador buscou testar nesse experimento refere-se à diferença entre as variedades do grupo $\mathrm{CB}$ vs IAC. Isso foi testado no primeiro contraste, que apresenta apenas 1 grau de liberdade pois há apenas dois grupos. As diferenças dentro do grupo 1 foram testadas no contraste $Y_{V 1}$ vs v2 e as dentro do grupo IAC, pelo teste conjunto dos contrastes $\mathrm{Y}_{\mathrm{V} 3}$, v4 vs v5, v6; $\mathrm{Y}_{\mathrm{V} 3 \text {, vs V4 }}$ e $\mathrm{Y}_{\mathrm{V} 5 \text { vs }}$ v6.

\subsubsection{Análise de variância pela técnica das variáveis auxiliares}

Uma alternativa mais simples ao uso de contrastes é aplicar a técnica das variáveis auxiliares. Essa técnica permite a obtenção de informações mais específicas, relacionadas com o comportamento dos componentes do desdobramento proposto. Assim, o experimento da Tabela 5 foi dividido em 2 grupos de interesse, utilizando as variáveis auxiliares $C$ e $D$. A variável auxiliar $C$ denota o efeito das variedades 1 e 2 (grupo de variedades CB), e a variável auxiliar $D$ denota o efeito das variedades 3, 4, 5 e 6 (grupo de variedades IAC). Adotou-se o modelo linear superparametrizado $(y=X \boldsymbol{\theta}+e)$, agora caracterizado por $y_{i j}=\boldsymbol{\mu}+d_{i}+c_{i}+e_{i j}$. Assim, para essa caracterização, obteve-se matricialmente: 


\begin{tabular}{|c|c|c|c|c|c|c|c|c|}
\hline$[112,3]$ & {$\left[\begin{array}{l:l}1 & 1\end{array}\right.$} & 0 & 0 & 0 & $0: 1$ & & & \\
\hline 121,0 & $1: 1$ & 0 & 0 & 0 & $0: 1$ & 0 & 0 & \\
\hline 114,3 & $1: 1$ & 0 & 0 & 0 & $\begin{array}{l:l}0 & 1\end{array}$ & 0 & 0 & \\
\hline 115,8 & $\begin{array}{l:ll}1 & 1\end{array}$ & 0 & 0 & 0 & $0: \begin{array}{l:}0 \\
0\end{array}$ & 0 & 0 & \\
\hline 117,2 & $1: 1$ & 0 & 0 & 0 & $0: 1$ & 0 & 0 & \\
\hline 125,3 & $1: 1$ & 0 & 0 & 0 & 0 & 1 & 0 & \\
\hline 119,7 & $\begin{array}{l:l}1 & 1\end{array}$ & 0 & 0 & 0 & $0: 0$ & 1 & 0 & \\
\hline 120,8 & $\begin{array}{l:l}1 & 1\end{array}$ & 0 & 0 & 0 & $\begin{array}{l:l}0 & 0\end{array}$ & 1 & 0 & \\
\hline 120,5 & $1: 1$ & 0 & 0 & 0 & $0: 0$ & 1 & 0 & \\
\hline 122,3 & $1: 1$ & 0 & 0 & 0 & $0: 0$ & 1 & 0 & \\
\hline 118,4 & $\begin{array}{l:l}1 & 0\end{array}$ & 1 & 0 & 0 & 0 & 0 & 1 & \\
\hline 120,5 & $1: 0$ & 1 & 0 & 0 & 0 & 0 & 1 & \\
\hline 119,7 & $\begin{array}{l:l}1 & 0\end{array}$ & 1 & 0 & 0 & 0 & 0 & 1 & \\
\hline 118,3 & $1: 0$ & 1 & 0 & 0 & 0 & 0 & 1 & \\
\hline 117,8 & $1: 0$ & 1 & 0 & 0 & $0: 0$ & 0 & 1 & \\
\hline 127,9 & $\begin{array}{l:l}1 & 0\end{array}$ & 0 & 1 & 0 & 0 & 0 & 1 & \\
\hline 128,3 & $\begin{array}{l:l}1 & 0\end{array}$ & 0 & 1 & 0 & 0 & 0 & 1 & \\
\hline 129,5 & $1: 0$ & 0 & 1 & 0 & $0: 0$ & 0 & 1 & \\
\hline 126,5 & $\begin{array}{l:ll}1 & 0\end{array}$ & 0 & 1 & 0 & $0: 0$ & 0 & 1 & \\
\hline 127,3 & $1: 0$ & 0 & 1 & 0 & $0: 0$ & 0 & 1 & \\
\hline 130,1 & $1: 0$ & 0 & 0 & 1 & $0: 0$ & 0 & 1 & \\
\hline 1224 & $\begin{array}{l:l}1 & 0\end{array}$ & 0 & 0 & 1 & $0: 0$ & 0 & 1 & \\
\hline 126,7 & 10 & 0 & 0 & 1 & 0 & 0 & 1 & \\
\hline 127,3 & \begin{tabular}{l|l}
1 & 0
\end{tabular} & 0 & 0 & 1 & $0: 0$ & 0 & 1 & \\
\hline 128,9 & $\begin{array}{l:ll}1 & 0\end{array}$ & 0 & 0 & 1 & 0 & 0 & 1 & \\
\hline 115,2 & $1: 0$ & 0 & 0 & 0 & $1: c$ & 0 & 1 & \\
\hline 123,2 & $\begin{array}{l:l}1 & 0\end{array}$ & 0 & 0 & 0 & 1 & 0 & 1 & \\
\hline 117,8 & $\begin{array}{l:l}1 & 0\end{array}$ & 0 & 0 & 0 & 1 & 0 & 1 & \\
\hline 120,8 & $1: 0$ & 0 & 0 & 0 & $1:$ & 0 & 1 & \\
\hline 116,4 & $\begin{array}{l:ll}1 & 0\end{array}$ & 0 & 0 & 0 & $1:$ & ( & 1 & \\
\hline
\end{tabular}

Na matriz $X$, a primeira coluna denota o efeito da constante $(\boldsymbol{\mu})$, as colunas 2 , 3, 4, 5 e 6 denotam os efeitos das variedades assumidas por $D$ e as três últimas denotam os efeitos das variedades assumidas por $C$. Tais efeitos correspondem ao vetor de parâmetros desconhecidos $\theta$.

Os índices dizem respeito aos níveis de $D$ e $C$ :

$d_{0}$ : nível da variável auxiliar $D$ que assume valor 1 para as variedades 1 e 2 (grupo 1) e 0 caso contrário.

$d_{3}$ : nível da variável auxiliar $D$ que assume valor 1 para a variedade 3 e 0 caso contrário. 
$d_{4}$ : nível da variável auxiliar $D$ que assume valor 1 para a variedade 4 e 0 caso contrário.

$d_{5}$ : nível da variável auxiliar $D$ que assume valor 1 para a variedade 5 e 0 caso contrário.

$d_{6}$ : nível da variável auxiliar $D$ que assume valor 1 para a variedade 6 e 0 caso contrário.

$c_{0}$ : nível da variável auxiliar $C$ que assume valor 1 para as variedades 3 a 6 (grupo 2) e 0 caso contrário.

$c_{1}$ : nível da variável auxiliar $C$ que assume valor 1 para a variedade 1 e 0 caso contrário.

$c_{2}$ : nível da variável auxiliar $C$ que assume valor 1 para a variedade 2 e 0 caso contrário.

As funções estimáveis para esse modelo e conjunto de dados, fornecidas pelo PROC GLM do SAS, estão apresentadas na Tabela 17.

Tabela 17. Funções estimáveis para variáveis auxiliares fornecidas pelo PROC GLM do SAS.

\begin{tabular}{cccccc}
\hline & \multicolumn{2}{c}{ Fator $C$} & \multicolumn{2}{c}{ Fator $D$} \\
& & Tipo I & Tipo II = III = IV & Tipo I & Tipo II = III = IV \\
\hline$\mu$ & & 0 & 0 & 0 & 0 \\
$C$ & 0 & L2 & 0 & 0 & 0 \\
& 1 & L3 & L3 & 0 & 0 \\
& 2 & -L2-L3 & -L3 & 0 & 0 \\
& & & & & 0 \\
& 0 & - L2 & 0 & 0 & L6 \\
& 3 & 0,25 L2 & 0 & L6 & L7 \\
& 4 & 0,25 L2 & 0 & L8 & L8 \\
& 5 & 0,25 L2 & 0 & -L6-L7-L8 & -L6-L7-L8 \\
\hline
\end{tabular}


Pela Tabela 17, para o fator $C$ a função estimável do tipo I é diferente das demais, o que resultará em uma soma de quadrados diferente. Nesse caso, a hipótese do tipo I não é de interesse prático para o pesquisador. Entretanto, fazendo-se L2 $=0$ e L3 = 1, tem-se uma hipótese equivalente a do tipo III. As funções estimáveis dos tipos II, III e IV são equivalentes, e por isso têm um mesmo valor para soma de quadrados.

Atribuindo-se, valor 1 para o coeficiente L3, a hipótese que está sendo testada é dada por:

$$
H_{0}: \mu_{1}-\mu_{2}=0
$$

Logo, a soma de quadrados para $C$ (Tabela 18), está associada a um teste de hipótese de que a média da variedade 1 não difere da variedade 2, ou seja:

$$
\text { Variedade } 1 \text { vs. Variedade } 2 \text { (1 g.1.) }
$$

É fácil verificar que essa hipótese é a mesma já testada na Tabela 16.

A hipótese para o fator $D$ foi escrita atribuindo-se valor 1 para o coeficiente L6 e zero para os demais, construindo-se a primeira linha da hipótese. Procedendo da mesma forma para os demais coeficientes tem-se a hipótese:

$$
H_{0}:\left\{\begin{array}{l}
\mu_{3}-\mu_{6}=0 \\
\mu_{4 \cdot}-\mu_{6}=0 \\
\mu_{5 \cdot}-\mu_{6}=0
\end{array}\right.
$$

O teste da hipótese para $D$ (Tabela 18), verifica se há diferença entre as médias das variedades do grupo 2. Isso é equivalente a desdobrar os componentes de $D$ nos contrastes:

Variedade 3 vs. Variedade 6 (1 g.1.)

Variedade 4 vs. Variedade 6 (1 g.l.)

Variedade 5 vs. Variedade 6 (1 g.l.) 
É interessante notar que não há nenhuma comparação envolvendo $c_{0} \mathrm{e} d_{0}$, conforme desejado

$\mathrm{Na}$ análise de variância (Tabela 18), percebe-se que os 5 graus de liberdade para variedades estão decompostos em 1 grau de liberdade para os níveis de $C$ (grupo com 2 variedades) e 3 graus de liberdade para os níveis de $D$ (grupo com 4 variedades). Em relação à Tabela 15, não é testado com 1 grau de liberdade, o contraste entre grupos $1 \mathrm{e}$ 2.

Tabela 18. Análise de variância obtida com o uso de variável auxiliar.

\begin{tabular}{lccccc}
\hline V.C.M. & G.L. & S.Q. & Q.M. & F & Pr $>$ F \\
\hline$C$ & $\left(c^{\prime}-1\right)=1$ & $R(c \mid \mu, d)=78,4000$ & 78,4000 & 12,53 & 0,0001 \\
$D$ & $\left(d^{\prime}-1\right)=3$ & $R(d \mid \mu, c)=378,5620$ & 126,1873 & 20,17 & 0,0001 \\
Resíduo & $(n-1)=24$ & $R(\mu, \tau)-R(\tau \mid \mu)=150,1440$ & 6,2560 & 20,17 & 0,0001 \\
Total & $(n-1)=29$ & $R(\mu, \tau)=726,3920$ & - & - & - \\
\hline$\mu:$ efeito da constante; $C:$ efeito das variedades do grupo $1 ; D:$ efeito das variedades do grupo $2 ; c^{\prime}:$ \\
número de níveis de $C$, exceto $c_{0} ; d^{\prime}:$ número de níveis de $D$, exceto $d_{0}$. & &
\end{tabular}

Observando a Tabela 18, tem-se o teste F significativo para $C$ e para $D$. Assim, conclui-se que os efeitos sobre a produtividade das variedades 1 e 2 são diferentes, bem como pelo menos duas das variedades do grupo 2 (variedades $3,4,5$ e 6 ).

Essa decomposição em contrastes ortogonais, foi testada equivalentemente pela função CONTRAST do PROC GLM (Tabela 16). Entretanto, a realização dessa análise torna-se mais simples com o uso de variáveis auxiliares, pois não é necessário que o pesquisador construa os contrastes ortogonais.

Utilizando-se a técnica da variável auxiliar realiza-se uma análise de variância mais diretamente, tendo em vista que os tratamentos são previamente divididos de acordo com os grupos de interesse. Além disso, em situações reais, com número elevado de tratamentos, o número de contrastes a ser construído é muito grande. 
No entanto, para a situação aqui descrita, após o uso da técnica das variáveis auxiliares ainda é necessária a aplicação de um teste para detectar se há diferenças entre os grupos 1 e 2 . Mesmo assim, será necessário construir contrastes somente entre os grupos pré-estabelecidos pelo pesquisador.

\subsection{Conjunto B}

\subsubsection{Hipóteses testadas automaticamente pelo PROC GLM do SAS}

Para o experimento apresentado na Tabela 6, adotou-se a caracterização $y_{i j}=\mu+\beta_{j}+\tau_{i}+e_{i j}$, tendo o modelo linear a seguinte representação matricial $(y=X \boldsymbol{\theta}+e)$

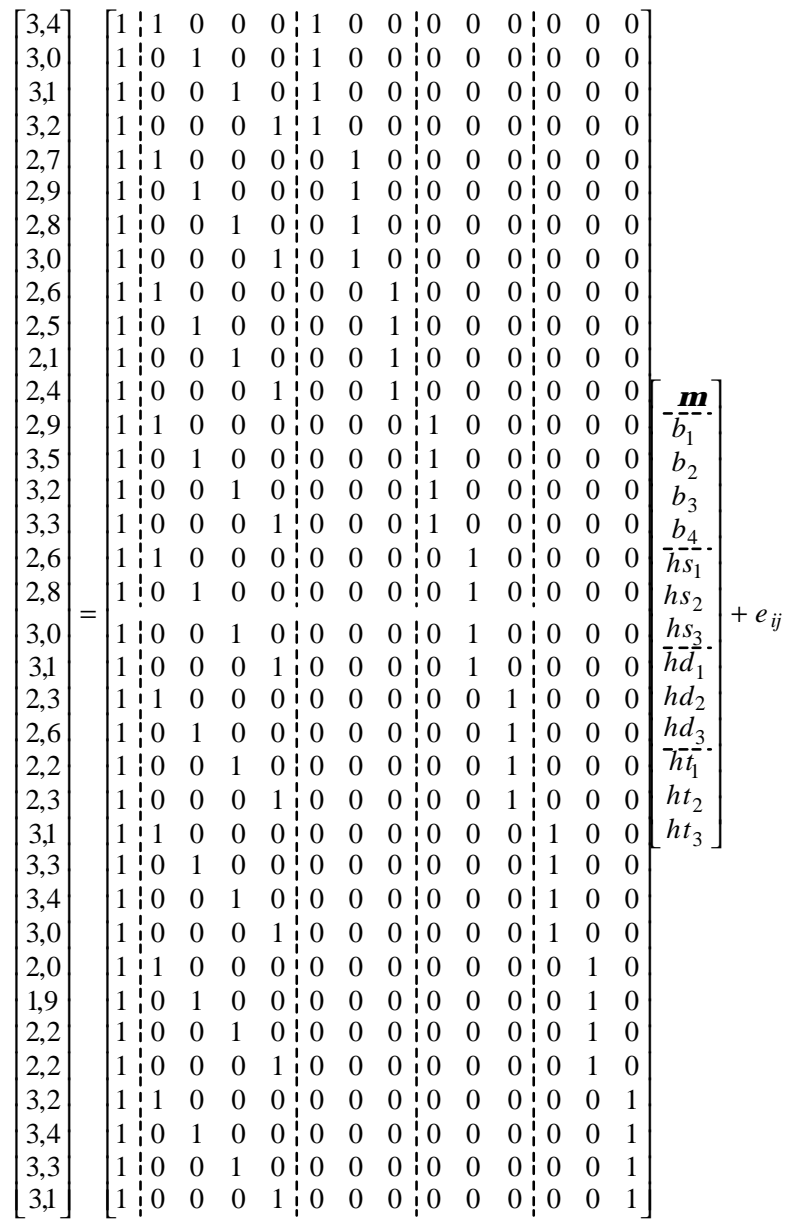


Na matriz $X$, a primeira coluna denota o efeito da constante $(\boldsymbol{\mu})$, as colunas 2 a 5 representam os efeitos de blocos, e as demais referem-se aos efeitos de híbridos simples (HS), duplos (HD) e triplos (HT), respectivamente.

Tendo em vista que os dados são balanceados, os quatro tipos de funções estimáveis para híbridos são equivalentes (Tabela 19), o que resultou em um mesmo valor de soma de quadrados (Tabela 20), para os quatro tipos de funções estimáveis.

Tabela 19. Funções estimáveis para híbridos fornecidas pelo PROC GLM do SAS.

\begin{tabular}{lcc}
\hline & & Tipo I $\approx$ Tipo II $\approx$ Tipo III $\approx$ Tipo IV \\
\hline Blocos & 0 & 0 \\
& 1 & 0 \\
& 2 & 0 \\
& 3 & 0 \\
Híbridos & 4 & 0 \\
& 1 & \\
& 2 & L6 \\
& 3 & L7 \\
& 4 & L8 \\
& 5 & L9 \\
& 6 & L10 \\
& 7 & L11 \\
& 8 & L12 \\
& 9 & L13 \\
& &
\end{tabular}

$\approx:$ equivalentes.

Para construção da hipótese, atribuiu-se inicialmente valor 1 para o coeficiente L6 e zero para os demais, escrevendo-se a primeira linha da hipótese. Procedendo da mesma forma para os demais coeficientes, constrói-se a hipótese para híbridos, com 8 graus de liberdade: 


$$
H_{0}:\left\{\begin{array}{l}
\mu_{1 \cdot}-\mu_{9}=0 \\
\mu_{2} \cdot-\mu_{9}=0 \\
\mu_{3}-\mu_{9}=0 \\
\mu_{4}-\mu_{9}=0 \\
\mu_{5 \cdot}-\mu_{9}=0 \\
\mu_{6} \cdot-\mu_{9}=0 \\
\mu_{7}-\mu_{9}=0 \\
\mu_{8}-\mu_{9}=0
\end{array}\right.
$$

Essa hipótese está associada a um teste de que as médias de produtividade dos nove híbridos não diferem entre si. A análise de variância respectiva é apresentada na Tabela 20.

Tabela 20. Análise de variância para os dados da Tabela 6.

\begin{tabular}{|c|c|c|c|c|c|}
\hline V.C.M. & G.L. & S.Q. & Q.M. & $\mathrm{F}$ & $\operatorname{Pr}>F$ \\
\hline Blocos & $(b-1)=3$ & $R(\beta \mid \mu, \tau)=0,0733$ & 0,0244 & 0,69 & 0,5654 \\
\hline Híbridos & $(t-1)=8$ & $R(\tau \mid \mu, \beta)=6,3023$ & 0,7878 & 22,33 & 0,0001 \\
\hline Resíduo & $(n-t)=24$ & $\Delta=0,8467$ & 0,0353 & - & - \\
\hline Total & $(n-1)=35$ & $R(\mu, \beta, \tau)=7,2222$ & - & - & - \\
\hline
\end{tabular}

Como o teste $F$ para híbridos é significativo ( $\operatorname{Pr}<0,0001$ ), rejeita-se $H_{0}$ e conclui-se que os híbridos possuem efeitos diferentes sobre a produtividade. Essa conclusão, por ser muito geral, nada informa com relação à comparação entre os híbridos de cada tipo (simples, duplos e triplos). Para obter essas informações, pode-se realizar um teste de comparação de médias, ou ainda decompor ortogonalmente os graus de liberdade para tratamentos. Na escolha dos componentes do desdobramento, deve-se levar em consideração as comparações que interessam ao pesquisador. Dessa forma, da mesma maneira que para o conjunto $\mathrm{A}$, não foi possível responder diretamente a questão de interesse do pesquisador. 


\subsubsection{Desdobramento dos graus de liberdade de híbridos usando contrastes ortogonais}

Desejando-se obter comparações de interesse, pode-se construir um conjunto de contrastes ortogonais e testá-los por meio da função CONTRAST do PROC GLM. Esse é um teste complementar ao teste F, que auxilia na interpretação dos resultados, completando a análise.

Para o conjunto de dados $\mathrm{B}$, determinaram-se comparações que podem interessar ao experimentador. Os componentes do desdobramento proposto com os respectivos contrastes são dados a seguir:

$$
\begin{aligned}
& \mathrm{Y}_{\mathrm{HS} \text { vs HD, HT }}: 2 \mu_{1} \cdot+2 \mu_{2} \cdot+2 \mu_{3 .}-\mu_{4} \cdot-\mu_{5 \cdot}-\mu_{6 \cdot}-\mu_{7 \cdot}-\mu_{8} \cdot-\mu_{9 \cdot}=0 \\
& \mathrm{Y}_{\mathrm{HS} 1 \mathrm{vs} \mathrm{HS} 2, \mathrm{HS} 3}: 2 \mu_{1} \cdot-\mu_{2} \cdot-\mu_{3} .=0 \\
& \mathrm{Y}_{\mathrm{HS} 2 \mathrm{vs} \mathrm{HS} 3}: \mu_{2} \cdot-\mu_{3}=0 \\
& \mathrm{Y}_{\mathrm{HD} \mathrm{vs} \mathrm{HT}}: \mu_{4} \cdot+\mu_{5} \cdot+\mu_{6} \cdot-\mu_{7} \cdot-\mu_{8} \cdot-\mu_{9}=0 \\
& \mathrm{Y}_{\mathrm{HD} 4 \mathrm{vs} \mathrm{HD} \text {, HD6 }}: 2 \mu_{4} \cdot-\mu_{5 \cdot}-\mu_{6} .=0 \\
& \mathrm{Y}_{\mathrm{HD} 5 \mathrm{vs} \mathrm{HD6}}: \mu_{5} \cdot-\mu_{6} \cdot=0
\end{aligned}
$$

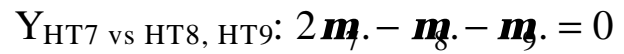

$$
\begin{aligned}
& \mathrm{Y}_{\mathrm{HT} 8 \mathrm{vs} \mathrm{HT} 9}: \mu_{8} \cdot-\mu_{9} .=0
\end{aligned}
$$

em que:

G1: grupo formado pelos híbridos simples (HS1, HS2 e HS3);

G2: grupo formado pelos híbridos duplos (HD1, HD2 e HD3);

G2: grupo formado pelos híbridos triplos (HT1, HT2 e HT3).

Para o cálculo das somas de quadrados das hipóteses, esses contrastes foram usados na forma de vetores, como a seguir:

$$
\begin{aligned}
& \mathrm{Y}_{\mathrm{HS} \text { vs HD, HT }}:\left[\begin{array}{lllllllll}
2 & 2 & 2 & -1 & -1 & -1 & -1 & -1 & -1
\end{array}\right] \\
& \mathrm{Y}_{\mathrm{HS} 1 \mathrm{vs} \mathrm{HS} 2, \mathrm{HS} 3}:\left[\begin{array}{lllllllll}
2 & -1 & -1 & 0 & 0 & 0 & 0 & 0 & 0
\end{array}\right]
\end{aligned}
$$




$$
\begin{aligned}
& \mathrm{Y}_{\mathrm{HS} 2 \text { vs HS3 }}:\left[\begin{array}{lllllllll}
0 & 1 & -1 & 0 & 0 & 0 & 0 & 0 & 0
\end{array}\right] \\
& \mathrm{Y}_{\mathrm{HD} \text { vs HT }}:\left[\begin{array}{llllllllll}
0 & 0 & 0 & 1 & 1 & 1 & -1 & -1 & -1
\end{array}\right] \\
& \mathrm{Y}_{\mathrm{HD} 4 \text { vs HD5, HD6 }}:\left[\begin{array}{lllllllll}
0 & 0 & 0 & 2 & -1 & -1 & 0 & 0 & 0
\end{array}\right] \\
& \mathrm{Y}_{\mathrm{HD} 5 \text { vs HD6 }}:\left[\begin{array}{lllllllll}
0 & 0 & 0 & 0 & 1 & -1 & 0 & 0 & 0
\end{array}\right] \\
& \mathrm{Y}_{\mathrm{HT7} \text { vs HT8, HT9: }}\left[\begin{array}{lllllllll}
0 & 0 & 0 & 0 & 0 & 0 & 2 & -1 & -1
\end{array}\right] \\
& \mathrm{Y}_{\mathrm{HT} 8 \text { vs HT9: }}\left[\begin{array}{lllllllll}
0 & 0 & 0 & 0 & 0 & 0 & 0 & 1 & -1
\end{array}\right]
\end{aligned}
$$

Para testar se há diferença entre os híbridos dentro dos híbridos simples, foi construído um contraste com 2 graus de liberdade, usando os coeficientes dos contrastes

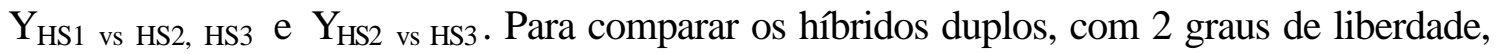
usou-se os coeficientes de $\mathrm{Y}_{\mathrm{HD} 4}$ vs HD5, HD6 e $\mathrm{Y}_{\mathrm{HD}}$ vs HD6. Analogamente, para os híbridos

\begin{tabular}{|c|c|c|c|c|c|}
\hline Contraste & GL & SQ dos Contrastes & QM & $\mathrm{F}$ & $\operatorname{Pr}>F$ \\
\hline Blocos & 3 & 0,0733 & 0,0244 & 0,69 & 0,5654 \\
\hline Híbridos & 8 & 6,3023 & 0,7878 & 22,33 & 0,0001 \\
\hline$Y_{\text {HS vs HD_HT }}$ & 1 & 0,0035 & 0,0035 & 0,10 & 0,7564 \\
\hline $\mathrm{Y}_{\mathrm{H} 1 \mathrm{vs} \mathrm{H} 2 \_\mathrm{H} 3}$ & 1 & 0,8067 & 0,8067 & 22,87 & 0,0001 \\
\hline $\mathrm{Y}_{\mathrm{H} 2 \mathrm{vs} \mathrm{H} 3}$ & 1 & 0,4050 & 0,4050 & 11,48 & 0,0024 \\
\hline (Y dentro de HS) & (2) & $(1,2117)$ & $(0,6058)$ & $(17,16)$ & $(0,0001)$ \\
\hline $\mathrm{Y}_{\mathrm{G} 2 \text { vs G3 }}$ & 1 & 0,0037 & 0,0037 & 0,11 & 0,7472 \\
\hline $\mathrm{Y}_{\mathrm{H} 4 \mathrm{vs} \mathrm{H} 5 \_\mathrm{H} 6}$ & 1 & 1,0004 & 1,0004 & 28,36 & 0,0001 \\
\hline $\mathrm{Y}_{\mathrm{H} 5 \mathrm{vs} \mathrm{H} 6}$ & 1 & 0,5512 & 0,5512 & 15,63 & 0,0006 \\
\hline (Y dentro de HD) & (2) & $(1,5516)$ & $(0,7758)$ & $(21,98)$ & $(0,0001)$ \\
\hline $\mathrm{Y}_{\mathrm{H} 7 \mathrm{vs} \mathrm{H} 8 \_\mathrm{H} 9}$ & 1 & 0,7704 & 0,7704 & 21,84 & 0,0001 \\
\hline $\mathrm{Y}_{\mathrm{H} 8 \mathrm{vs} \mathrm{H} 9}$ & 1 & 2,7612 & 2,7612 & 78,27 & 0,0001 \\
\hline (Y dentro de HT) & (2) & $(3,5316)$ & $(1,7658)$ & $(50,02)$ & $(0,0001)$ \\
\hline Resíduo & 24 & 0,8467 & 0,0353 & - & - \\
\hline Total & 35 & 7,2220 & 0,2063 & - & - \\
\hline
\end{tabular}
triplos, usou-se $\mathrm{Y}_{\mathrm{HT} 7 \text { vs HT8, HT9 }}$ e $\mathrm{Y}_{\mathrm{HT}}$ vs HT9, com 2 graus de liberdade.

$\mathrm{Na}$ Tabela 21 são apresentadas as análises de variâncias com desdobramentos dos graus de liberdade para híbridos, fornecidas pela Função CONTRAST do PROC GLM.

Tabela 21. Análise de variância com desdobramento dos graus de liberdade de híbridos, fornecida pela função CONTRAST do PROC GLM. 
HS: híbridos simples; HD: híbridos duplos; HT: híbridos triplos; H1: híbrido simples 1; H2: híbrido simples 2; H3: híbrido simples 3; H4: híbrido duplo 1; H5: híbrido duplo 2; H6: híbrido duplo 3; H7: híbrido triplo 1; H8: híbrido triplo 2; H9: híbrido triplo 3.

Observando-se a Tabela 21, conclui-se que os grupos de híbridos não diferem entre si, entretanto há diferenças entre os efeitos sobre a produtividade dos híbridos dentro de cada grupo, ou seja, há diferenças entre os híbridos dentro do grupo híbridos simples, dentro do grupo híbridos duplos e dentro do grupo híbridos triplos. Por exemplo, o híbrido simples 1 difere dos híbridos simples 2 e 3, assim como o híbrido simples 2 difere do híbrido simples 3. Além disso, o HD 4 difere dos HD 5 e 6 e HD 5 difere do HD 6. Há diferença também entre todos os híbridos triplos, ou seja, os híbridos 7, 8 e 9 diferem entre si.

Realizar a análise dessa forma requer a construção de todos os contrastes de interesse e, por meio de suas funções paramétricas, testá-los utilizando a função CONTRAST. Este procedimento é simples para experimento pequenos, com poucos tratamentos, mas torna-se trabalhoso para um grande número de tratamentos. Assim, nota-se que, embora seja possível testar as hipóteses de interesse, muitas vezes essa tarefa é trabalhosa.

\subsubsection{Análise de variância pela técnica das variáveis auxiliares}

Uma alternativa à análise de variância convencional, com aplicação simples, é o uso da técnica das variáveis auxiliares, associada à função CONTRAST.

Para o conjunto de dados B (Tabela 6), definiram-se as variáveis auxiliares $C$, $D$ e $F$. Reescrevendo-se a caracterização do modelo linear usou-se $y_{i j}=\boldsymbol{\mu}+\boldsymbol{\beta}_{j}+c_{i}+d_{i}+f_{i}+e_{i j}$.

Matricialmente obteve-se: 


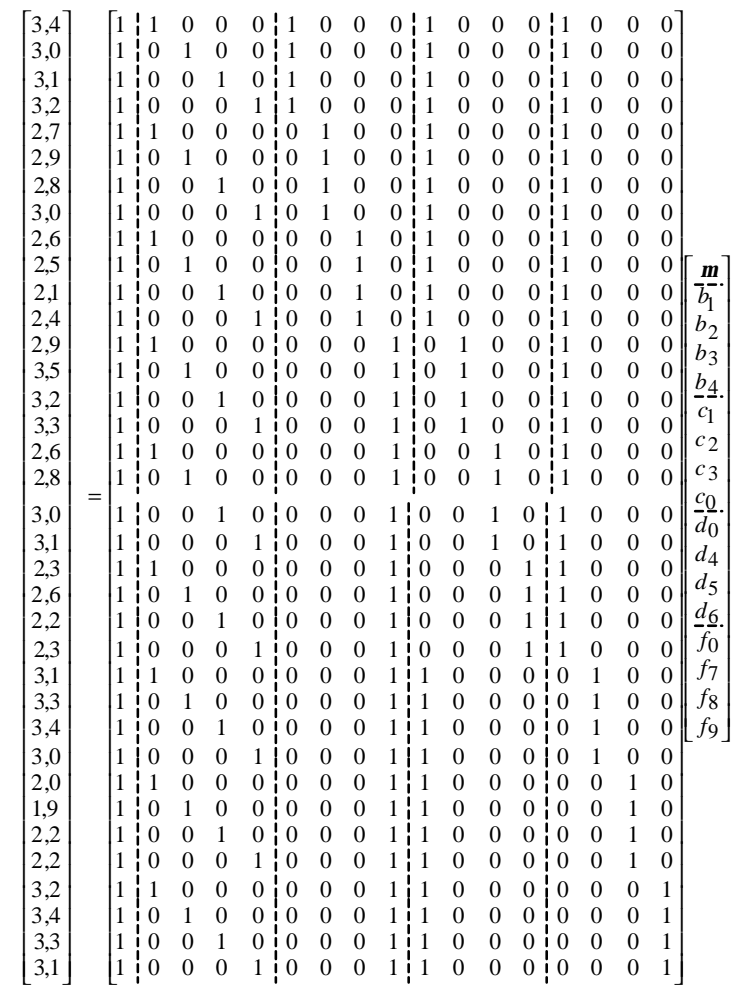

Assim, a matriz do delineamento foi formada pela coluna da constante $(\boldsymbol{\mu})$, seguida pelas colunas que denotam o efeito de blocos $(b)$, e pelas colunas que denotam os efeitos de $C, D$ e $F$. Os índices dizem respeito aos níveis:

$b_{1}$ : efeito do bloco 1 ;

$b_{2}$ : efeito do bloco 2 ;

$b_{3}$ : efeito do bloco 3 ;

$b_{4}$ : efeito do bloco 4 ;

$c_{1}$ : nível da variável auxiliar $C$, que assume valor 1 para o híbrido simples 1 e zero em caso contrário;

$c_{2}$ : nível da variável auxiliar $C$, que assume valor 1 para o híbrido simples 2 e zero em caso contrário;

$c_{3}$ : nível da variável auxiliar $C$, que assume valor 1 para o híbrido simples 3 e zero em caso contrário; 
$c_{0}$ : nível da variável auxiliar $C$ que assume o valor zero para os híbridos simples e 1 nos casos contrários;

$d_{0}$ : variável auxiliar $D$ que assume o valor zero para os híbridos duplos e 1 nos caso contrários;

$d_{4}$ : nível da variável auxiliar $D$, que assume o valor 1 para o híbrido duplo 1 e zero em caso contrário;

$d_{5}$ : nível da variável auxiliar $D$ que assume valor 1 para o híbrido duplo 2 e zero em caso contrário;

$d_{6}$ : nível da variável auxiliar $D$, que assume valor 1 para o híbrido duplo 3 e zero em caso contrário;

$f_{0}$ : nível da variável auxiliar $F$, que assume o valor zero para os híbridos triplos e 1 em caso contrário;

$f_{1}$ : nível da variável auxiliar $F$, que assume o valor 1 para o híbrido triplo 1 e zero em caso contrário;

$f_{2}$ : nível da variável auxiliar $F$, que assume o valor 1 para o híbrido triplo 2 e zero em caso contrário;

$f_{3}$ : nível da variável auxiliar $F$, que assume o valor 1 para o híbrido triplo 3 e zero em caso contrário.

Para esse conjunto de dados e caracterização do modelo linear adotada, somente as funções estimáveis dos tipos II, III e IV são equivalentes (Tabela 22), resultando em somas de quadrados iguais (Tabela 23). A função estimável do tipo I não é equivalente às demais, resultando em uma soma de quadrados diferente. 
Tabela 22. Funções estimáveis para as variáveis auxiliares $C, D e F$.

\begin{tabular}{|c|c|c|c|c|c|c|c|}
\hline & \multicolumn{3}{|c|}{ Tipo I } & \multicolumn{3}{|c|}{ Tipo II = Tipo III = Tipo IV } \\
\hline & & Fator $C$ & Fator $D$ & Fator $F$ & Fator $C$ & Fator $D$ & Fator $F$ \\
\hline$\mu$ & & 0 & 0 & 0 & 0 & 0 & 0 \\
\hline \multirow[t]{4}{*}{ Blocos } & 1 & 0 & 0 & 0 & 0 & 0 & 0 \\
\hline & 2 & 0 & 0 & 0 & 0 & 0 & 0 \\
\hline & 3 & 0 & 0 & 0 & 0 & 0 & 0 \\
\hline & 4 & 0 & 0 & 0 & 0 & 0 & 0 \\
\hline \multirow[t]{4}{*}{$C$} & 0 & L6 & 0 & 0 & 0 & 0 & 0 \\
\hline & 1 & L7 & 0 & 0 & L7 & 0 & 0 \\
\hline & 2 & L8 & 0 & 0 & L8 & 0 & 0 \\
\hline & 3 & -L6-L7-L8 & 0 & 0 & -L7-L8 & 0 & 0 \\
\hline \multirow[t]{4}{*}{$D$} & 0 & $-0,5 \mathrm{~L} 6$ & L10 & 0 & 0 & 0 & 0 \\
\hline & 4 & $0,167 \mathrm{~L} 6$ & L11 & 0 & 0 & L11 & 0 \\
\hline & 5 & $0,167 \mathrm{~L} 6$ & L12 & 0 & 0 & L12 & 0 \\
\hline & 6 & $0,167 \mathrm{~L} 6$ & -L10-L11-L12 & 0 & 0 & -L11-L12 & 0 \\
\hline \multirow[t]{4}{*}{$F$} & 0 & $-0,5 \mathrm{~L} 6$ & -L10 & 0 & 0 & 0 & 0 \\
\hline & 7 & $0,167 \mathrm{~L} 6$ & $0,333 \mathrm{~L} 10$ & L15 & 0 & 0 & L15 \\
\hline & 8 & $0,167 \mathrm{~L} 6$ & 0,333L10 & L16 & 0 & 0 & L16 \\
\hline & 9 & $0,167 \mathrm{~L} 6$ & $0,333 \mathrm{~L} 10$ & -L15-L16 & 0 & 0 & -L15-L16 \\
\hline
\end{tabular}

A hipótese do tipo I é de interesse do pesquisador somente para o fator F. Dessa forma, serão utilizadas as funções estimáveis dos tipos II, III, IV, que são de interesse para todos os fatores.

Para o fator $C$, a primeira linha da hipótese é escrita, atribuindo-se valor 1 para o coeficiente L7 e zero para o coeficiente L8. Em seguida, atribui-se valor 1 para o coeficiente L8 e zero para o coeficiente L7, obtendo a segunda linha da hipótese:

$$
H_{0}:\left\{\begin{array}{l}
\mu_{1} \cdot-\mu_{3}=0 \\
\mu_{2} \cdot-\mu_{3}=0
\end{array}\right.
$$

A soma de quadrados para essa hipótese está associada a um teste de que as médias de produtividade dos híbridos simples não diferem entre si. Essa hipótese pode ser entendida, alternativamente, como a decomposição a seguir conjuntamente:

Híbrido 1 vs. Híbrido 2, Híbrido 3 (1 g.l.) 
Híbrido 2 vs. Híbrido 3 (1 g.l.)

Tal decomposição, testada com a função CONTRAST, é idêntica a que foi obtida com a variável auxiliar.

As hipóteses para os fatores $D$ e $F$ são construídas atribuindo-se valor 1 para o coeficiente L11 e zero para o coeficiente L12. Em seguida, atribui-se valor 1 para o coeficiente L12 e zero para o coeficiente L11 procedendo-se de forma análoga. Assim, tem-se para o fator $D$ a hipótese:

$$
H_{0}:\left\{\begin{array}{l}
\mu_{4} \cdot-\mu_{6}=0 \\
\mu_{5} \cdot-\mu_{6}=0
\end{array}\right.
$$

O teste associado a essa hipótese, verifica se as médias de produtividade dos híbridos duplos não diferem entre si. Os dois graus de liberdade para $D$ (Tabela 23) podem ser decompostos analogamente em:

Híbrido 4 vs. Híbrido 5, Híbrido 6 (1 g.l.)

Híbrido 5 vs. Híbrido 6 (1 g.l.)

Isso foi feito com o uso da função CONTRAST, obviamente levando aos mesmos resultados.

Para o fator $F$ :

$$
H_{0}:\left\{\begin{array}{l}
\mu_{7} \cdot-\mu_{9}=0 \\
\mu_{8} \cdot-\mu_{9}=0
\end{array}\right.
$$

O teste está associado à hipótese de que as médias de produtividade dos híbridos triplos não diferem entre si. Decompondo-se os graus de liberdade de $F$ em contrastes, tem-se:

Híbrido 7 vs. Híbrido 8, Híbrido 9 (1 g.l.)

Híbrido 8 vs. Híbrido 9 (1 g.l.) 
A análise de variância apresentada na Tabela 23, têm os 8 graus de liberdade no modelo com $\tau_{i}$ (Tabela 20) decompostos em 2 graus de liberdade para híbridos simples $(C), 2$ graus de liberdade para híbridos duplos $(D)$ e 2 graus de liberdade para híbridos triplos $(F)$. Neste caso, restam ainda 2 graus de liberdade, que correspondem às comparações entre HS, HD e HT. Os contrastes que representam estas comparações também podem ser testados por meio da função CONTRAST do PROC GLM, considerando conjuntamente os contrastes $\mathrm{Y}_{\mathrm{HS} \text { vs } \mathrm{HD} \text {, HT }}$ e $\mathrm{Y}_{\mathrm{HD}}$ vs HT.

Tabela 23. Análise de variância para o modelo $y=\mu+c_{i}+d_{i}+f_{i}+e_{i j}$.

\begin{tabular}{|c|c|c|c|c|c|}
\hline V.C.M. & G.L. & S.Q. & Q.M. & $\mathrm{F}$ & $\operatorname{Pr}>F$ \\
\hline Blocos & $(b-1)=3$ & $R(\beta \mid \mu, \tau)=0,0733$ & 0,0244 & 0,69 & 0,5654 \\
\hline$C$ & $\left(c^{\prime}-1\right)=2$ & $R(c \mid \mu, \beta, d, f)=1,2117$ & 0,6058 & 17,17 & 0,0001 \\
\hline$D$ & $\left(d^{\prime}-1\right)=2$ & $R(d \mid \mu, \beta, c, f)=1,5517$ & 0,7758 & 21,99 & 0,0001 \\
\hline$F$ & $\left(f^{\prime}-1\right)=2$ & $R(f \mid \mu, \beta, c, d)=3,5317$ & 1,7658 & 50,06 & 0,0001 \\
\hline Resíduo & $(n-t)=24$ & $\Delta=0,8467$ & 0,0353 & - & - \\
\hline Total & $(n-1)=35$ & $R(\mu, \beta, \tau)=7,2222$ & & - & - \\
\hline \multicolumn{6}{|c|}{$\begin{array}{l}\mu \text { : efeito da constante; } \beta \text { : efeito de blocos; } \tau \text { : efeito dos tratamentos; } c: \text { efeito dos híbridos do grupo } 1 \text {; } \\
d: \text { efeito dos híbridos do grupo } 2 ; f: \text { efeito dos híbridos do grupo } 3 ; b: \text { número de blocos; } c^{\prime}: \text { número } \\
\text { de níveis que assume a variável auxiliar } C \text {, exceto } c_{0} ; d^{\prime}: \text { número de níveis que assume a variável } \\
\text { auxiliar } D \text {, exceto } d_{0} ; f^{\prime}: \text { número de níveis que assume a variável auxiliar } F \text {, exceto } f_{0} . \Delta=R(\mu, \beta, \tau) \text { - } \\
R(\beta \mid \mu, \tau)-R(\tau \mid \mu, \beta) \text {. }\end{array}$} \\
\hline
\end{tabular}

Como o teste $\mathrm{F}$ para os fatores $C, D$ e $F$ foi significativo (Tabela 23), concluise que há diferença entre os efeitos de pelo menos duas variedades de cada um dos grupos de híbridos. Dessa forma, ainda há necessidade de construir contrastes para verificar quais híbridos diferem entre si dentro dos grupos.

A decomposição em contrastes ortogonais apresentada na Tabela 23, pode equivalentemente, ser testada pela função CONTRAST. Contudo, o uso de variáveis auxiliares torna a análise mais simples, pois com poucas linhas de programação faz-se a 
divisão dos tratamentos em grupos (codificação). Em seguida, escreve-se o modelo considerando as variáveis auxiliares e realiza-se a análise, não sendo necessário construir cada contraste, o que é muito trabalhoso em situações reais.

\subsection{Conjunto $\mathrm{C}$}

\subsubsection{Hipóteses testadas automaticamente pelo PROC GLM do SAS}

Com base no conjunto de dados $C$ (Tabela 7), foram discutidos os resultados fornecidos pelo PROC GLM do SAS, visando interpretar as hipóteses estatísticas e as somas de quadrados a elas associadas, considerando duas ordens dos fatores no modelo.

Apresentou-se para o conjunto de dados e modelo adotado, as funções estimáveis que possibilitam a construção das hipóteses. Nas Tabelas 24 e 25 são descritas as funções estimáveis, para os efeitos principais e interação, que são geradas e apresentadas automaticamente pelo PROC GLM.

Tabela 24. Funções estimáveis para o fator A, fornecidas pelo PROC GLM do SAS, para o conjunto de dados $C$ (Ordem A - B) .

\begin{tabular}{|c|c|c|c|c|c|c|}
\hline & & Tipo I & Tipo II & Tipo III & Tipo IV & Interação \\
\hline$\mu$ & 0 & 0 & 0 & 0 & 0 & 0 \\
\hline \multirow[t]{3}{*}{ A } & 1 & L2 & L2 & $\mathrm{L} 2$ & L2 & 0 \\
\hline & 2 & L3 & L3 & L3 & L3 & 0 \\
\hline & 3 & -L2-L3 & -L2-L3 & -L2-L3 & -L2-L3 & 0 \\
\hline \multirow[t]{2}{*}{ B } & 1 & $-0.5714 * \mathrm{~L} 2-0.5 * \mathrm{~L} 3$ & 0 & 0 & 0 & 0 \\
\hline & 2 & $0.5714 * \mathrm{~L} 2+0.5 * \mathrm{~L} 3$ & 0 & 0 & 0 & 0 \\
\hline \multirow[t]{5}{*}{$\mathrm{AB}$} & 11 & $0.4286 * \mathrm{~L} 2$ & $0.7333 * \mathrm{~L} 2+0.2667 * \mathrm{~L} 3$ & $0.75 * \mathrm{~L} 2+0.25 * \mathrm{~L} 3$ & L2 & L7 \\
\hline & 12 & $0.5714 * \mathrm{~L} 2$ & $0.2667 * \mathrm{~L} 2-0.2667 * \mathrm{~L} 3$ & $0.25 * \mathrm{~L} 2-0.25 * \mathrm{~L} 3$ & 0 & -L7 \\
\hline & 21 & $0.5 * \mathrm{~L} 3$ & $0.2667 * \mathrm{~L} 2+0.7333 * \mathrm{~L} 3$ & $0.25 * \mathrm{~L} 2+0.75 * \mathrm{~L} 3$ & L3 & $-\mathrm{L} 7$ \\
\hline & 22 & $0.5 * \mathrm{~L} 3$ & $0.2667 * \mathrm{~L} 2+0.2667 * \mathrm{~L} 3$ & $-0.25 * \mathrm{~L} 2+0.25 * \mathrm{~L} 3$ & 0 & L7 \\
\hline & 31 & -L2-L3 & -L2-L3 & -L2-L3 & -L2-L3 & 0 \\
\hline
\end{tabular}

Com o objetivo de utilizar as funções estimáveis do fator $A$ para reconhecer as hipóteses testadas, atribuiu-se valor 1 para o coeficiente L2 e zero para o coeficiente L3, 
construindo-se a primeira linha da hipótese. Em seguida, atribuiu-se valor zero para o coeficiente L2 e valor 1 para o coeficiente L3, construindo-se a segunda linha da hipótese. Procedendo-se da mesma forma para todas as funções estimáveis, construiu-se os quatro tipos de hipóteses para o fator $A$ que são apresentadas na Tabela 26.

Tabela 25. Funções estimáveis para o fator B, fornecidas pelo PROC GLM do SAS para o conjunto de dados $C$ (Ordem B - A).

\begin{tabular}{|c|c|c|c|c|c|c|}
\hline & & Tipo I & Tipo II & Tipo III & Tipo IV & Interação \\
\hline$\mu$ & 0 & 0 & 0 & 0 & 0 & 0 \\
\hline \multirow[t]{2}{*}{ B } & 1 & L2 & L2 & $\mathrm{L} 2$ & L2 & 0 \\
\hline & 2 & $-\mathrm{L} 2$ & $-\mathrm{L} 2$ & $-\mathrm{L} 2$ & $-\mathrm{L} 2$ & 0 \\
\hline \multirow[t]{3}{*}{ A } & 1 & $-0.1964 * \mathrm{~L} 2$ & 0 & 0 & 0 & 0 \\
\hline & 2 & $-0.0536 * \mathrm{~L} 2$ & 0 & 0 & 0 & 0 \\
\hline & 3 & $0.25 * \mathrm{~L} 2$ & 0 & 0 & 0 & 0 \\
\hline \multirow[t]{5}{*}{$\mathrm{AB}$} & 11 & $0.375 * \mathrm{~L} 2$ & $0.5333 * \mathrm{~L} 2$ & $0.5 * \mathrm{~L} 2$ & $0.5^{*} \mathrm{~L} 2$ & L7 \\
\hline & 12 & $-0.5714 * \mathrm{~L} 2$ & $-0.5333 * \mathrm{~L} 2$ & $-0.5 * \mathrm{~L} 2$ & $-0.5 * \mathrm{~L} 2$ & $-\mathrm{L} 7$ \\
\hline & 21 & $0.375^{*} \mathrm{~L} 2$ & $0.4667 * \mathrm{~L} 2$ & $0.5 * \mathrm{~L} 2$ & $0.5 * \mathrm{~L} 2$ & -L7 \\
\hline & 22 & $-0.4286 * \mathrm{~L} 2$ & $-0.4667^{*} \mathrm{~L} 2$ & $-0.5 * \mathrm{~L} 2$ & $-0.5 * \mathrm{~L} 2$ & L7 \\
\hline & $\begin{array}{ll}3 & 1\end{array}$ & $0.25 * \mathrm{~L} 2$ & 0 & 0 & 0 & 0 \\
\hline
\end{tabular}

Para o fator $B$, utilizaram-se as funções estimáveis para reconhecer as hipóteses testadas, atribuindo-se valor 1 para o coeficiente L2 (Tabela 27). De forma análoga para a hipótese sobre a interação, atribuindo-se valor 1 para o coeficiente L7.

Escrevendo-se os quatro tipos de hipóteses no modelo-S para os fatores $A$ e $B$ (Tabelas 26 e 27), percebe-se que todas as hipóteses envolvem os parâmetros referentes à interação. Assim, não se pode testar os efeitos principais isoladamente. As hipóteses do tipo I, por não sofrerem ajuste, apresentam coeficientes confusos, um contraste entre níveis do fator principal, seguido de um contraste entre níveis do outro fator e dos efeitos da interação e, dessa forma, aparentemente não têm interesse prático. Nas hipóteses do tipo II, apesar dos efeitos principais ajustados, há combinações lineares dos parâmetros relativos à interação e coeficientes pouco usuais, fatos que dificultariam a interpretação para usuários pouco experientes. 
Como há somente uma casela vazia e o fator $A$ possui três níveis, a hipótese do tipo III (Tabela 26) é de aparente interesse pois apresenta contrastes somente entre os efeitos do fator $A$ e coeficientes mais simples para a interação. Quando o número de caselas vazias e o número de níveis do fator aumentam, esta hipótese também se torna complexa.

As hipóteses do tipo IV são mais simples e fáceis de interpretar. Entretanto, há perda de informação, pois só é considerada uma parte dos dados, ou seja, a hipótese do tipo IV para o fator $A$ não considera qualquer observação da Cultivar M7 $\left(\gamma_{12}\right)$.

Essas situações são devidas ao desbalanceamento dos dados e, principalmente, à presença de caselas vazias.

Tabela 26. Hipóteses testadas sobre o fator A, no modelo superparametrizado.

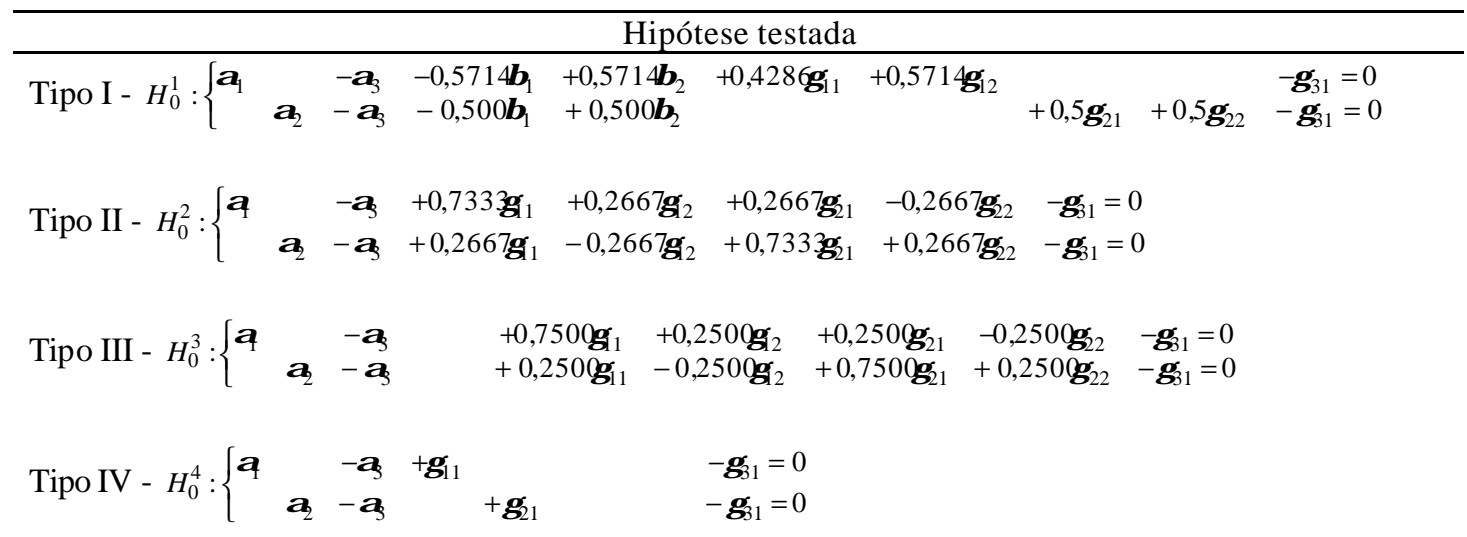

Interação - $H_{0}^{5}: \gamma_{11}-\gamma_{12}-\gamma_{21}+\gamma_{22}=0$

$\alpha_{1}$ : efeito do fator $A$, nível $1 ; \alpha_{2}$ : efeito do fator $A$, nível $2 ; \alpha_{3}$ : efeito do fator $A$, nível 3; $\beta_{1}$ : efeito do fator $B$, nível $1 ; \beta_{2}$ : efeito do fator $B$, nível $2 ; \gamma_{11}$ : efeito da interação entre o nível 1 do fator $A$ e o nível 1 do fator $B ; \gamma_{12}$ : efeito da interação entre o nível 1 do fator $A$ e o nível 2 do fator $B ; \gamma_{21}$ : efeito da interação entre o nível 2 do fator $A$ e o nível 1 do fator $B ; \gamma_{22}$ : efeito da interação entre o nível 2 do fator $A$ e o nível 2 do fator $B ; \gamma_{31}$ : efeito da interação entre o nível 3 do fator $A$ e o nível 1 do fator $B$. 
Tabela 27. Hipóteses testadas sobre o fator B no modelo superparametrizado para o conjunto de dados $C$.

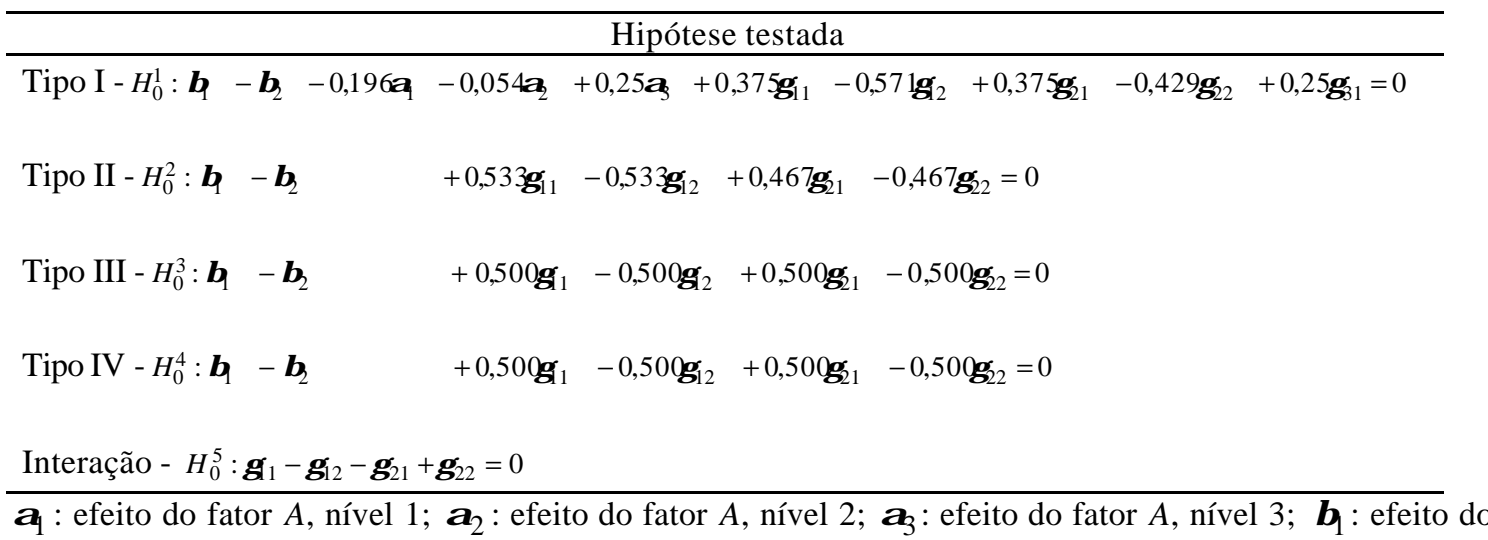
fator $B$, nível 1; $\beta_{2}$ : efeito do fator $B$, nível 2; $\gamma_{11}$ : efeito da interação entre o nível 1 do fator $A$ e o nível 1 do fator $B ; \gamma_{12}$ : efeito da interação entre o nível 1 do fator $A$ e o nível 2 do fator $B ; \gamma_{21}$ : efeito da interação entre o nível 2 do fator $A$ e o nível 1 do fator $B ; \gamma_{22}$ : efeito da interação entre o nível 2 do fator $A$ e o nível 2 do fator $B ; \gamma_{31}$ : efeito da interação entre o nível 3 do fator $A$ e o nível 1 do fator $B$.

Para esse experimento percebe-se que para o fator $B$, a hipótese do tipo III é equivalente à hipótese do tipo IV (Tabela 27), apesar da casela vazia. Isso ocorre pois o fator $B$ tem somente dois níveis e, conseqüentemente, a matriz hipótese possui apenas uma linha (1 grau de liberdade).

Para o conjunto de dados da Tabela 7, se todas as caselas fossem ocupadas, a hipótese sobre a interação teria 2 graus de liberdade (duas linhas) pois, conforme abordado em 2.6.2, esse número é dado por $(a-1)(b-1)=(3-1)(2-1)=2$. Entretanto, como há uma casela vazia, perde-se um grau de liberdade para interação, ou seja, há $(a-1)(b-1)-v=2-1=1$ graus de liberdade e a hipótese possui somente uma linha (Tabelas 26 e 27).

A hipótese sobre a interação (Tabelas 26 e 27) é livre de parâmetros sem interesse. Entretanto, essa hipótese testa que somente algumas interações são nulas (a interação $\gamma_{31}$ não faz parte da hipótese), o que não permite que seja utilizada como um critério de escolha entre um modelo com ou sem interação. A escolha pode ser feita pelo 
pesquisador baseado em sua experiência sobre os fatores estudados, o que nem sempre é possível e recomendado.

Com a apresentação dos resultados das análises de variância, considerando diferentes ordens do modelo (Tabelas 28 e 29) confirma-se que as somas de quadrados do tipo I, fornecidas pelo PROC GLM do SAS, dependem da ordem de entrada dos parâmetros no modelo, dado que são obtidas seqüencialmente. Assim, tem-se um valor para soma de quadrados do tipo I para os fatores $A$ e $B$ na ordem $A-B$ e outro na ordem $B$ - $A$. Para as demais somas de quadrados, a ordem dos fatores no modelo não altera o resultado.

Tabela 28. Somas de quadrados fornecidas pelo PROC GLM, ordem A - B.

\begin{tabular}{|c|c|c|c|c|c|}
\hline V.C.M. & G.L. & $S Q$ Tipo I & $Q M$ & $F$ & $\operatorname{Pr}>F$ \\
\hline$\overline{\mathrm{A}}$ & 2 & 6.53945190 & 3.26972595 & 59.86 & $<.0001$ \\
\hline B & 1 & 0.38322134 & 0.38322134 & 7.02 & 0.0244 \\
\hline$A * B$ & 1 & 0.32376642 & 0.32376642 & 5.93 & 0.0352 \\
\hline V.C.M. & G.L. & SQ Tipo II & $Q M$ & $F$ & $\operatorname{Pr}>F$ \\
\hline A & 2 & 5.71730134 & 2.85865067 & 52.33 & $<.0001$ \\
\hline B & 1 & 0.38322134 & 0.38322134 & 7.02 & 0.0244 \\
\hline$A * B$ & 1 & 0.32376642 & 0.32376642 & 5.93 & 0.0352 \\
\hline V.C.M. & G.L. & SQ Tipo III & $Q M$ & $F$ & $\operatorname{Pr}>F$ \\
\hline A & 2 & 5.52082202 & 2.76041101 & 50.53 & $<.0001$ \\
\hline B & 1 & 0.33609602 & 0.33609602 & 6.15 & 0.0325 \\
\hline$A * B$ & 1 & 0.32376642 & 0.32376642 & 5.93 & 0.0352 \\
\hline V.C.M. & G.L. & SQ Tipo IV & $Q M$ & $F$ & $\operatorname{Pr}>F$ \\
\hline A & 2 & 1.75339533 & 0.87669767 & 16.05 & 0.0008 \\
\hline B & 1 & 0.33609602 & 0.33609602 & 6.15 & 0.0325 \\
\hline$A * B$ & 1 & 0.32376642 & 0.32376642 & 5.93 & 0.0352 \\
\hline
\end{tabular}


Tabela 29. Somas de quadrados fornecidas pelo PROC GLM, ordem B - A.

\begin{tabular}{|c|c|c|c|c|c|}
\hline V.C.M. & G.L. & SQ Tipo I & $Q M$ & $F$ & $\operatorname{Pr}>F$ \\
\hline $\bar{B}$ & 1 & 1.20537190 & 1.20537190 & 22.07 & 0.0008 \\
\hline A & 2 & 5.71730134 & 2.85865067 & 52.33 & $<.0001$ \\
\hline$A * B$ & 1 & 0.32376642 & 0.32376642 & 5.93 & 0.0352 \\
\hline$V \cdot C \cdot M$. & $G . L$. & SQ Tipo II & $Q M$ & $F$ & $\operatorname{Pr}>F$ \\
\hline B & 1 & 0.38322134 & 0.38322134 & 7.02 & 0.0244 \\
\hline A & 2 & 5.71730134 & 2.85865067 & 52.33 & $<.0001$ \\
\hline$A * B$ & 1 & 0.32376642 & 0.32376642 & 5.93 & 0.0352 \\
\hline$V \cdot C \cdot M$. & $G . L$. & $S Q$ Tipo III & $Q M$ & $F$ & $\operatorname{Pr}>F$ \\
\hline B & 1 & 0.33609602 & 0.33609602 & 6.15 & 0.0325 \\
\hline A & 2 & 5.52082202 & 2.76041101 & 50.53 & $<.0001$ \\
\hline$A \star B$ & 1 & 0.32376642 & 0.32376642 & 5.93 & 0.0352 \\
\hline$V \cdot C \cdot M$. & $G . L$. & $S Q$ Tipo IV & $Q M$ & $F$ & $\operatorname{Pr}>F$ \\
\hline B & 1 & 0.33609602 & 0.33609602 & 6.15 & 0.0325 \\
\hline A & 2 & 1.75339533 & 0.87669767 & 16.05 & 0.0008 \\
\hline$A * B$ & 1 & 0.32376642 & 0.32376642 & 5.93 & 0.0352 \\
\hline
\end{tabular}

No PROC GLM do SAS, as somas de quadrados dos tipos I, II e III são únicas, o que não ocorre para a do tipo IV. Dessa forma, com uma reordenação dos dados é possível obter outras funções estimáveis do tipo IV, o que levará a outras somas de quadrados, dificultando ainda mais as interpretações.

Frente à diversidade de somas de quadrados apresentadas nas Tabelas 28 e 29, o pesquisador ao utilizar o PROC GLM, pode optar pela análise de variância mais apropriada para a situação experimental. Além disso, entende-se que é indispensável que os manuais de sistemas estatísticos sejam esclarecedores, minimizando erros de interpretação de hipóteses em análises de dados.

\subsubsection{Testes de hipóteses usando a função CONTRAST}

Diante da dificuldade na interpretação das hipóteses apresentadas pelo PROC GLM, para dados desbalanceados e com caselas vazias, o pesquisador tem como opção construir hipóteses de interesse e testá-las diretamente utilizando a função CONTRAST do SAS. Aqui, utilizaram-se como exemplo, as funções estimáveis do tipo III e algumas 
hipóteses de suposto interesse para os fatores A e B, como exposto em Material e Métodos.

a) Hipótese apenas sobre fator A:

Em situações práticas, é usual o pesquisador desejar testar uma hipótese sobre efeito principal de $A$ isoladamente. Essa hipótese tem como função paramétrica $B^{\prime} \boldsymbol{\theta}=\phi$, em que

$$
B^{\prime}=\left[\begin{array}{lllllllllll}
0 & 1 & 0 & -1 & 0 & 0 & 0 & 0 & 0 & 0 & 0 \\
0 & 0 & 1 & -1 & 0 & 0 & 0 & 0 & 0 & 0 & 0
\end{array}\right]
$$

é a matriz com os coeficientes da função paramétrica, adequada para testar essa hipótese.

No presente caso, uma vez que há interação, a função CONTRAST não calcula qualquer soma de quadrados e informa o usuário sobre a não estimabilidade da função paramétrica, da seguinte forma:

NOTE: CONTRAST $Y($ Apenas $f$ at or A) is not esti mable.

Como a função paramétrica que descreve o contraste não é estimável, a hipótese correspondente não é testável. Assim sendo, o pesquisador deverá construir outras hipóteses, se optar por não usar diretamente as fornecidas pelo SAS.

b) Hipótese do Tipo III para o fator A:

Essa hipótese tem como função paramétrica $B^{\prime} \boldsymbol{\theta}=\boldsymbol{\phi}$, em que

$$
B^{\prime}=\left[\begin{array}{ccccccccccc}
0 & 1 & 0 & -1 & 0 & 0 & 0,75 & 0,25 & 0,25 & -0,25 & -1 \\
0 & 0 & 1 & -1 & 0 & 0 & 0,25 & -0,25 & 0,75 & 0,25 & -1
\end{array}\right]
$$

é a matriz com os coeficientes da função paramétrica.

Utilizando-se $B^{\prime}$ na função CONTRAST do PROC GLM, conforme abordado em 3.2.4.3, tem-se a seguinte hipótese com teste F (programa SAS no APÊNDICE): 


\begin{tabular}{lrrrrr} 
Cont rast & DF & Cont rast SS & Mean Square & $F$ Val ue & Pr $>F$ \\
\hline Y(Fat or A & 2 & 5.52082202 & 2.76041101 & 50.53 & 0.0001
\end{tabular}

Essa análise de variância é fornecida pelo programa, e percebe-se que são os mesmos resultados obtidos de forma convencional pelo PROC GLM (Tabela 28), quando a soma de quadrados para o fator $A$ é solicitada. Nota-se que não é uma tarefa simples encontrar hipóteses diferentes das oferecidas diretamente pelo SAS.

c) Hipótese apenas sobre fator B:

A hipótese sobre o efeito principal de $B$, no modelo-S, tem como função paramétrica $B^{\prime} \boldsymbol{\theta}=\boldsymbol{\phi}$, em que

$$
B^{\prime}=\left[\begin{array}{lllllllllll}
0 & 1 & -1 & 0 & 0 & 0 & 0 & 0 & 0 & 0 & 0
\end{array}\right]
$$

é a matriz dos coeficientes da função paramétrica.

Como ocorreu para a hipótese sobre apenas fator A, a função CONTRAST não executa a análise de variância e informa o usuário sobre a não estimabilidade da função, e em conseqüência que a hipótese não é testável:

NOTE: CONTRAST Y(Apenas Fat or B) is not est i mabl e.

d) Hipótese do Tipo III para o fator B:

A hipótese $H_{0}^{3-B}$, tem como função paramétrica $B^{\prime} \theta=\phi$, em que

$$
B^{\prime}=\left[\begin{array}{lllllllllll}
0 & 1 & -1 & 0 & 0 & 0 & 0,5 & -0,5 & 0,5 & -0,5 & 0
\end{array}\right]
$$

é a matriz com os coeficientes da função paramétrica.

A função CONTRAST do PROC GLM realiza a análise de variância dessa hipótese (programa no APÊNDICE) obtendo-se como resultado: 


\begin{tabular}{lrrrrr} 
Contrast & DF & Contrast SS & Mean Square & F Value & Pr $>$ F \\
\hline Y(Fat or B) & 1 & 0.33609602 & 0.33609602 & 6.15 & 0.032
\end{tabular}

Novamente, esse resultado é o mesmo obtido pelo PROC GLM (Tabela 29) quando a soma de quadrados do tipo III é solicitada. Nota-se que é difícil construir hipóteses diferentes daquelas já fornecidas pelo SAS.

\subsubsection{Testes de hipóteses pela técnica da restrição paramétrica estimável}

A técnica da restrição paramétrica estimável é uma opção para a realização de testes de hipóteses de interesse. Novamente, supôs-se que o pesquisador tem interesse em verificar se hipóteses de seu interesse são testáveis, sem se preocupar em verificar se o que o SAS fornece é de interesse imediato. Assim, foram supostas algumas situações práticas, discutidas a seguir.

a) Hipóteses do tipo III para o Fator $A$

A hipótese $H_{0}^{3-A}$ escrita no modelo-S (Tabela 26), tem como função paramétrica $B^{\prime} \boldsymbol{\theta}=\phi$, em que

$$
B^{\prime}=\left[\begin{array}{ccccccccccc}
0 & 1 & 0 & -1 & 0 & 0 & 0,75 & 0,25 & 0,25 & -0,25 & -1 \\
0 & 0 & 1 & -1 & 0 & 0 & 0,25 & -0,25 & 0,75 & 0,25 & -1
\end{array}\right]
$$

é a matriz com os coeficientes da função paramétrica.

Imagina-se que, uma vez que não foi possível testar a hipótese apresentada em 4.3.2 (a), o pesquisador construiu agora outra hipótese.

Para o conjunto de dados da Tabela 7, e o modelo superparametrizado caracterizado em (31), obteve-se o modelo linear na forma matricial $(y=X \boldsymbol{\theta}+e)$ : 


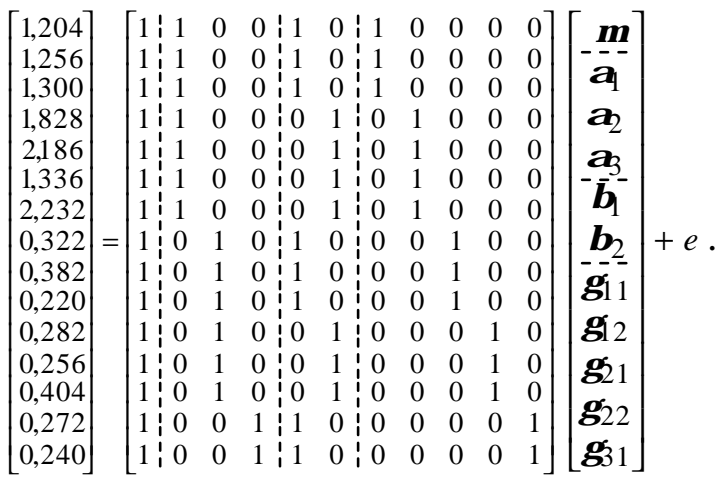

Na matriz $X$, a primeira coluna denota o efeito da constante, as colunas 2,3 e 4 denotam os efeitos dos níveis do fator A, as colunas 5 e 6 denotam os efeitos dos níveis de B. As demais colunas denotam os efeitos da interação.

Considerando-se a forma geral

$$
\left[\begin{array}{c:c}
X^{\prime} X & B \\
\hdashline B^{\prime} & \phi
\end{array}\right]\left[\begin{array}{l}
\theta \\
-l
\end{array}\right]=\left[\begin{array}{c}
X^{\prime} y \\
\hdashline \phi
\end{array}\right]
$$

construiu-se o sistema de equações normais com a restrição paramétrica, para o presente caso:

$$
\left[\begin{array}{ccccccccccc:cr}
15 & 7 & 6 & 2 & 8 & 7 & 3 & 4 & 3 & 3 & 2 & 0 & 0 \\
7 & 7 & 0 & 0 & 3 & 4 & 3 & 4 & 0 & 0 & 0 & 1 & 0 \\
6 & 0 & 6 & 0 & 3 & 3 & 0 & 0 & 3 & 3 & 0 & 0 & 1 \\
2 & 0 & 0 & 2 & 2 & 0 & 0 & 0 & 0 & 0 & 2 & -1 & -1 \\
8 & 3 & 3 & 2 & 8 & 0 & 3 & 0 & 3 & 0 & 2 & 0 & 0 \\
7 & 4 & 3 & 0 & 0 & 7 & 0 & 4 & 0 & 3 & 0 & 0 & 0 \\
3 & 3 & 0 & 0 & 3 & 0 & 3 & 0 & 0 & 0 & 0 & 0,75 & 0,25 \\
4 & 4 & 0 & 0 & 0 & 4 & 0 & 4 & 0 & 0 & 0 & 0,25 & -0,25 \\
3 & 0 & 3 & 0 & 3 & 0 & 0 & 0 & 3 & 0 & 0 & 0,25 & 0,75 \\
3 & 0 & 3 & 0 & 0 & 3 & 0 & 0 & 0 & 3 & 0 & -0,25 & 0,25 \\
2 & 0 & 0 & 2 & 2 & 0 & 0 & 0 & 0 & 0 & 2 & -1 & -1 \\
\hdashline 0 & 1 & 0 & -1 & 0 & 0 & 0,75 & 0,25 & 0,25 & -0,25 & -1 & 0 & 0 \\
0 & 0 & 1 & -1 & 0 & 0 & 0,25 & -0,25 & 0,75 & 0,25 & -1 & 0 & 0
\end{array}\right]\left[\tilde{\boldsymbol{\theta}} \cdot\left[\begin{array}{c}
13,716 \\
11,338 \\
1,966 \\
0,512 \\
5,196 \\
8,520 \\
3,760 \\
7,578 \\
0,924 \\
0,942 \\
0,512 \\
\hdashline 0 \\
0
\end{array}\right]\right.
$$

Vale lembrar que $B^{\prime}$ é agora uma restrição linearmente dependente, conforme detalhado no item 2.5.1 da revisão.

$$
\text { Fazendo-se }\left[\begin{array}{c}
\tilde{\boldsymbol{\theta}} \\
\hdashline l
\end{array}\right]=\left[\begin{array}{c:c}
X^{\prime} X & B \\
\hdashline B^{\prime} & \phi
\end{array}\right]^{G}\left[\begin{array}{c}
X^{\prime} y \\
\hdashline \phi
\end{array}\right] \text { obteve-se: }
$$




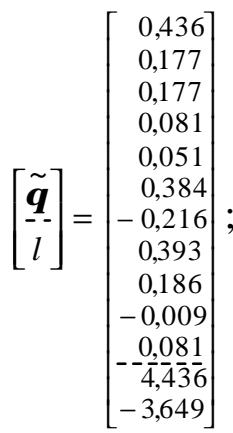

$$
\tilde{\boldsymbol{\Theta}}=\left[\begin{array}{r}
0,436 \\
0,177 \\
0,177 \\
0,081 \\
0,051 \\
0,384 \\
-0,216 \\
0,393 \\
0,186 \\
-0,009 \\
0,081
\end{array}\right]
$$

em que $\tilde{\boldsymbol{\theta}}$ é a solução do sistema de equações normais com a restrição paramétrica $B^{\prime} \boldsymbol{\theta}=\boldsymbol{\phi}$. Para tanto, utilizou-se a inversa generalizada de Moore-Penrose.

Calculou-se $\tilde{y}=X \tilde{\boldsymbol{\theta}}$, que é a aproximação de mínimos quadrados para o vetor $y$ de observações:

$$
\tilde{y}=\left[\begin{array}{lllll:ll:lllll}
1 & 1 & 0 & 0 & 1 & 0 & 1 & 0 & 0 & 0 & 0 \\
1 & 1 & 0 & 0 & 1 & 0 & 1 & 0 & 0 & 0 & 0 \\
1 & 1 & 1 & 0 & 0 & 1 & 0 & 1 & 0 & 0 & 0 & 0 \\
1 & 1 & 0 & 0 & 0 & 1 & 0 & 1 & 0 & 0 & 0 \\
1 & 1 & 0 & 0 & 0 & 1 & 0 & 1 & 0 & 0 & 0 \\
1 & 1 & 0 & 0 & 0 & 1 & 0 & 1 & 0 & 0 & 0 \\
1 & 1 & 1 & 0 & 0 & 0 & 1 & 0 & 1 & 0 & 0 & 0 \\
1 & 0 & 1 & 0 & 1 & 0 & 0 & 0 & 1 & 0 & 0 \\
1 & 0 & 1 & 0 & 1 & 0 & 0 & 0 & 1 & 0 & 0 \\
1 & 0 & 1 & 0 & 1 & 0 & 0 & 0 & 1 & 0 & 0 \\
1 & 0 & 1 & 0 & 0 & 1 & 0 & 0 & 0 & 1 & 0 \\
1 & 0 & 1 & 0 & 0 & 1 & 0 & 0 & 0 & 1 & 0 \\
1 & 0 & 1 & 0 & 0 & 1 & 0 & 0 & 0 & 1 & 0 \\
1 & 0 & 0 & 1 & 1 & 0 & 0 & 0 & 0 & 0 & 1 \\
1 & 0 & 0 & 1 & 1 & 0 & 0 & 0 & 0 & 0 & 1
\end{array}\right]\left[\begin{array}{r}
0,436 \\
0,177 \\
0,177 \\
0,081 \\
0,051 \\
0,384 \\
-0,216 \\
0,393 \\
0,186 \\
-0,009 \\
0,081
\end{array}\right] ; \quad \tilde{y}=\left[\begin{array}{l} 
\\
0,448 \\
1,390 \\
1,390 \\
1,390 \\
1,390 \\
0,851 \\
0,851 \\
0,851 \\
0,988 \\
0,988 \\
0,988 \\
0,649 \\
0,649
\end{array}\right]
$$

A seguir, calculou-se $\tilde{e}$, que é o erro de ajustamento devido à solução $\tilde{\boldsymbol{\theta}}$ : 


$\tilde{e}=y-\tilde{y}=\left[\begin{array}{r}1,204 \\ 1,256 \\ 1,300 \\ 1,828 \\ 2,186 \\ 1,332 \\ 2,232 \\ 0,322 \\ 0,382 \\ 0,22 \\ 0,222 \\ 0,256 \\ 0,404 \\ 0,272 \\ 0,240\end{array}\right]-\left[\begin{array}{l}0,448 \\ 0,448 \\ 0,448 \\ 1,390 \\ 1,390 \\ 1,390 \\ 1,390 \\ 0,851 \\ 0,851 \\ 0,851 \\ 0,988 \\ 0,988 \\ 0,988 \\ 0,649 \\ 0,649\end{array}\right]=\left[\begin{array}{r}0,756 \\ 0,808 \\ 0,852 \\ 0,438 \\ 0,796 \\ -0,054 \\ 0,842 \\ -0,529 \\ -0,469 \\ -0,631 \\ -0,706 \\ -0,732 \\ -0,558 \\ -0,377 \\ -0,409\end{array}\right]$

Fazendo-se $\tilde{e}^{\prime} \tilde{e}=\|\tilde{e}\|^{2}=S Q_{\tilde{\operatorname{Re}} s}$, obteve-se $S Q_{\tilde{\operatorname{Re}} s}=6,067$ que é a soma de quadrados do resíduo para o modelo com a restrição paramétrica $B^{\prime} \boldsymbol{\theta}=\boldsymbol{\phi}$.

Agora, para o cálculo da soma de quadrados usual do resíduo $\left(S Q_{\operatorname{Re} s}\right)$, calculouse $\hat{y}=X \boldsymbol{\theta}^{0}$, ou seja, a aproximação de mínimos quadrados para o vetor $y$ de observações (sem restrição paramétrica):

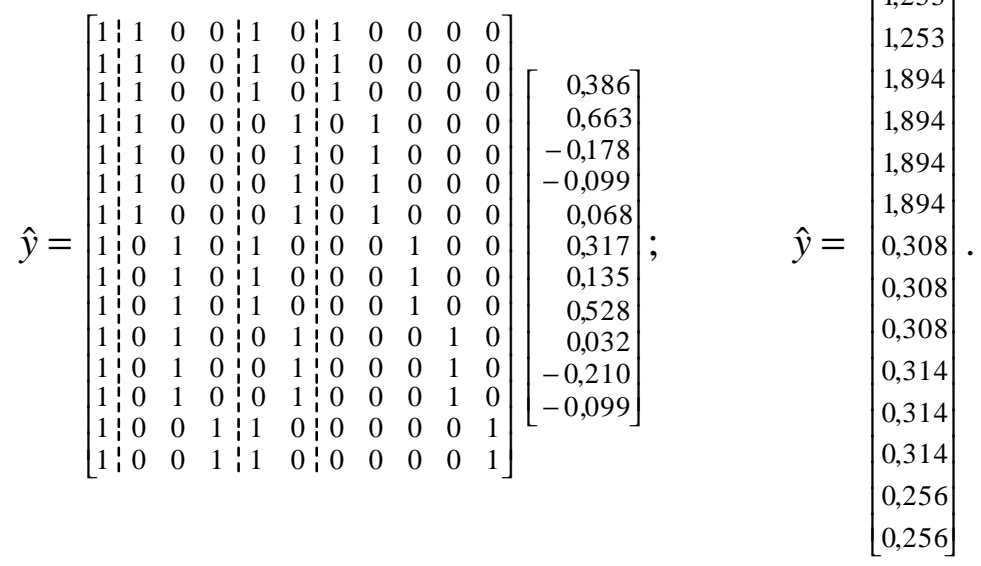

Utilizando-se $\hat{y}$, calculou-se o erro de ajustamento $(\hat{e})$ : 


$$
\hat{e}=y-\hat{y}=\left[\begin{array}{l}
1,204 \\
1,256 \\
1,300 \\
1,828 \\
2,186 \\
1,332 \\
2,232 \\
0,322 \\
0,382 \\
0,22 \\
0,282 \\
0,256 \\
0,404 \\
0,272 \\
0,240
\end{array}\right]-\left[\begin{array}{r}
1,253 \\
1,253 \\
1,253 \\
1,894 \\
1,894 \\
1,1,894 \\
1,394 \\
0,308 \\
0,308 \\
0,308 \\
0,314 \\
0,314 \\
0,314 \\
0,256 \\
0,256
\end{array}\right]=\left[\begin{array}{r}
-0,049 \\
0,003 \\
0,047 \\
-0,066 \\
0,291 \\
-0,562 \\
0,337 \\
0,014 \\
0,074 \\
-0,088 \\
-0,032 \\
-0,058 \\
0,090 \\
0,016 \\
-0,016
\end{array}\right] .
$$

Efetuando-se $\hat{e}^{\prime} \hat{e}=\|\hat{e}\|^{2}=S Q_{\mathrm{Re} s}$, obteve-se $S Q_{\mathrm{Re} s}=0,546$, que é o valor da soma de quadrados usual do resíduo.

Por diferença, o acréscimo $(\boldsymbol{\delta})$ na soma de quadrados usual do resíduo foi:

$$
\delta=S Q_{\tilde{\operatorname{Re}} s}-S Q_{\operatorname{Re} s}=6,067-0,546=5,520 .
$$

Esse valor $\delta=5,520$ é o acréscimo verificado na $S Q_{\operatorname{Re} s}$ motivado pela imposição da restrição paramétrica. Nesse caso, como $\delta>0$, conclui-se que a restrição paramétrica é linearmente dependente e, em consequiência, é estimável. Assim, a hipótese é testável, e $\delta$ corresponde à soma de quadrados associada à hipótese $H_{0}^{3-A}$, ou seja, $\delta=S Q_{H_{0}^{3-A}}=5,520$.

Fazendo-se:

$$
F_{\text {obs }}=\frac{\frac{5,520}{2}}{0,0546}=50,54,
$$

obteve-se o valor da estatística $F$ para a hipótese $H_{0}^{3-A}$.

Os resultados obtidos por meio da técnica da restrição paramétrica estão de acordo com o que foi encontrado utilizando-se o PROC GLM do SAS de forma convencional (Tabela 28), e com o resultado obtido por meio da função CONTRAST, sendo a pequena diferença encontrada devida a arredondamentos. Como esperado, isso mostra que o pesquisador pode optar por construir diretamente uma hipótese de interesse, obtendo em seguida sua soma de quadrados pela técnica da restrição 
paramétrica estimável ou pela função CONTRAST. A interpretação desse resultado é análoga ao que já foi discutido.

Com o objetivo de facilitar a realização dos cálculos elaborou-se um programa para ser implementado no SAS (ver APÊNDICE), facilitando ao usuário sua aplicação.

b) Hipótese apenas sobre efeito do fator $\mathrm{A}$

Imaginando que o pesquisador tem interesse numa hipótese simples sobre o fator A, como discutido em 4.3.2, os resultados obtidos com a restrição são discutidos a seguir.

A hipótese $H_{0}^{*-A}$ sobre o efeito principal de $A$, escrita no modelo-S sem considerar a interação, tem como função paramétrica $B^{\prime} \boldsymbol{\theta}=\boldsymbol{\phi}$, em que

$$
B^{\prime}=\left[\begin{array}{lllllllllll}
0 & 1 & 0 & -1 & 0 & 0 & 0 & 0 & 0 & 0 & 0 \\
0 & 0 & 1 & -1 & 0 & 0 & 0 & 0 & 0 & 0 & 0
\end{array}\right]
$$

é a matriz com os coeficientes da função paramétrica.

O modelo linear na forma matricial corresponde ao apresentado em (36). Assim, o sistema de equações normais com a restrição paramétrica em sua forma geral, é

$$
\left[\begin{array}{c:c}
X^{\prime} X & B \\
\hdashline B^{\prime} & \phi
\end{array}\right]\left[\begin{array}{l}
\boldsymbol{\theta} \\
-l
\end{array}\right]=\left[\begin{array}{c}
X^{\prime} y \\
\phi
\end{array}\right] .
$$

No caso, os valores são:

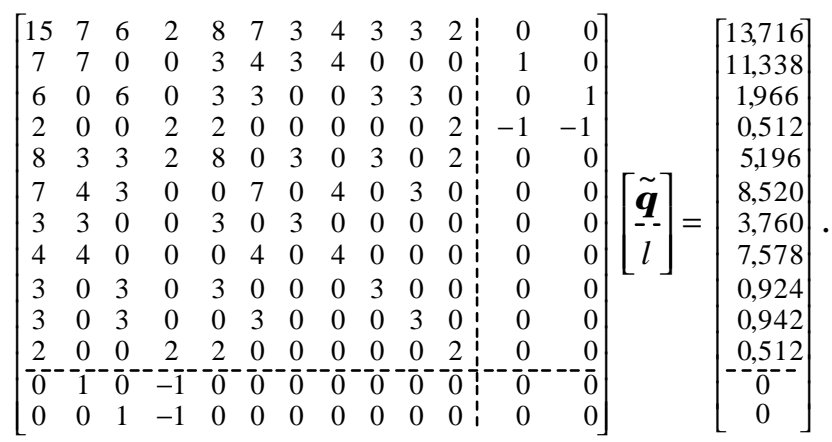


Fazendo-se

$$
\left[\begin{array}{c}
\tilde{\boldsymbol{\Theta}} \\
\hdashline l
\end{array}\right]=\left[\begin{array}{c:c}
X^{\prime} X & B \\
\hdashline B^{\prime} & \phi
\end{array}\right]^{G}\left[\begin{array}{c}
X^{\prime} y \\
\hdashline \phi
\end{array}\right]
$$

obtém-se $\tilde{\boldsymbol{\theta}}$, que é a solução do sistema de equações normais com a restrição paramétrica (utilizou-se a inversa generalizada de Moore-Penrose):

$$
\tilde{\boldsymbol{\Theta}}=\left[\begin{array}{c}
0,412 \\
0,137 \\
0,137 \\
0,137 \\
0,042 \\
0,370 \\
0,662 \\
0,976 \\
-0,284 \\
-0,606 \\
-0,336
\end{array}\right]
$$

Calculando-se a aproximação para o vetor $y$ de observações ( $\tilde{y}$ ), como em (39), obteve-se:

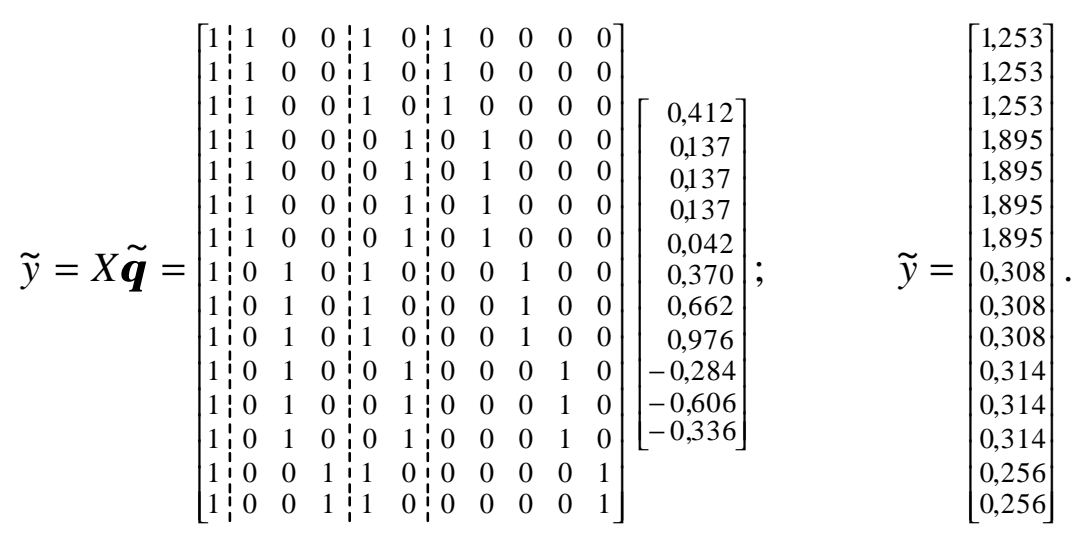

Fazendo-se $\tilde{e}=y-\tilde{y}$ e $\tilde{e}^{\prime} \tilde{e}=S Q_{\tilde{\operatorname{Re}} s}$ tem-se que $S Q_{\tilde{\operatorname{Re}} s}=0,546$ é a soma de quadrados do resíduo para o modelo com a restrição paramétrica $B^{\prime} \boldsymbol{\theta}=\boldsymbol{\phi}$.

Agora, para o cálculo da soma de quadrados usual do resíduo $\left(S Q_{\operatorname{Re} s}\right)$, obtém-se $\hat{y}=X \boldsymbol{\theta}^{0}$, ou seja, a aproximação de mínimos quadrados para o vetor $y$ de observações (sem restrição paramétrica): 


$$
\hat{y}=\left[\begin{array}{lllllllllll}
1 & 1 & 0 & 0 & 1 & 0 & 1 & 0 & 0 & 0 & 0 \\
1 & 1 & 0 & 0 & 1 & 0 & 1 & 0 & 0 & 0 & 0 \\
1 & 1 & 0 & 0 & 1 & 0 & 1 & 0 & 0 & 0 & 0 \\
1 & 1 & 0 & 0 & 0 & 1 & 0 & 1 & 0 & 0 & 0 \\
1 & 1 & 0 & 0 & 0 & 1 & 0 & 1 & 0 & 0 & 0 \\
1 & 1 & 0 & 0 & 0 & 1 & 0 & 1 & 0 & 0 & 0 \\
1 & 1 & 0 & 0 & 0 & 1 & 0 & 1 & 0 & 0 & 0 \\
1 & 0 & 1 & 0 & 1 & 0 & 0 & 0 & 1 & 0 & 0 \\
1 & 0 & 1 & 0 & 1 & 0 & 0 & 0 & 1 & 0 & 0 \\
1 & 0 & 1 & 0 & 1 & 0 & 0 & 0 & 1 & 0 & 0 \\
1 & 0 & 1 & 0 & 0 & 1 & 0 & 0 & 0 & 1 & 0 \\
1 & 0 & 1 & 0 & 0 & 1 & 0 & 0 & 0 & 1 & 0 \\
1 & 0 & 1 & 0 & 0 & 1 & 0 & 0 & 0 & 1 & 0 \\
1 & 0 & 0 & 1 & 1 & 0 & 0 & 0 & 0 & 0 & 1 \\
1 & 0 & 0 & 1 & 1 & 0 & 0 & 0 & 0 & 0 & 1
\end{array}\right]\left[\begin{array}{r}
0,386 \\
0,663 \\
-0,178 \\
-0,099 \\
0,068 \\
0,317 \\
0,135 \\
0,528 \\
0,032 \\
-0,210 \\
-0,099
\end{array}\right] ; \quad \hat{y}=\left[\begin{array}{r}
1,253 \\
1,253 \\
1,894 \\
1,894 \\
1,894 \\
1,894 \\
0,308 \\
0,308 \\
0,308 \\
0,314 \\
0,314 \\
0,314 \\
0,256 \\
0,256
\end{array}\right] .
$$

Utilizando-se $\hat{y}$, calculou-se o erro de ajustamento $(\hat{e})$ :

$$
\hat{e}=y-\hat{y}=\left[\begin{array}{l}
1,204 \\
1,256 \\
1,300 \\
1,828 \\
2,186 \\
1,332 \\
2,232 \\
0,322 \\
0,382 \\
0,22 \\
0,282 \\
0,256 \\
0,404 \\
0,272 \\
0,240
\end{array}\right]-\left[\begin{array}{l}
1,253 \\
1,253 \\
1,253 \\
1,894 \\
1,894 \\
1,894 \\
1,894 \\
0,308 \\
0,308 \\
0,308 \\
0,314 \\
0,314 \\
0,314 \\
0,256 \\
0,256
\end{array}\right]=\left[\begin{array}{r}
-0,049 \\
0,003 \\
0,047 \\
-0,066 \\
0,291 \\
-0,562 \\
0,337 \\
0,014 \\
0,074 \\
-0,088 \\
-0,032 \\
-0,058 \\
0,090 \\
0,016 \\
-0,016
\end{array}\right] .
$$

Assim, $\hat{e}^{\prime} \hat{e}=\|\hat{e}\|^{2}=S Q_{\operatorname{Re} s}=0,546$ é a soma de quadrados usual do resíduo.

Verificando-se $\delta$ por diferença:

$$
\delta=S Q_{\tilde{\operatorname{Re} s}}-S Q_{\operatorname{Re} s}=0,546-0,546=0 .
$$

Logo, $\delta=0$ é o acréscimo verificado na $S Q_{\operatorname{Re} s}$ motivado pela imposição da restrição paramétrica. Como a restrição paramétrica é linearmente independente, ela não é estimável. Em conseqüência, o pesquisador não conseguirá testar a hipótese $H_{0}^{*-A}$. Com isso, o pesquisador necessitará entender a estrutura dos seus dados, percebendo que a perda de caselas traz problemas, como por exemplo, a impossibilidade de testar os 
efeitos dos fatores principais isoladamente, caso a interação seja significativa. É importante destacar que esses resultados foram os mesmos que os obtidos com a função CONTRAST.

c) Hipótese apenas sobre efeito do fator B

A hipótese $H_{0}^{*-B}$ apenas sobre o efeito de $B$ no modelo-S, tem como função paramétrica $B^{\prime} \boldsymbol{\theta}=\boldsymbol{\phi}$, em que

$$
B^{\prime}=\left[\begin{array}{lllllllllll}
0 & 1 & -1 & 0 & 0 & 0 & 0 & 0 & 0 & 0 & 0
\end{array}\right]
$$

é a matriz dos coeficientes da função paramétrica.

Para o conjunto de dados e modelo superparametrizado caracterizado em (23), tem-se o modelo linear na forma matricial $(y=X \boldsymbol{\theta}+e)$ :

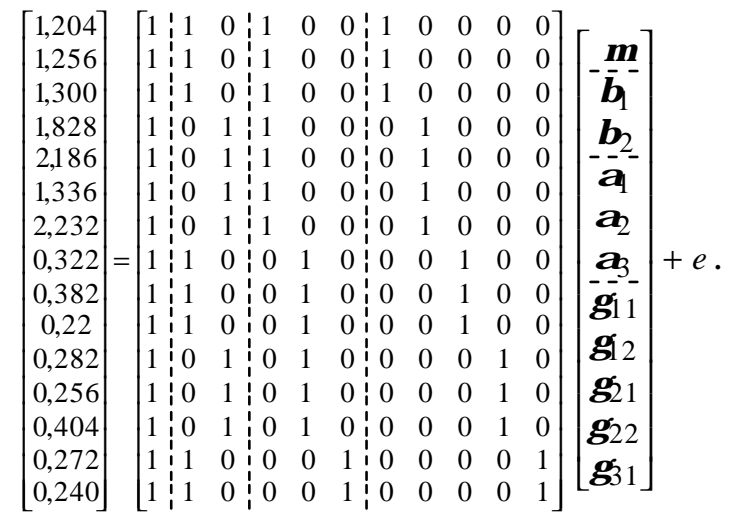

Construindo-se o sistema de equações normais com a restrição paramétrica, obteve-se para os dados em questão:

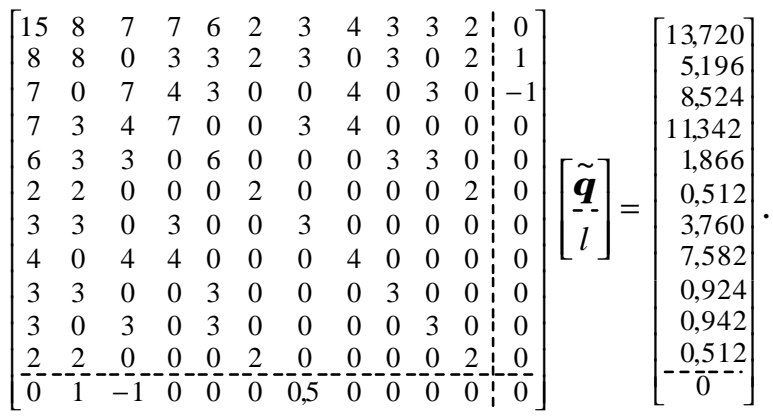




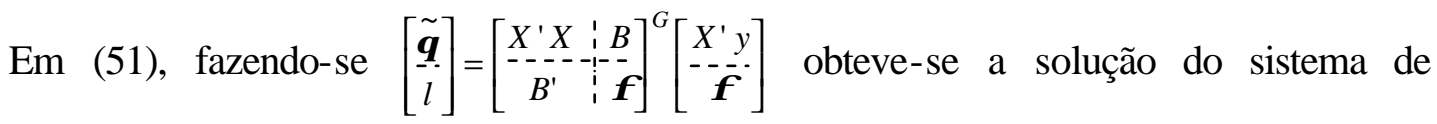
equações normais com a restrição paramétrica:

$$
\tilde{\boldsymbol{\Theta}}=\left[\begin{array}{r}
0,369 \\
0,184 \\
0,184 \\
0,680 \\
-0,162 \\
-0,149 \\
0,019 \\
0,661 \\
-0,084 \\
-0,078 \\
-0,149
\end{array}\right]
$$

$\mathrm{O}$ vetor $\tilde{y}$ é obtido como em (46). Calculando-se $\tilde{e}^{\prime} \tilde{e}=S Q_{\tilde{R} e s}$, tem-se que $S Q_{\tilde{\mathrm{R} e} s}=0,546$ é a soma de quadrados do resíduo para o modelo com a restrição paramétrica $B^{\prime} \boldsymbol{\theta}=\phi$.

Para o cálculo da soma de quadrados usual do resíduo, os vetores $\hat{y}$ e $\hat{e}$ são os mesmos obtidos em (47) e (48). Então, $\hat{e}^{\prime} \hat{e}=\|\hat{e}\|^{2}=S Q_{\operatorname{Re} s}=0,546$.

Por diferença verificou-se o acréscimo $(\boldsymbol{\delta})$ :

$$
\delta=S Q_{\tilde{\operatorname{Re}} s}-S Q_{\operatorname{Re} s}=0,546-0,546=0 .
$$

Esse $\delta=0$ é o acréscimo na $S Q_{\operatorname{Res}}$, motivado pela imposição da restrição paramétrica. Como $\delta=0$, a restrição paramétrica não é estimável e, em consequiência, a hipótese $H_{0}^{*-B}$ não é testável.

d) Hipóteses do tipo III para o Fator $B$

A hipótese $H_{0}^{3-B}$ no modelo-S (Tabela 27), tem como função paramétrica $B^{\prime} \boldsymbol{\theta}=\phi$, em que

$$
B^{\prime}=\left[\begin{array}{lllllllllll}
0 & 1 & -1 & 0 & 0 & 0 & 0,5 & -0,5 & 0,5 & -0,5 & 0
\end{array}\right]
$$

é a matriz com os coeficientes da função paramétrica.

O modelo linear e as equações na forma matricial são apresentados em (50). 
Como em (51), o sistema de equações normais com a restrição paramétrica obtido foi:

$\left[\begin{array}{ccccccccccc:c}15 & 8 & 7 & 7 & 6 & 2 & 3 & 4 & 3 & 3 & 2 & 0 \\ 8 & 8 & 0 & 3 & 3 & 2 & 3 & 0 & 3 & 0 & 2 & 1 \\ 7 & 0 & 7 & 4 & 3 & 0 & 0 & 4 & 0 & 3 & 0 & -1 \\ 7 & 3 & 4 & 7 & 0 & 0 & 3 & 4 & 0 & 0 & 0 & 0 \\ 6 & 3 & 3 & 0 & 6 & 0 & 0 & 0 & 3 & 3 & 0 & 0 \\ 2 & 2 & 0 & 0 & 0 & 2 & 0 & 0 & 0 & 0 & 2 & 0 \\ 3 & 3 & 0 & 3 & 0 & 0 & 3 & 0 & 0 & 0 & 0 & 0,5 \\ 4 & 0 & 4 & 4 & 0 & 0 & 0 & 4 & 0 & 0 & 0 & -0,5 \\ 3 & 3 & 0 & 0 & 3 & 0 & 0 & 0 & 3 & 0 & 0 & 0,5 \\ 3 & 0 & 3 & 0 & 3 & 0 & 0 & 0 & 0 & 3 & 0 & -0,5 \\ 2 & 2 & 0 & 0 & 0 & 2 & 0 & 0 & 0 & 0 & 2 & 0 \\ \hdashline 0 & 1 & -1 & 0 & 0 & 0 & 0,5 & -0,5 & 0,5 & -0,5^{-0} & 0\end{array}\right]\left[\begin{array}{c}\boldsymbol{\Theta} \\ \hdashline \\ l\end{array}\right]=\left[\begin{array}{r}13,720 \\ 5,196 \\ 8,524 \\ 1,342 \\ 1,866 \\ 0,512 \\ 3,760 \\ 7,582 \\ 0,924 \\ 0,942 \\ 0,512 \\ \hdashline 0\end{array}\right]$.

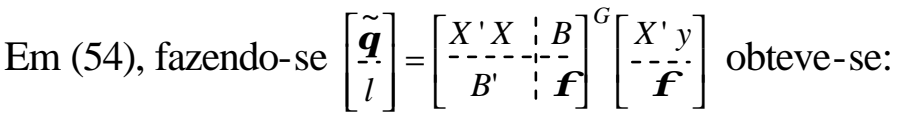

$$
\tilde{\boldsymbol{\Theta}}=\left[\begin{array}{r}
0,376 \\
0,164 \\
0,212 \\
0,688 \\
-0,169 \\
-0,142 \\
0,198 \\
0,489 \\
0,109 \\
-0,278 \\
-0,142
\end{array}\right] .
$$

em que $\tilde{\Theta}$ é a solução do sistema de equações normais com a restrição paramétrica.

Com $\tilde{\boldsymbol{\theta}}$ calculou-se a aproximação para o vetor $y$ de observações $(\tilde{y}=X \tilde{\boldsymbol{\theta}})$. Assim, obteve-se $\tilde{e}=y-\tilde{y}$ e então $\tilde{e}^{\prime} \tilde{e}=S Q_{\tilde{R} e s}$. Nesse caso, $S Q_{\tilde{R} e s}=0,882$ é a soma de quadrados do resíduo para o modelo com a restrição paramétrica $B^{\prime} \boldsymbol{\theta}=\boldsymbol{\phi}$.

$\mathrm{O}$ vetor $\hat{y}$ (aproximação de mínimos quadrados para o vetor $y$ das observações) e o vetor $\hat{e}$ (erro de ajustamento) são os mesmos obtidos em (42) e (43) respectivamente, para o cálculo de $S Q_{H_{0}^{3-A}}$.

Dessa forma, tem-se a soma de quadrados usual do resíduo:

$$
\hat{e}^{\prime} \hat{e}=\|\hat{e}\|^{2}=S Q_{\operatorname{Re} s}=0,546 .
$$

Verificando-se o acréscimo $(\boldsymbol{\delta})$ na soma de quadrados usual do resíduo: 


$$
\delta=S Q_{\tilde{R} e s}-S Q_{\operatorname{Re} s}=0,882-0,546=0,336 .
$$

Esse valor de $\delta$ é o acréscimo verificado na $S Q_{\operatorname{Res}}$ motivado pela imposição da restrição paramétrica.

Como $\delta>0$, conclui-se que a restrição paramétrica é linearmente dependente, ou seja, estimável, e portanto a hipótese é testável. Assim, $\delta$ corresponde à soma de quadrados associada à hipótese $H_{0}^{3-B}$, ou seja, $S Q H_{0}^{3-B}=0,336$.

Fazendo-se:

$$
F_{\text {Obs }}=\frac{\frac{0,336}{1}}{0,0546}=6,15,
$$

obtém-se o valor da estatística $F$ para a hipótese $H_{0}^{3-B}$, que está de acordo com o apresentado na Tabela 28 e com o obtido utilizando a função CONTRAST do PROC GLM. A interpretação do resultado é análoga ao que já foi apresentado.

Nota-se portanto que o uso da técnica da restrição paramétrica, permite uma forma alternativa para teste de qualquer hipótese de interesse do pesquisador. Assim, é possível elaborar e testar quaisquer hipóteses que se deseje. Evidentemente, caso a hipótese não seja testável, o valor de $\delta$ será zero.

Tendo em vista os numerosos cálculos envolvidos na técnica da restrição paramétrica, foi elaborado um programa interativo entre o procedimento GLM e o módulo IML (Interactive Matrix Language) do SAS (ver APÊNDICE). O programa é elaborado de forma que, para uma hipótese testável, será realizada a análise de variância com o emprego da técnica da restrição paramétrica. Para funções paramétricas não estimáveis, o usuário será informado de que sua hipótese não é testável, e pode então optar por construir outras hipóteses.

Como pode ser visto nas situações desenvolvidas em 4.3.2 e 4.3.3, a técnica da restrição paramétrica e a função CONTRAST do PROC GLM são equivalentes no teste de hipóteses, obtendo os mesmos resultados. Assim, quando o pesquisador desejar 
formular as hipóteses do seu interesse poderá testá-las por qualquer uma das técnicas discutidas.

\subsection{Considerações gerais}

Frente às situações discutidas nesse texto, percebe-se que o sistema estatístico SAS nem sempre fornece automaticamente o teste para hipóteses de interesse imediato. Em muitas situações, há interesse em desdobrar graus de liberdade de tratamentos. No conjunto A, por exemplo, o interesse é obter informações com relação à comparação entre as variedades dentro de cada grupo. Para o conjunto B, deseja-se verificar quais híbridos diferem entre si, também dentro de cada grupo. Nessas situações, o PROC GLM do SAS oferece como opção a construção de contrastes e o teste por meio da função CONTRAST. Dessa forma, testa-se qualquer hipótese formulada pelo pesquisador, sem preocupação com a estimabilidade, pois caso a função paramétrica não seja estimável, o SAS informa o usuário quanto a esse fato. Entretanto, quando o número de elementos dentro de cada grupo é elevado, torna-se trabalhoso construir os contrastes. A construção de contrastes entre os grupos é de fácil construção, pois no geral têm-se no máximo 3 ou 4 grupos.

Diante do que foi exposto, esse trabalho propõe o uso de variáveis auxiliares como uma forma simples e elegante para minimizar esses problemas, principalmente para determinar diferenças dentro dos grupos. Embora por meio de variáveis auxiliares não seja possível determinar diferenças entre os grupos, sendo necessário utilizar a função CONTRAST, isso é facilmente implementado em situações práticas, uma vez que a maior dificuldade é construir os contrastes dentro de cada grupo, e não entre os grupos.

Outra situação abordada é em modelos de classificação dupla cruzada e em presença de caselas vazias. Nesse caso, o SAS fornece como opções os quatro tipos de hipóteses, que geralmente não são de fácil interpretação. Além disso, o usuário pode ter interesse em hipóteses específicas. $\mathrm{O}$ uso de restrições paramétricas estimáveis e de contrastes pode ajudar, no mínimo, para verificar se é possível testar o que se espera. Em caso contrário, será um incentivo para que o pesquisador estude com cautela um pouco mais a situação encontrada. 


\section{CONCLUSÕES}

Diante do exposto nesse trabalho, concluiu-se que:

i) O uso da técnica de variáveis auxiliares facilita o desdobramento ortogonal dos graus de liberdade de tratamentos para a comparação dos tratamentos dentro de grupos pré-estabelecidos;

ii) Essa técnica tem resultados equivalentes aos obtidos utilizando-se a função CONTRAST do PROC GLM do SAS;

iii) O uso de restrições paramétricas estimáveis permite ao pesquisador testar diretamente hipóteses de seu interesse. Dessa forma pode ser visto como um critério alternativo para realizar testes de hipóteses em modelos lineares com dados desbalanceados;

iv) A função CONTRAST do PROC GLM do SAS e o critério baseado no emprego de restrição paramétrica estimável são equivalentes quanto à realização de testes de hipóteses de interesse em experimentos desbalanceados. 


\section{REFERÊNCIAS BIBLIOGRÁFICAS}

BANZATTO, D.A.; KRONKA, S. N. Experimentação agrícola. 3.ed. Jaboticabal: FUNEP, 1995. 245p.

CAMARINHA FILHO, J.A. Testes de hipóteses em modelos lineares com dados desbalanceados e caselas vazias. Piracicaba, 1995. 142p. Dissertação (Mestrado) - Escola Superior de Agricultura “Luiz de Queiroz”, Universidade de São Paulo.

CAMPOS, H. de. Estatística aplicada à experimentação com cana-de-açúcar. Piracicaba: FEALQ, 1984. 292p.

ELSTON, R.C.; BUSH, J. The hypotheses that can be tested when there are interactions in an analysis of variance model. Biometrics, v.20, n.1, p.681-699, 1964.

GRAYBILL, F. A. An introduction to linear statistical models. New York: McGrawHill, 1961. 461p. 
HENDERSON, C.R. Estimation of variance and covariance components. Biometrics, v.9, p.226-252, 1953.

HOCKING, R.R.; SPEED, F.M. A full rank analysis of some linear model problems. Journal of the American Statistical Association, v.70, p.706-712, 1975.

HOCKING, R.R. The analysis of linear models. Monterrey: Brooks/Cole, 1985. $385 p$.

IEMMA, A.F. Modelos lineares: uma introdução para profissionais da pesquisa agropecuária. Piracicaba: FEALQ, 1985. 145p.

IEMMA, A.F. Modelos lineares: uma introdução para profissionais da pesquisa agropecuária. 2.ed. Londrina: Imprensa Oficial do Estado do Paraná, 1987. 263p.

IEMMA, A.F. Matrizes para estatística. Departamento de ciências exatas. Piracicaba: ESALQ, 1989. 418p.

IEMMA, A.F. Testes de hipóteses em modelos lineares com amostras desequilibradas. Gembloux: $\varphi \sigma \rho$ publicações, 1991. 101p. 
IEMMA, A.F. Análisis de varianza de experimentos con celdas vácias. Córdoba: $\varphi$ $\sigma \rho$ publicações, 1993. 102p.

IEMMA, A.F. Que hipóteses estatísticas testamos através do SAS em presença de caselas vazias? Scientia Agricola, v.52, n.2, p. 210-220, 1995.

IEMMA, A.F.; PERRI, S.H.V. Ajuste de modelos fixos desbalanceados através do sistema estatístico SAS. Piracicaba: ESALQ, Departamento de Matemática e Estatística, 1997. 99p.

MONDARDO, M. Estimabilidade de funções paramétricas com dados desbalanceados através do PROC GLM do SAS: Aplicações à pesquisa agropecuária. Piracicaba, 1994. 166p. Dissertação (Mestrado) - Escola Superior de Agricultura "Luiz de Queiroz", Universidade de São Paulo.

MONDARDO, M.; IEMMA, A. F. Considerações sobre as funções estimáveis fornecidas pelo PROC SAS/GLM para dados desbalanceados. Scientia Agricola, v.55, n.2, p.172-182, 1998.

NESI, A. N. Multiplicação e alongamento in vitro de microestacas de porta-enxertos de macieira (Malus domestica Borkh). Pelotas, 2000. 63p. Dissertação (Mestrado) Faculdade de Agronomia “Eliseu Maciel”, Universidade Federal de Pelotas. 
NESI, C.N.; IEMMA, A.F. Restrições paramétricas e testes de hipóteses sobre dados desbalanceados. In: REUNIÃO ANUAL DA REGIÃO BRASILEIRA DA SOCIEDADE INTERNACIONAL DE BIOMETRIA, 46.; SIMPÓSIO DE ESTATÍSTICA APLICADA À EXPERIMENTAÇÃO AGRONÔMICA， 9., Piracicaba, 2001. Anais. Piracicaba: FEALQ, 2001. p.619-622.

OVERALL, J.E.; SPIEGEL, D.K. Concerning least squares analysis of experimental data. Psychological Bulletin, v.72, p.311-322, 1969.

PERECIN, D.; MALHEIROS, E. B.; PEREIRA, G.T. Variáveis auxiliares para expressar desdobramentos de graus de liberdade e contrastes com o programa SAS. In: REUNIÃO ANUAL DA REGIÃO BRASILEIRA DA SOCIEDADE INTERNACIONAL DE BIOMETRIA, 45.; São Carlos, 2000. Anais. São Carlos: ABE, 2000. p.137-140.

PIMENTEL GOMES, F. Curso de estatística experimental. 13.ed. Piracicaba: Nobel, 1990. $467 p$.

RAO, C. R. On the linear combination of observations and the general theory of least squares. Sankhyã: The Indian Journal of Statistics, v.7, n.3, p.237-256, 1945. 
SANTOS, E. S. Utilização de "softwares" estatísticos na interpretação de hipóteses com dados desbalanceados. Piracicaba, 1994. 175p. Tese (Doutorado) - Escola Superior de Agricultura “Luiz de Queiroz”, Universidade de São Paulo.

SAS INSTITUTE. SAS/STAT user's guide: version 6. 4.ed. Cary, 1989. v.2, 846p.

SCHEFFÉ, H. The analysis of variance. 2.ed. New York: John Wiley, 1961. 477p.

SEARLE, S. R. Linear models. New York: John Wiley, 1971. 532p.

SEARLE, S. R. Linear models for unbalanced data. New York: John Wiley, 1987. 536 .

SCOTT, R.A.; MILLIKEN, G.A. A SAS program for analysing augmented randomized complete block design. Crop Science, v.33, p.865-867, 1993.

SPEED, F.M.; HOCKING, R.R.; HACKNEY, O.P. Methods of analysis of linear models with unbalanced data. Journal of the American Statistical Association, v. 73, p.105-112, 1978. 
YATES, F. The analysis of multiple classifications with unequal numbers in the different classes. Journal of the American Statistical Association, v.29, p.51-56, 1934. 
APÊNDICE 


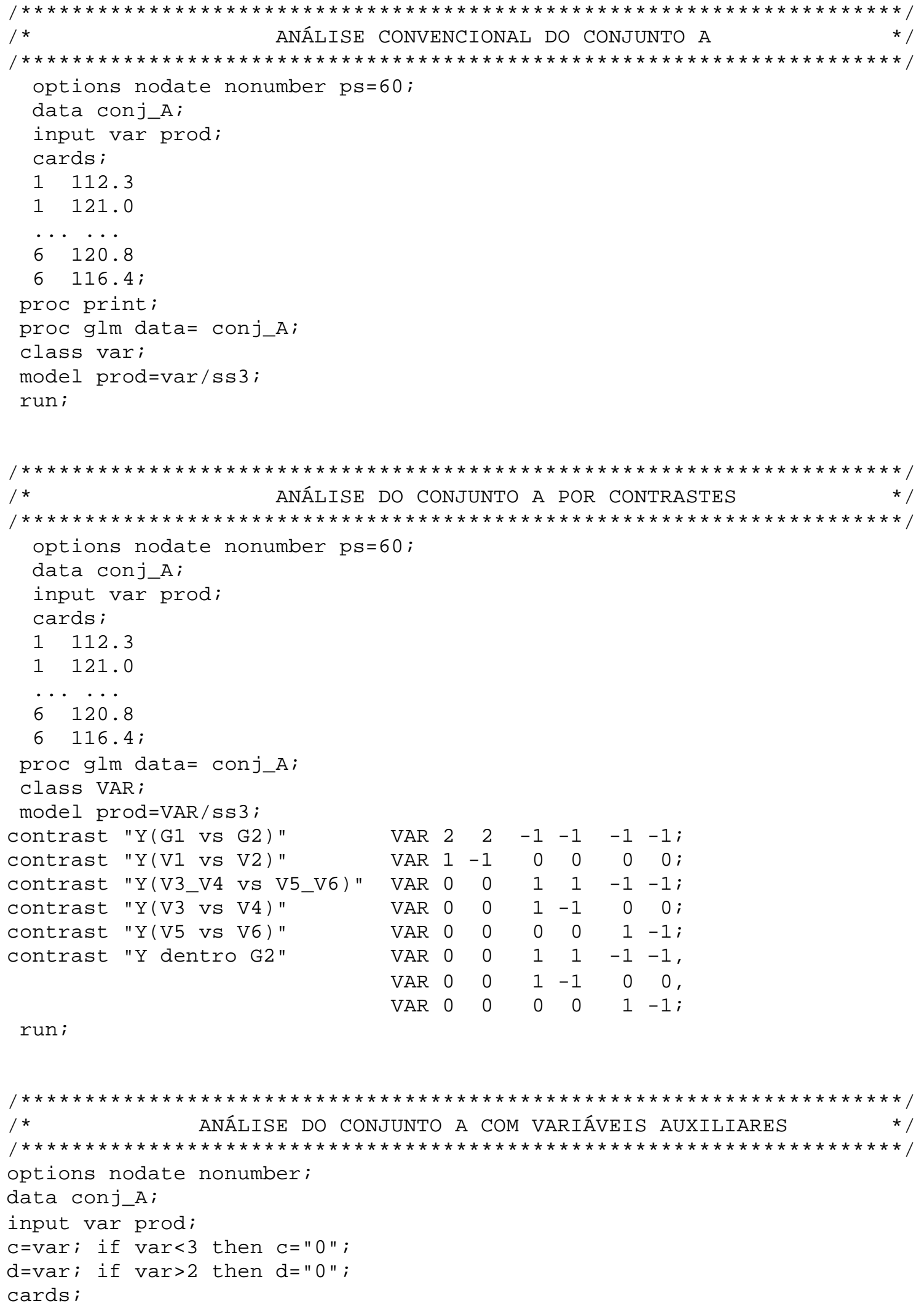

run; 


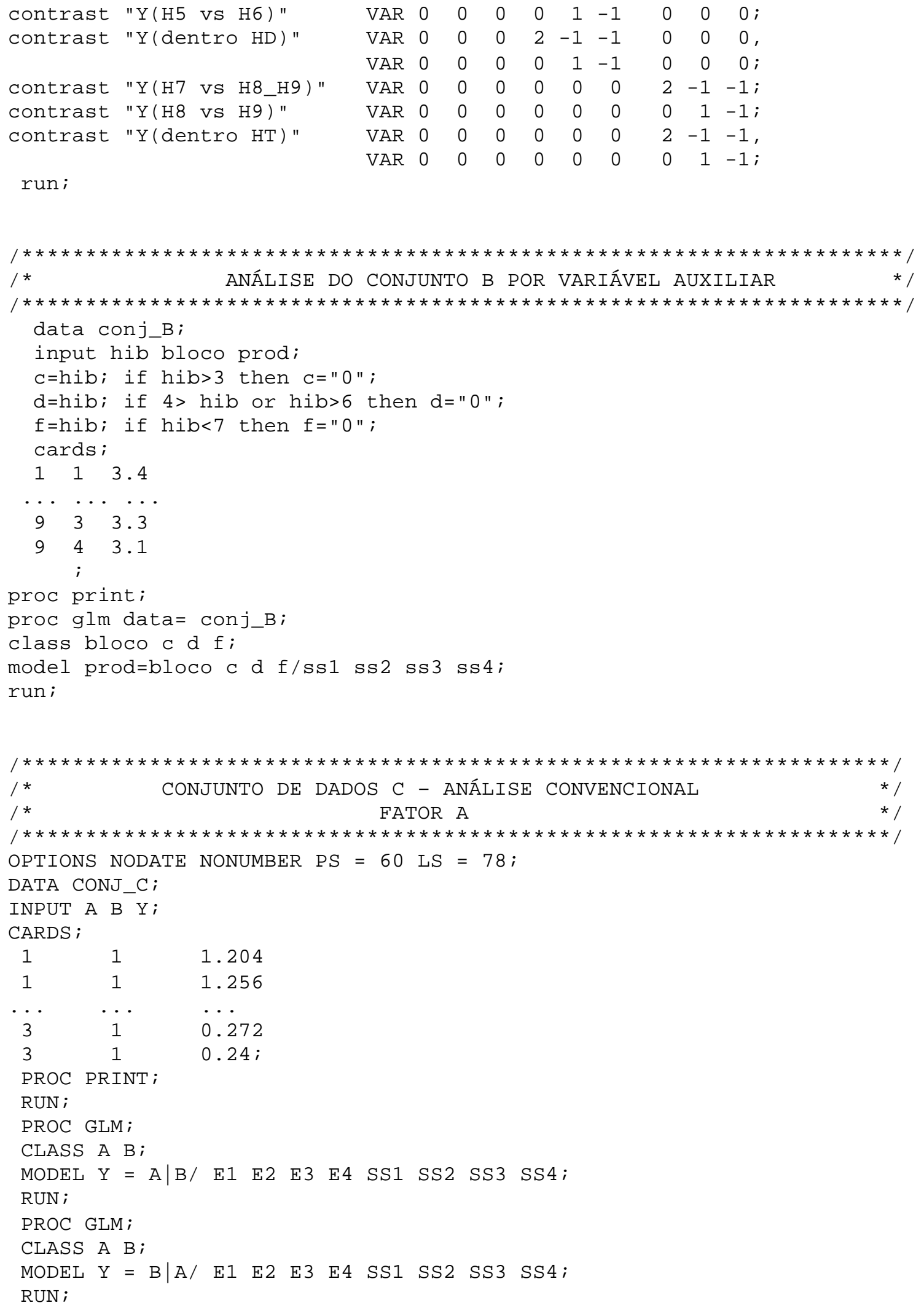




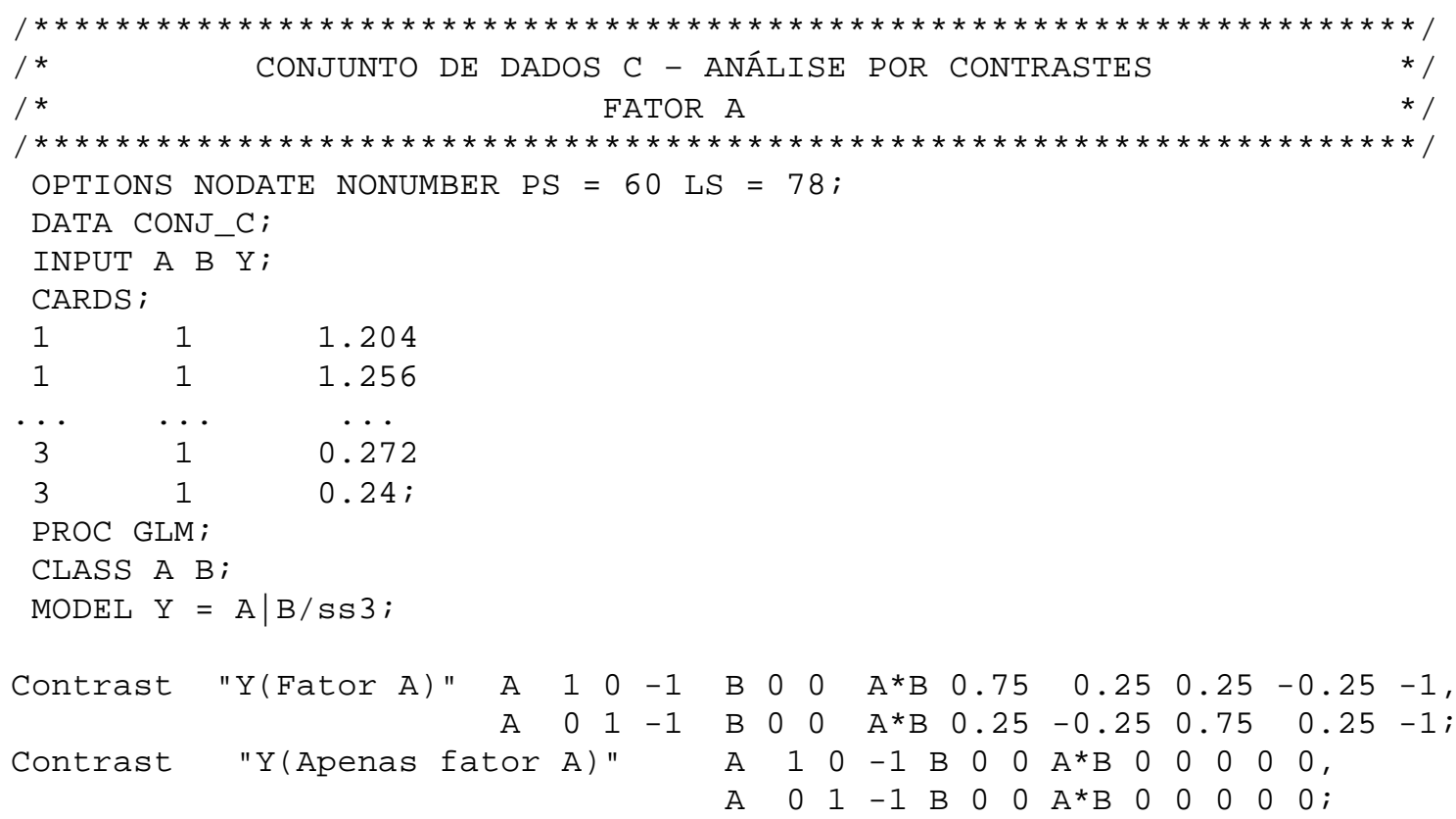

RUN;

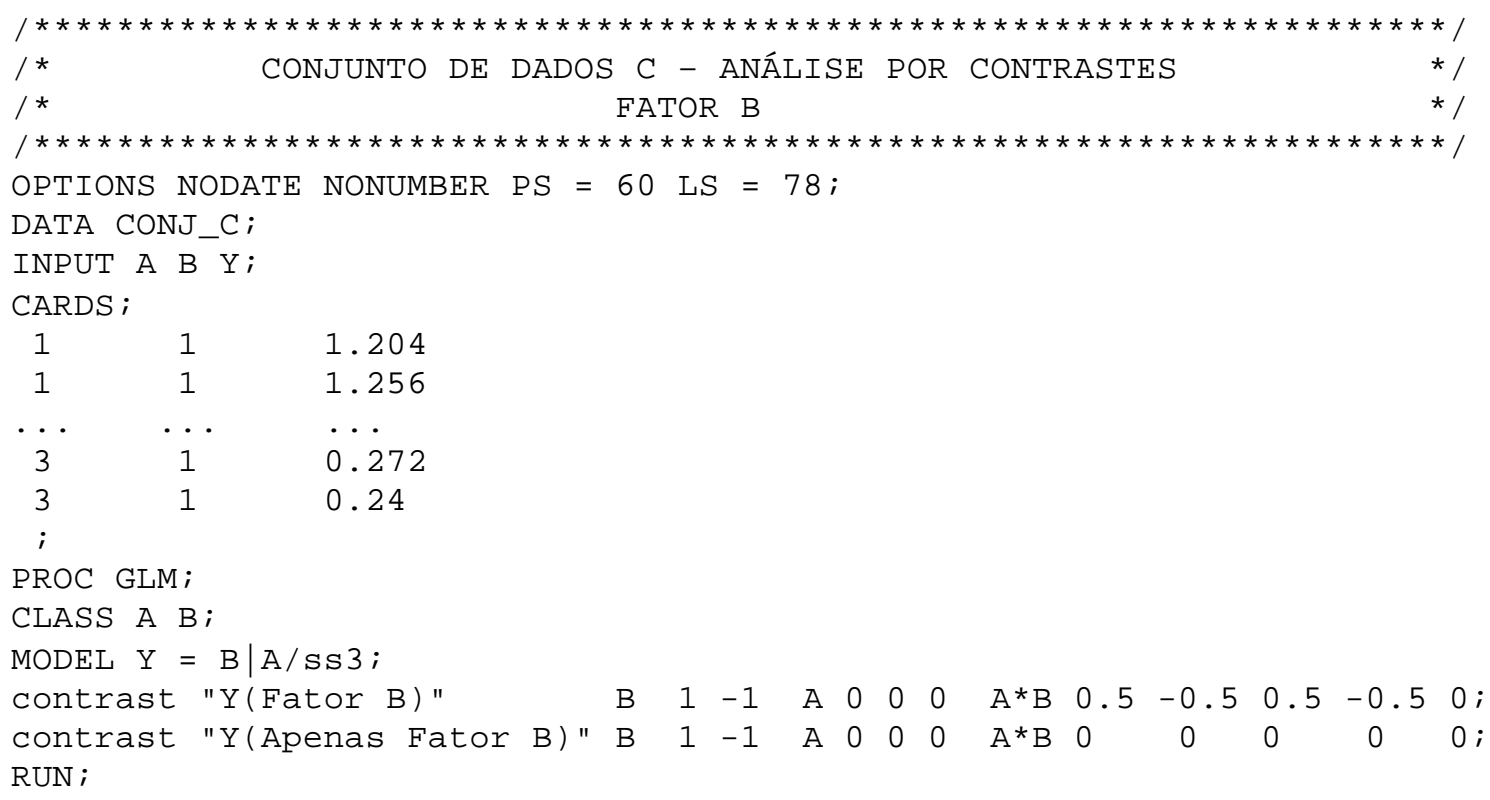




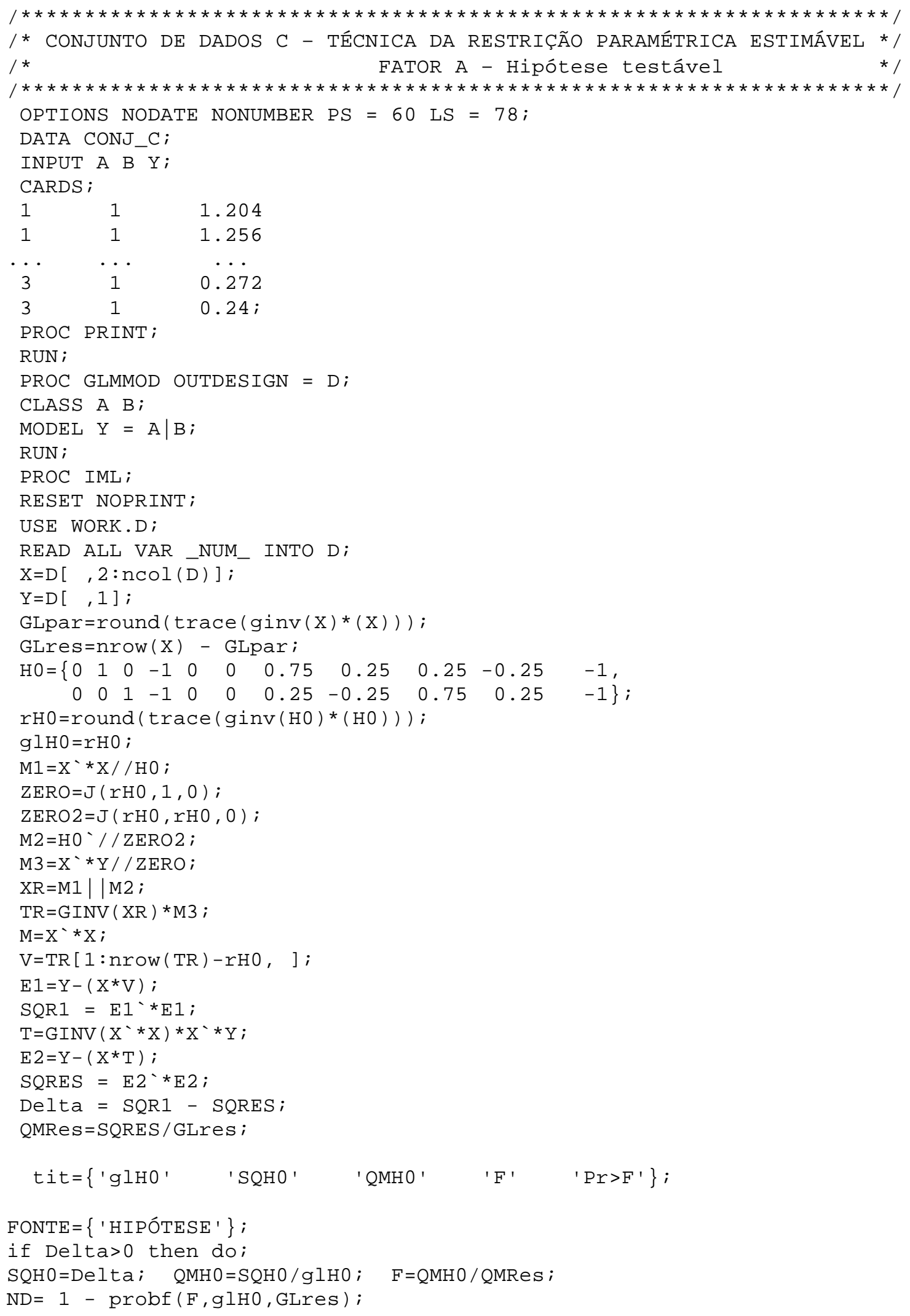




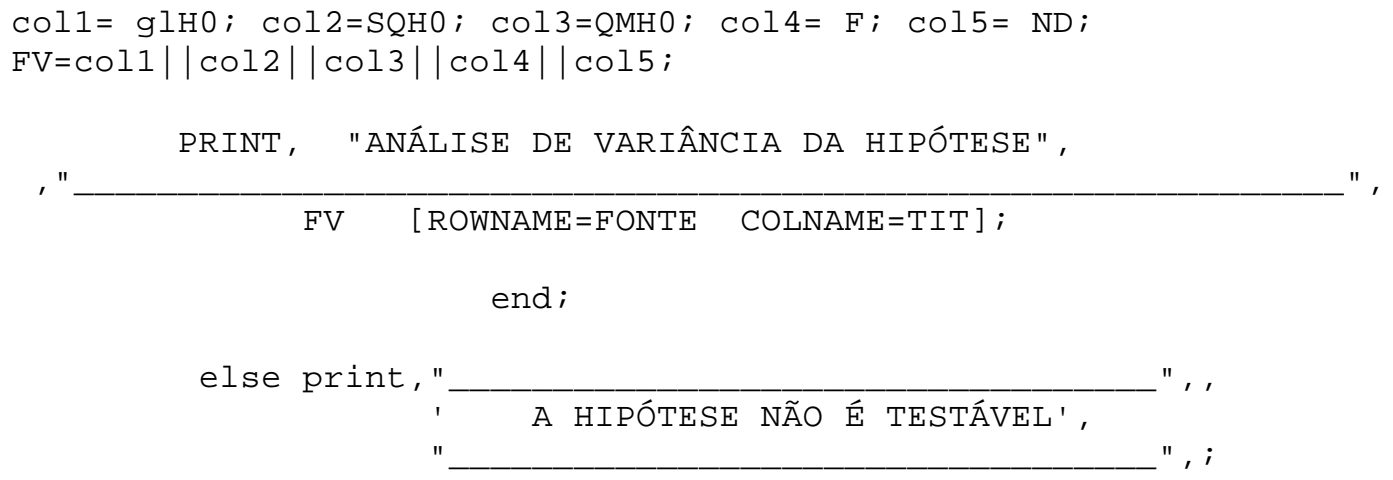

QUIT;

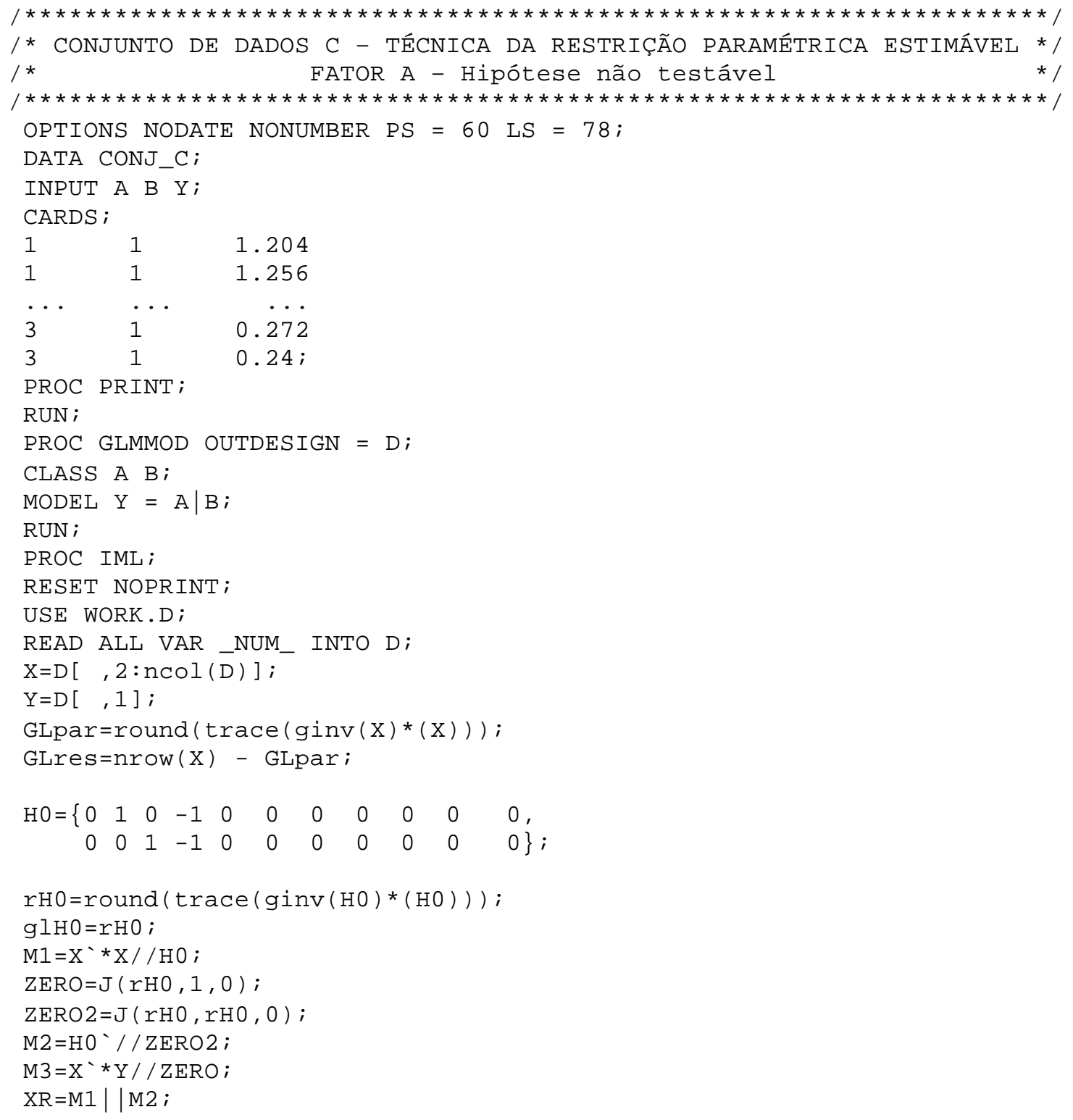




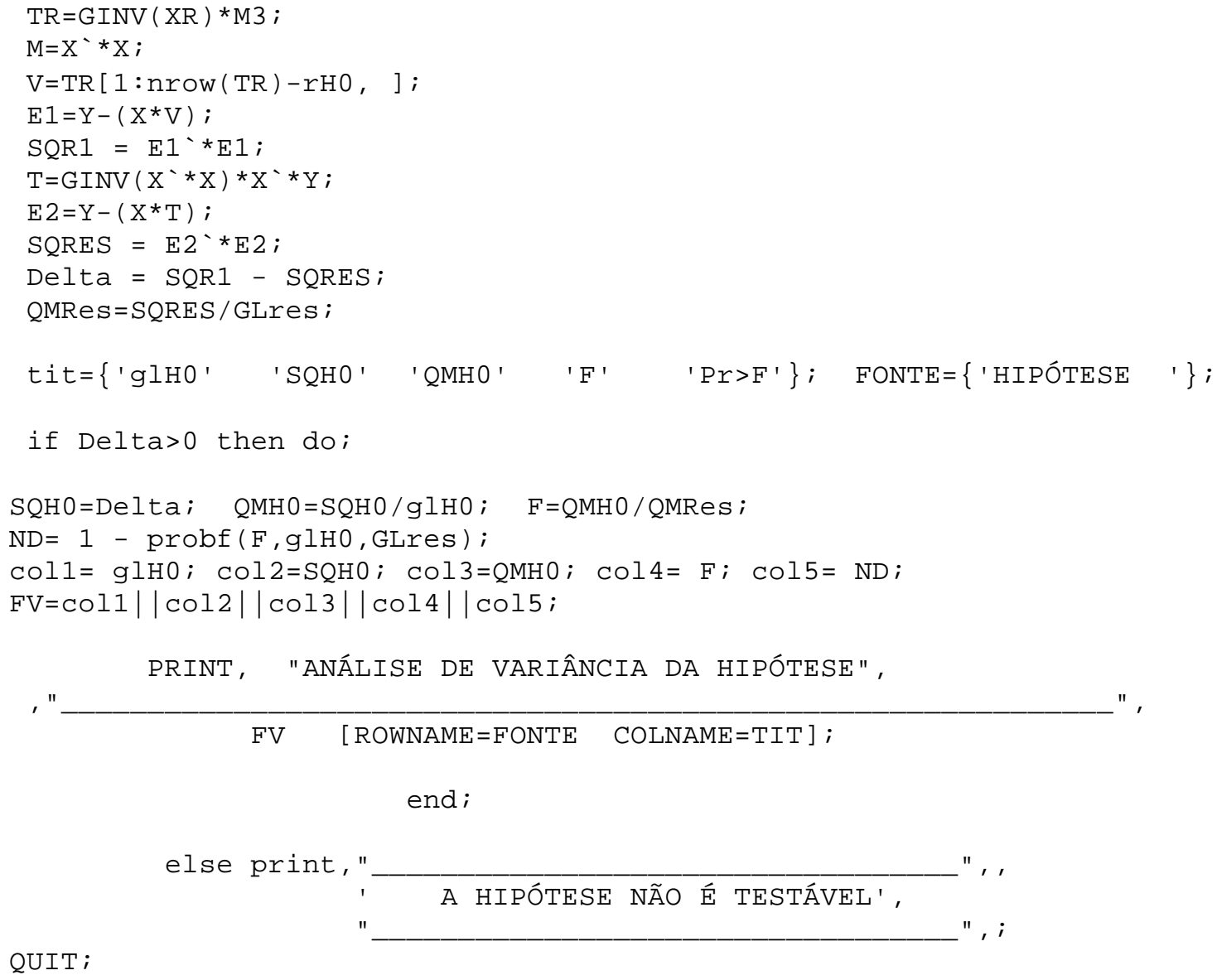




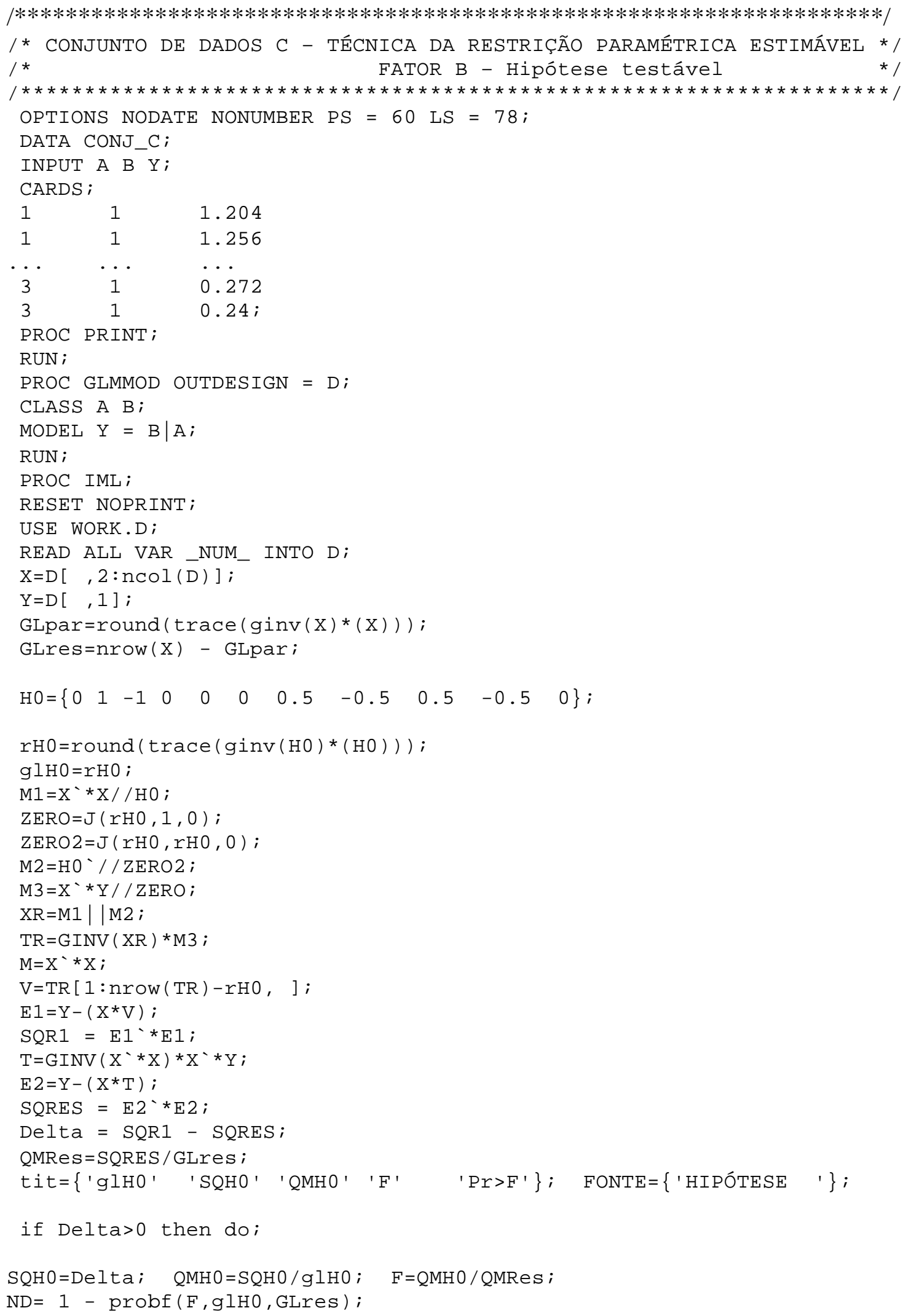




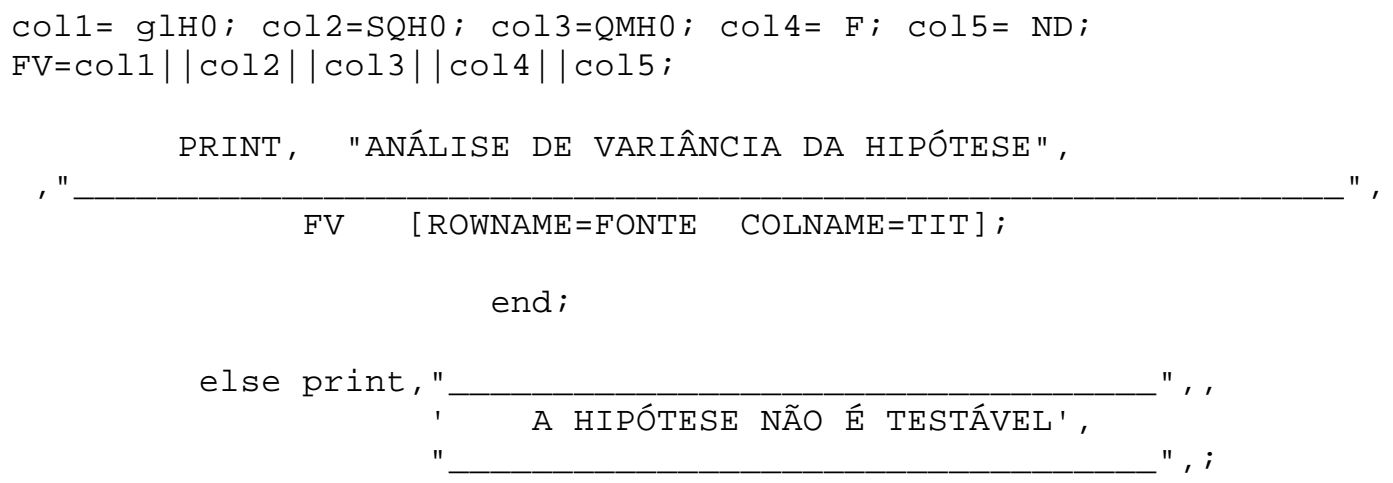

QUIT;

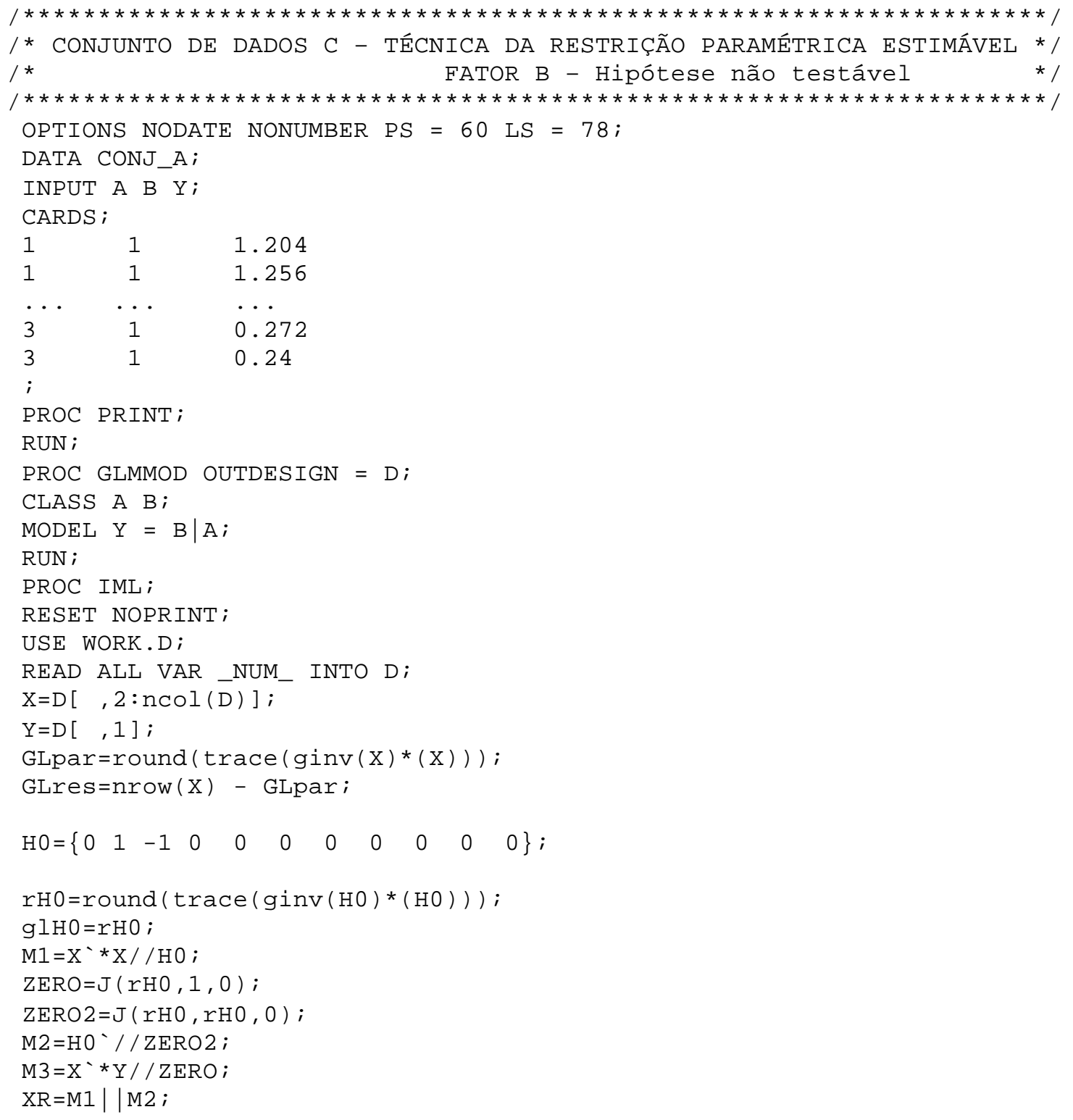




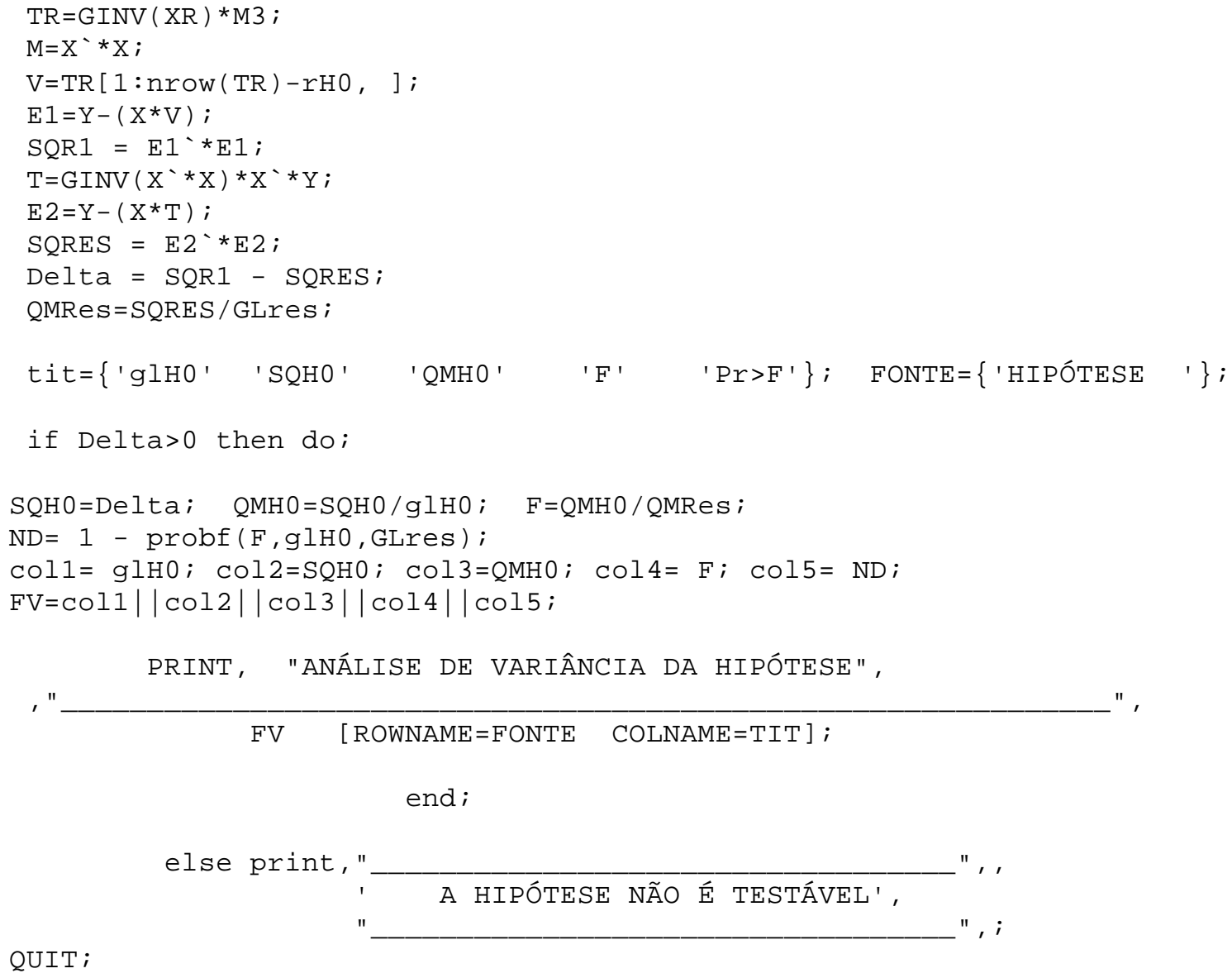

